\title{
HOT LANE POLICIES AND THEIR IMPLICATIONS
}

\author{
A Thesis \\ by \\ RAHUL GOEL \\ Submitted to the Office of Graduate Studies of \\ Texas A\&M University \\ in partial fulfillment of the requirements for the degree of \\ MASTER OF SCIENCE
}

May 2010

Major Subject: Civil Engineering 


\title{
HOT LANE POLICIES AND THEIR IMPLICATIONS
}

\author{
A Thesis \\ by \\ RAHUL GOEL \\ Submitted to the Office of Graduate Studies of \\ Texas A\&M University \\ in partial fulfillment of the requirements for the degree of \\ MASTER OF SCIENCE
}

\begin{abstract}
Approved by:
Chair of Committee, Mark Burris

Committee Members, Katherine Turnbull

Bruce Wang

Head of Department, John Niedzwecki
\end{abstract}

May 2010

Major Subject: Civil Engineering 


\author{
ABSTRACT \\ HOT Lane Policies and Their Implications. (May 2010) \\ Rahul Goel, B.Tech., Indian Institute of Technology, Delhi, India \\ Chair of Advisory Committee: Dr. Mark Burris
}

High-Occupancy toll (HOT) lanes allow lower-occupant vehicles (LOVs) to use a HOV lane for a fee, while maintaining free travel to qualifying HOVs. HOT lanes are gaining interest throughout the country as a strategy for meeting multiple performance objectives in congested urban freeway corridors. Currently there are ten fully operational HOT lanes around the country in seven different states and this research examined the nine of them (excluding I-35 W). Even with only a handful of operational HOT lane projects, there is great diversity in terms of HOT lane design and operations. With HOT lane implementation there are many issues, including: toll rates, vehicle occupancy requirement, number of access points, and safety.

This research examined (i) the different factors which lead to the development of the HOT lanes in their respective corridors (ii) the objectives of the HOT lanes (iii) changes made in the corridor due to HOT lane implementation (iv) the different impacts of the HOT lanes and (v) the extent to which the objectives of the HOT lanes were achieved. Using three pairs of HOT lanes with similar design and operational characteristics, comparisons were made to examine the impacts of the similar HOT lanes in two different corridors.

With the strict registration requirement for HOV3+ on the I-95 Express Lanes there were indications that some carpoolers broke up in to lower occupancy vehicles. Tolled access for HOV2s on I-95 as well as the SR 91 Express Lanes resulted in lower usage of the Express Lanes by the HOV2s (fewer than 30 percent of the total corridor HOV2s) as compared to a conventional HOV lane (60 percent) where HOV2 access is free. The effect of availability of transit on the HOT lanes can also be seen from SR 91 as compared to I-95. On SR 91, the Express bus does not use the Express Lanes and there was almost no change in its ridership after the Express Lanes were implemented. However, on I-95, the Express bus uses the Express Lanes 
and travel time of buses decreased by 17 minutes due to Express Lanes implementation. The Express bus ridership also increased by 30 percent.

On the SR167 and I-25 HOT lanes, the exogenous factors like gas prices and economic recession seemed to influence the usage of the HOT lanes. In both the HOT Lanes, carpool usage was positively correlated to the gasoline prices. On I-25, the increasing unemployment rate coincided with the decreasing toll paying travelers. On SR 167 there were also indications of mode shifts among the transit, carpool and toll paying SOVs due to fluctuating gas prices. With declining gas prices, the transit and carpool usage went down while toll paying users increased.

An inverse relationship between the convenience of access points and the safety perceived by the HOT lane users was found. For example, I-15 Express Lanes in Salt Lake City reduced the access points from unrestricted with the previous HOV lanes to limited with the Express Lanes. As a result, more predictable merging led to an increase in the perceived safety of the Express lanes as well as the speed of the corridor. On the other hand, some carpoolers mentioned not using the Express Lanes anymore because of access inconvenience. The access inconvenience was also mentioned by previous carpoolers in HOV lanes on I-95 as one of the reasons for not using the Express Lanes. These findings underscore the importance of outreach programs during the planning process of the HOT lanes to minimize the confusion among the previous users of the HOV lanes and spreading awareness among them regarding the increased safety benefits. 


\section{DEDICATION}

I would like to dedicate this thesis to God almighty whose presence is always felt in my heart. I also dedicate it to my parents for their unflinching support and love for me. I am also thankful to the Indian Institute of Technology, Delhi for giving me one of the best learning environments in the country and without which I would not be what I am today. I am also thankful to a dear friend of mine- Nitish Umang who was always there to show me a ray of hope whenever I needed it most. 


\section{ACKNOWLEDGEMENTS}

I would like to thank my advisor, Dr. Mark Burris for the guidance and support throughout the course of this research. I would also like to thank my committee members, Dr. Katherine Turnbull and Dr. Bruce Wang for their valuable suggestions.

Thanks also go to my friends and colleagues and the department faculty and staff for making my time at Texas A\&M University a great experience. This research work uses the data collected during the Texas Transportation Institute (TTI) Project entitled "Factors Influencing HOT Lane Usage" funded by Federal Highway Administration (FHWA). 


\section{TABLE OF CONTENTS}

Page

ABSTRACT iii

DEDICATION. ..v

ACKNOWLEDGEMENTS................................................................................. vi

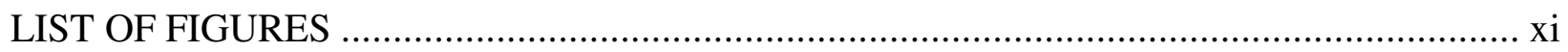

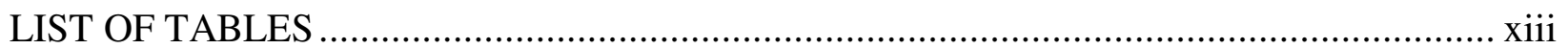

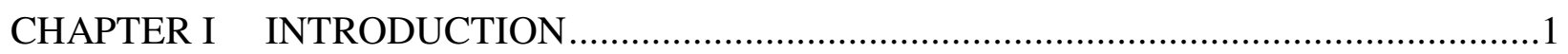

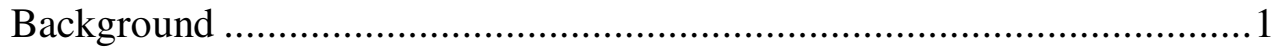

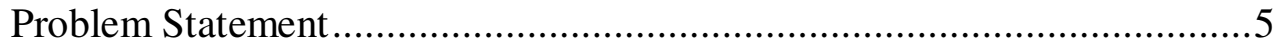

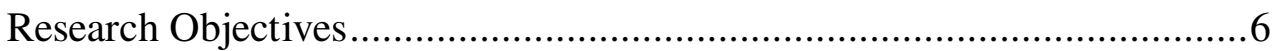

Research Methodology .............................................................

Thesis Organization ................................................................... 8

CHAPTER II ～LITERATURE REVIEW ............................................................

CHAPTER III SR 91 EXPRESS LANES, LOS ANGELES AND I-95 EXPRESS LANES,

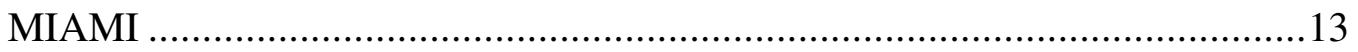

SR 91 Express Lanes: Introduction ..............................................13

Why Were HOT Lanes Considered?.................................................. 14

Policy of 3+ Vehicle Occupancy Requirement.................................... 15

Impact on Transit ............................................................... 15

Changes in Bus Operation after the Express Lanes Opened ................16

Rail and Bus Ridership ............................................................ 16

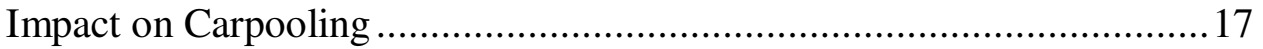

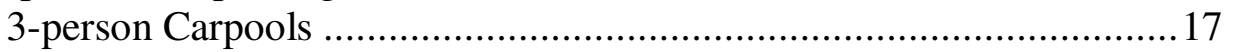

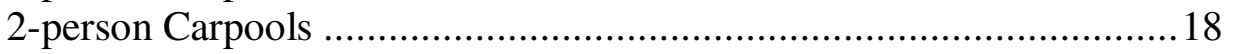

Safety Implications of the Express Lanes........................................ 19

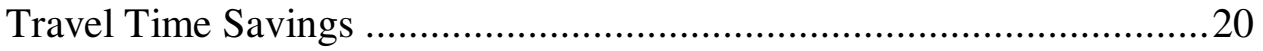

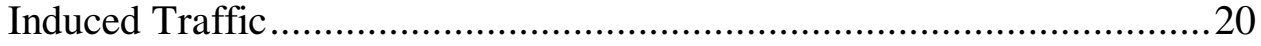

I-95 Express Lanes, Miami: Introduction..............................................21

Previous HOV Lane Operations .........................................................22

Why Were Express Lanes Considered? .............................................23

Changes in the Corridor Due to the Express Lanes ...............................24 


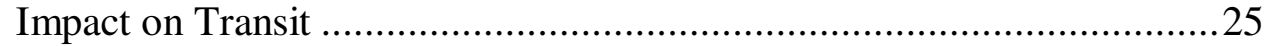

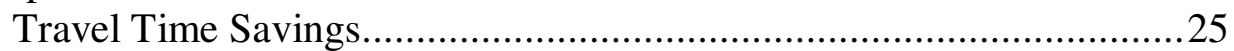

Ridership ..............................................................................26

Effect of Gas Prices ..................................................................... 27

Mode Shift Due to Transit ...........................................................22

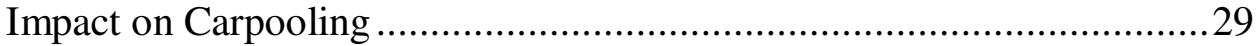

Findings from South Florida Region Commuter Survey (May 2009) ......29

Express Lane Users .....................................................................29

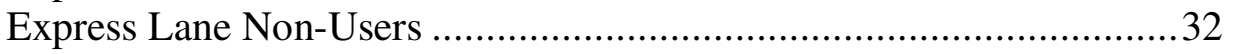

Safety Implications of the Express Lanes......................................... 34

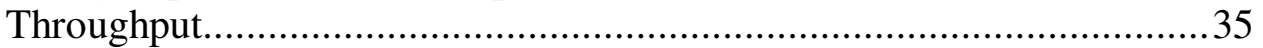

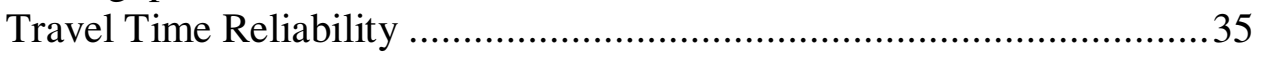

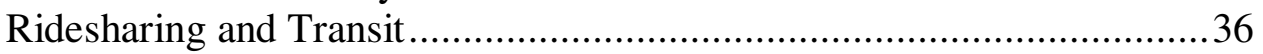

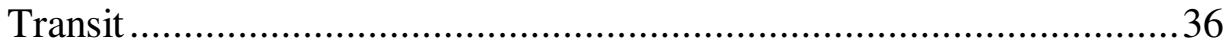

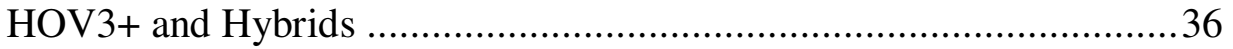

SR 91 Express Lanes and I-95 Express Lanes....................................37

Similarities between the Express Lanes .........................................3 37

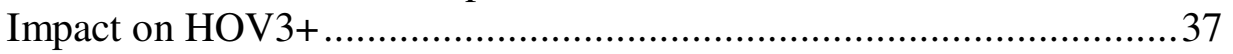

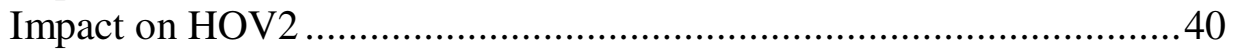

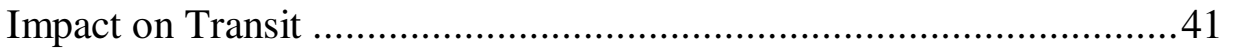

Impact on Safety.......................................................... 41

Usage of Express Lanes......................................................... 42

CHAPTER IV I-15 (FROM 1996 UNTIL 2008), SAN DIEGO AND I-25 EXPRESS LANES,

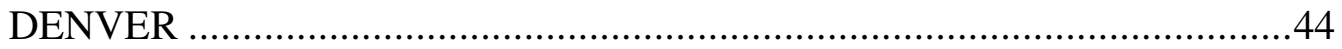

I-15 Express Lanes: Introduction .....................................................44

Timeline of the I-15 San Diego Express Lanes ...................................46

Previous HOV Lanes.....................................................................46

Traffic Conditions on the Corridor Before the Express Lanes ...............47

Peak Hour Volume ........................................................................ 47

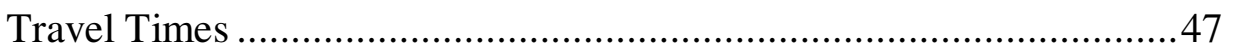

Why Were the Express Lanes Considered?.......................................4 47

Impact on Transit .........................................................................48

Impact on Carpooling .................................................................49

Usage of the Express Lanes ......................................................51

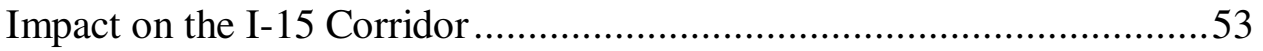

Safety Implications of the Express Lanes......................................53

Revenue for Transit ..................................................................53

I-25 Express Lanes, Denver: Introduction............................................54

Why Were Express Lanes Considered? .........................................55

How Policies Were Designed Based on Objectives................................57

Setting the Minimum Toll .......................................................57 


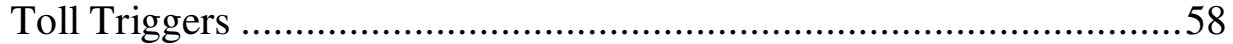

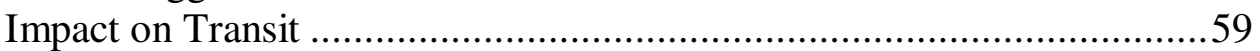

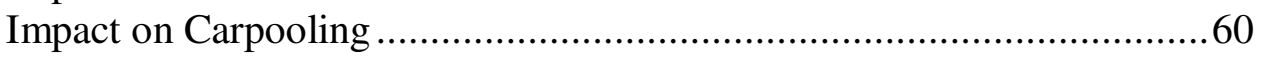

Trend of Express Lanes Usage ………………………......................61

I-15, San Diego Express Lanes and I-25 Express Lanes...........................63

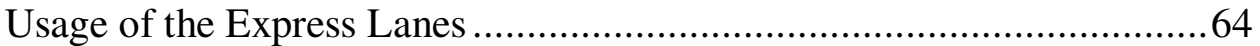

Safety Issues with the Express Lanes......................................................65

CHAPTER V I-394 EXPRESS LANES, MINNEAPOLIS AND SR 167 HOT LANES,

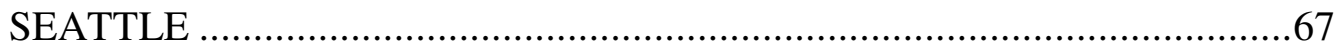

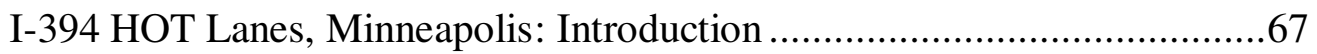

Previous HOV Lanes Operations ........................................................67

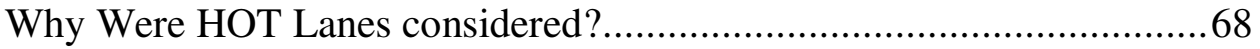

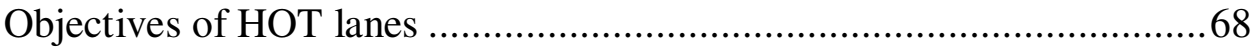

Important Issues during HOV to HOT Conversion ...................................71

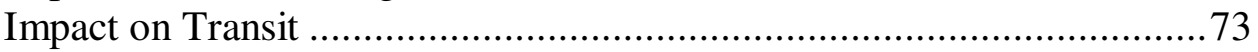

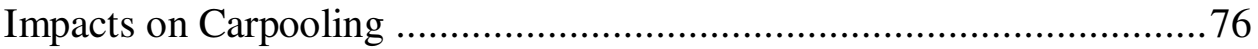

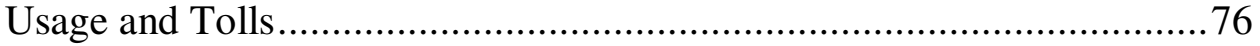

Vehicle and Person throughput.............................................................77

Speed of the HOT Lanes and the GPLs................................................79

Safety Implications of the Express Lanes............................................... 80

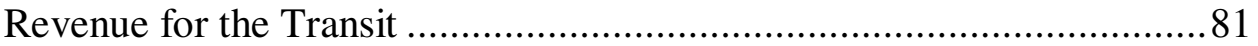

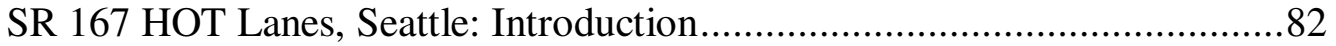

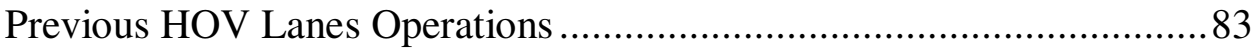

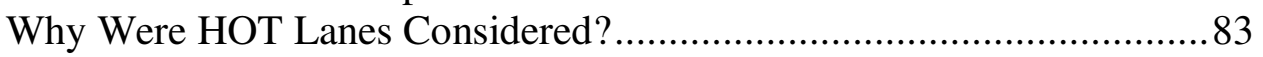

Objectives of the HOT Lanes ..............................................................8

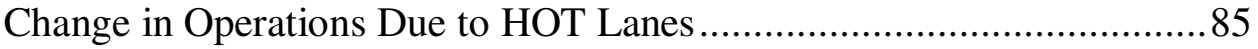

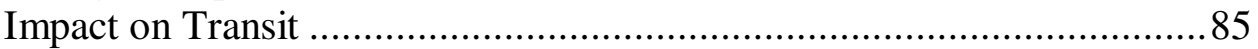

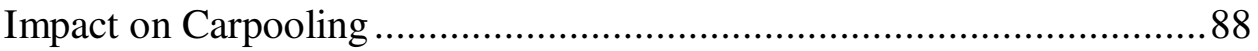

Relationship Between Gas Prices, Tolled Trips and Transit Usage...........90

Usage of the HOT Lanes ......................................................................94

Safety Implications of the HOT Lanes ................................................94

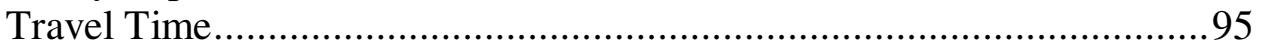

SR 167 HOT Lanes and I-394 Diamond Section.....................................95

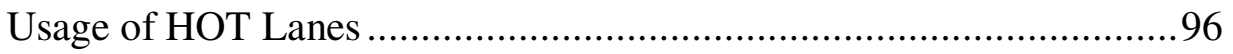

Safety Implications of the HOT Lanes ..............................................97

CHAPTER VI I-10 AND US 290 HOT LANES, HOUSTON ………………....................99

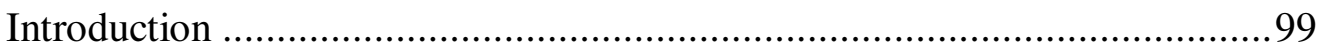

Why Were HOT Lanes Considered?...................................................... 100

Katy Freeway HOT Lane................................................................. 100 
Northwest Freeway HOT Lane .....................................................102

Objectives of the HOT Lanes ...................................................................... 103

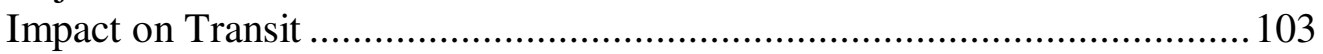

Impact on Carpooling............................................................................. 105

Previous Mode or Mode Used on Non-HOT Lane Trips of Those

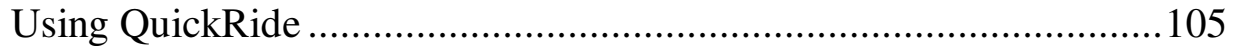

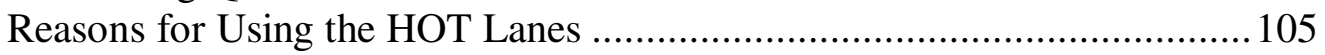

Usage of the HOT Lanes ........................................................................... 105

CHAPTER VII I-15 EXPRESS LANES, SALT LAKE CITY ……....................................108

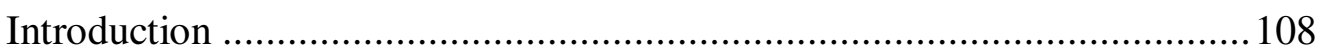

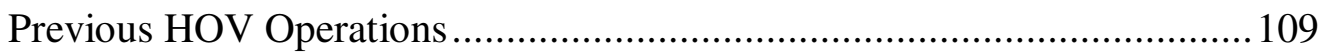

Why Were HOT Lanes Considered?.....................................................110

Objectives of HOT Lanes........................................................................

Changes in the Corridor Due to HOT Lanes .............................................110

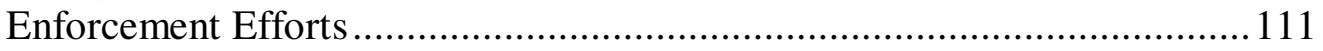

Perceptions of Access Points and Safety of the Express Lanes .....................111

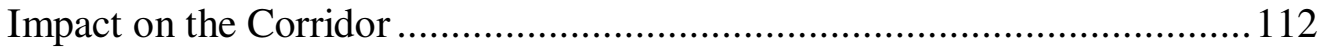

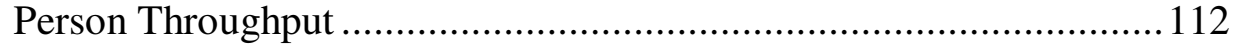

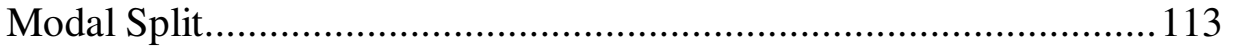

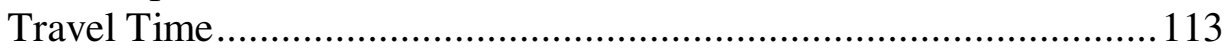

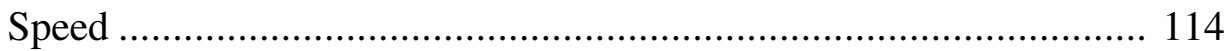

CHAPTER VIII CONCLUSIONS AND RECOMMENDATIONS …….............................115

SR 91 Express Lanes and I-95 Express Lanes............................................115

I-15, San Diego and I-25, Denver Express Lanes........................................117

I-394 HOT Lanes (Diamond Lane Section) and SR 167 HOT Lanes ........... 119

I-10 and US 290 HOT Lanes in Houston .................................................. 120

I-15 Express Lanes in Salt Lake City..................................................... 120

Safety Implications of the HOT Lanes.......................................................121

Impacts of the Different HOT Lanes ......................................................... 130

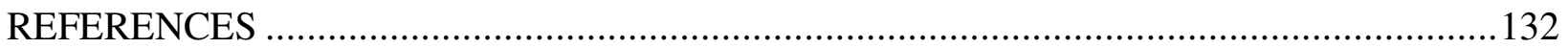

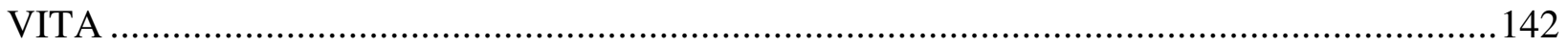




\section{LIST OF FIGURES}

Page

Figure 1 Fully Operational and Planned HOT Lane Projects around the Country....................2

Figure 2 Location of SR 91 Express Lanes ..................................................................... 14

Figure 3 Daily Ridership Trend for Metrolink Rail Line and Service (Sullivan, 1998) ............17

Figure 4 Comparison of Express Lane Traffic Growth in Toll Paying and HOV3+

(Sullivan, 1998)

Figure 5 PM Peak Traffic Growth on SR 91 (All Lanes Eastbound) by Occupancy Group

(Sullivan, 1998)

Figure 6 Location of 95 Express Project on I-95 (Source: 95 Express).....

Figure 7 I-15 Corridor Map Showing Original 8 Mile Section (SR 163 to SR 56/Ted

Williams Parkway) and New 4.5 Mile Section (SR 56/Ted Williams Parkway to

Rancho Bernardo Road)(Source : www.keepsandiegomoving.com ).....................45

Figure 8 I-15 Express Lanes Daily Average Traffic (SANDAG, 1999)..............................52

Figure 9 Map Showing Location of I-25 Express Lanes (RITA website) ............................55

Figure 10 Usage of Express Lanes and Gas Prices (June 2006 Through July 2009) ................61

Figure 11 Unemployment Rate for the Denver Region (source: www. Monster.com) ..............63

Figure 12 I-394 West Section from Wayzata Boulevard to TH 169

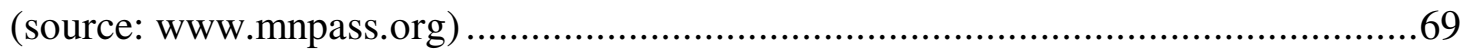

Figure 13 I-394 East Section from General Mills Boulevard (source: www.mnpass.org) ...........70

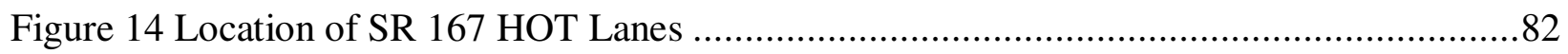

Figure 15 Average Weekday Ridership of the SR 167 Corridor (WSDOT, 2010) ..................87

Figure 16 Weekly US Retail Gasoline Prices (EIA website) .............................................91 
Figure 17 Weekday Transit Ridership (WSDOT. 2010)....................................................92

Figure 18 SR 167 HOT Lanes Average Daily Tolled Trips (WSDOT, 2010)........................92

Figure 19 Usage of HOT lanes by Toll and HOV Users and Gas Prices for May 2008

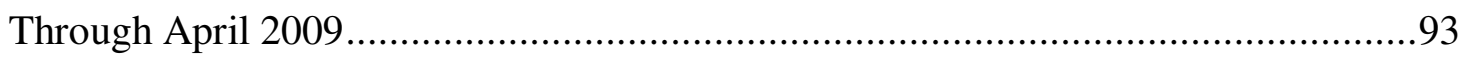

Figure 20 Location of US 290 and I-10 (source: METRO website) .................................. 100

Figure 21 Location of I-15 Utah Express Lanes 38 miles Section from University

Parkway in Orem to 500 North in Salt Lake City (Utah DOT website) 109 


\section{LIST OF TABLES}

Page

Table 1 Speed and Volume of HOV Lanes and GPLs on I-95

(Cambridge Systematics, 2006) .

Table 2 PM Peak Period Travel Speed Comparison- 2008 vs 2009 (Northbound)

(Cain, 2009). .26

Table 3 Average Weekday Boardings- Pre and Post Deployment Comparison

(Northbound and Southbound) (Cain, 2009)

Table 4 Person Throughput by Vehicle Type in Managed Lanes 2008 vs 2009

(Northbound; PM Peak Period- 4 to 6 PM) (Cain, 2009).

Table 5 Mode Used to Access the Express Lanes and Corresponding Prior Modes

(commuters)

Table 6 Mode Used to Access the Express Lanes and Corresponding Prior Modes (non-

commuters)

Table 7 Usual Mode of Express Lane Users and Their Prior Mode. .31

Table 8 Usual Mode of Express Lane Users and Their Mode to Access Express Lanes...........31

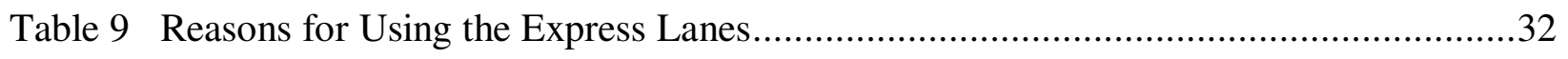

Table 10 Percentage of Respondents by Their Prior Mode in HOV Lanes ................................33

Table 11 Reasons for Not Using the Express Lanes by Previous Carpoolers in HOV Lanes ......33

Table 12 Previous Mode for Converted FasTrak Customers and Other I-15 Users...................50

Table 13 Toll Schedule for I-25 Express Lanes ..............................................................58

Table 14 Average Weekday Ridership for Selected Denver Transit Routes ...........................59 
Table 15 Average Peak Period Transit Ridership for Third Quarter (3Q) on Minneapolis

HOV and HOT Lanes (Mn/DOT, unpublished data). ..................................... 74

Table 16 Comparison of Usage and Revenue Before and After Rate Change..........................77

Table 17 Comparison of Person Throughput and Vehicle Throughput Before and After

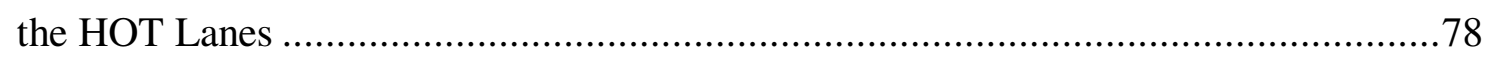

Table 18 Comparison of Pre- and Post- MnPASS Speeds in the MnPASS Lanes

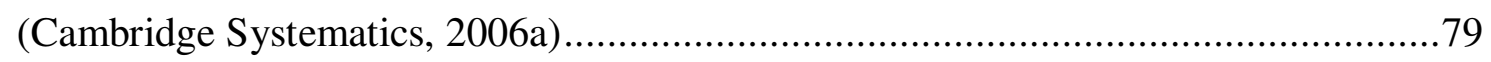

Table 19 Comparison of Pre- and Post- MnPASS Average Speeds on the GPLs

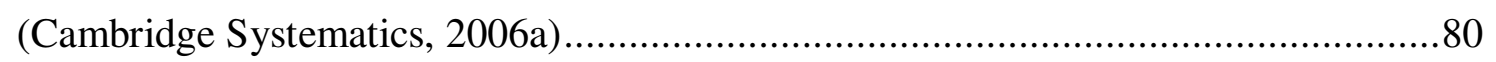

Table 20 Average Weekday Ridership for Express Lanes Bus Routes

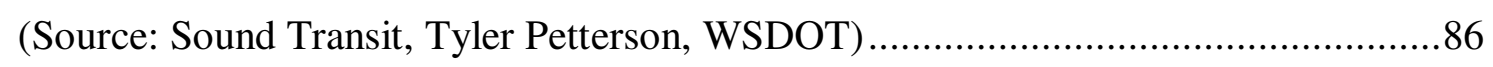

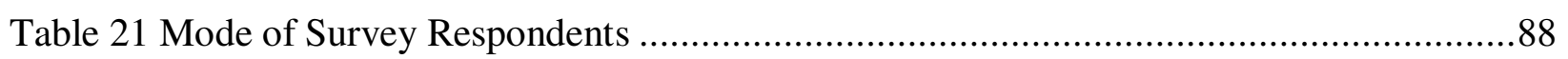

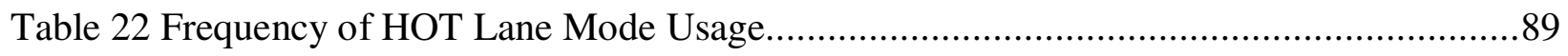

Table 23 Toll, and HOV usage of HOT Lanes and GPL Volume.......................................96

Table 24 Complaints about METRO Service ......................................................... 104

Table 25 Total US 290 HOV/HOT Lane Volume for Morning Peak (6:45-8:00) ...................106

Table 26 Mode Split in the Express Lanes in the AM and PM Peak Hours (March through

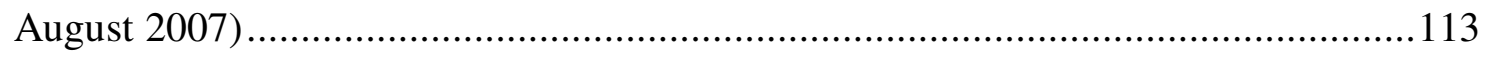

Table 27 Travel Time Summary for May, June, July and August 2007 ..............................113

Table 28 Percent of Congested Travel, Average and Standard Deviation of Speeds ................114

Table 29 Separation Mechanism and Safety Perception of the HOT Lane Users....................122

Table 30 HOT Lane Facilities, Their Objectives, Policy Changes and Impacts .....................125 


\section{CHAPTER I \\ INTRODUCTION}

\section{Background}

High Occupancy Vehicle (HOV) and High Occupancy / Toll (HOT) facilities represent approaches used in metropolitan areas throughout the county to help address traffic congestion, mobility, and air quality concerns. These lanes usually offer travelers reduced travel times that are more reliable, thus encouraging them to carpool, vanpool, or ride the bus. In turn, this can increase the people-moving capacity of the travel corridor. HOT projects provide additional travel options by expanding the user groups allowed on the HOV lane to include solo drivers or lower-occupant vehicles, who can access the lanes by paying a fee. The development and operation of HOV and HOT facilities have evolved over the past 40 years. The opening of the bus-only lane on the Shirley Highway (I-395) in northern Virginia in 1969 and the contraflow bus lane on the approach to New York-New Jersey's Lincoln Tunnel in 1970 represent the first freeway HOV applications in the country (Turnbull, 2002).

Over the past 15 years the country has witnessed a handful of the High-Occupancy Vehicle (HOV) lanes being converted to High/Occupancy toll (HOT) lanes, allowing lower occupancy vehicles (generally, single occupants) to access the carpool lanes by paying a fee while higher occupant vehicles continue to access the lanes for free. In December 1995, the nation's first implementation of High Occupancy/Toll (HOT) lane opened. This project was the 91 Express Lanes, where carpools with three or more passengers could use the lanes for free (excepting the period from 1998 to 2003 when HOV3+ were required to pay half the SOV toll). This project was followed by nine HOT lanes converted from HOV lanes across the United States. These projects included (with year of conversion in parentheses) I-15 Express Lanes in San Diego, California (1996), Katy (1998) and Northwest (1999) Freeways in Houston ,Texas, I-394 in Minneapolis (2005) and I-35W (2009), Minnesota, I-25 in Denver, Colorado (2006), I-15 in Salt Lake City, Utah (2006), and more recently, SR-167 in Seattle, Washington (2008), and I-95 in

This thesis follows the style of Transportation Research Part A. 
Miami, Florida (2008). As of September 2009 there were ten fully operational HOT lanes. Figure 1 shows the current operational and planned HOT lane projects around the country. According to the U.S. Department of Transportation's 1995 Nationwide Personal Transportation Survey, the average occupancy of vehicles in metropolitan areas dropped from 1.17 persons per car in 1970 to 1.09 in 1990. According to the 1995 Nationwide Personal Transportation Survey, only 9 percent of work trips are made in multi-occupant vehicles, compared with 16 percent in the 1980s (Poole and Orski, 2000). Poole and Orski (1999) also noted that:

"With work schedules becoming more flexible, travel patterns more complex, "trip chaining" more prevalent, and homes and jobs dispersing to far-flung suburban locations, fewer commuters are taking advantage of carpool lanes.”

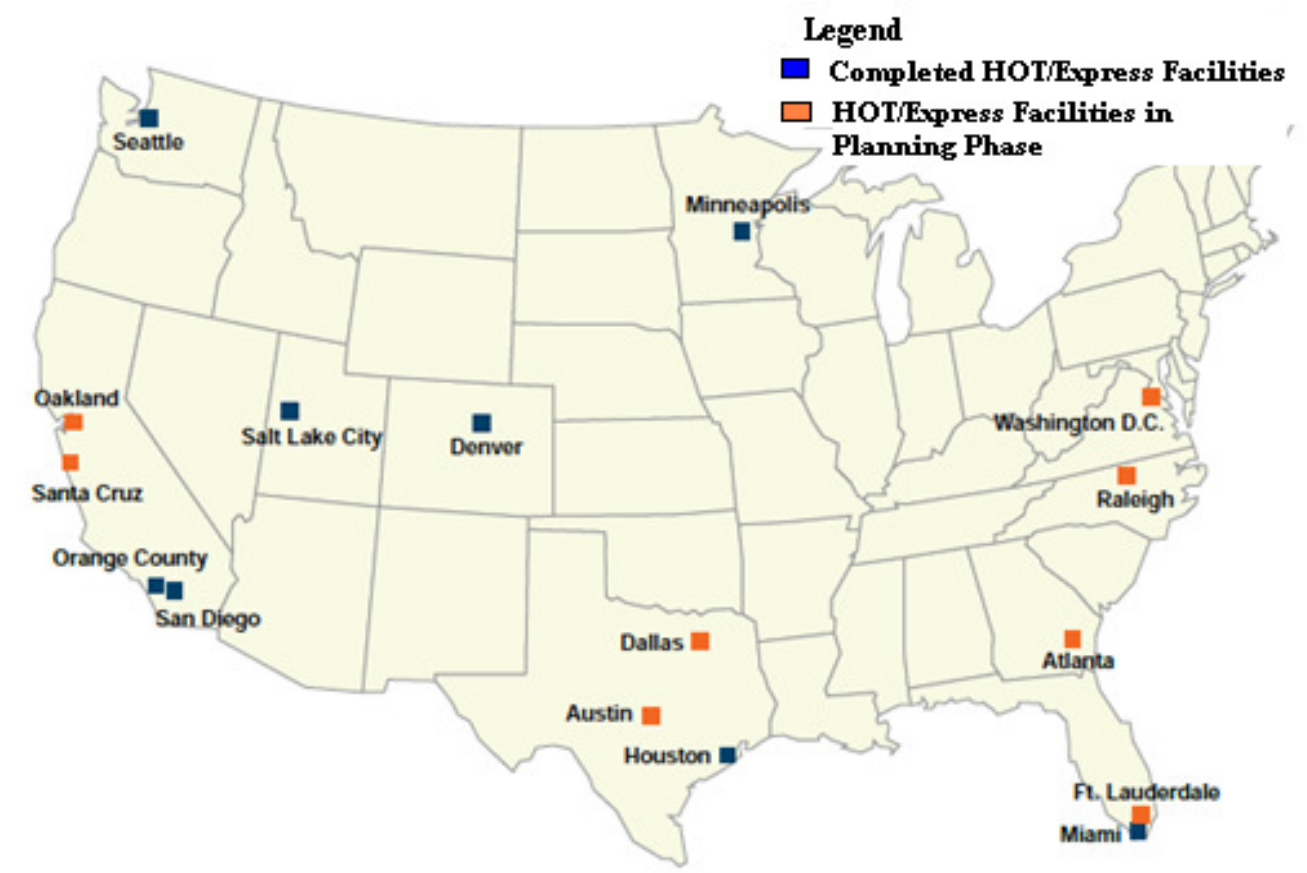

Figure 1 Fully Operational and Planned HOT Lane Projects around the Country

(Stone, 2009)

This decreasing trend of carpooling is reflected in the underutilization of many HOV lanes around the country. In fact, in most of the cases the concerned authorities considered the conversion of HOV lanes to HOT lanes due to excess available capacity of HOV lanes. For instance Tone (2009) mentioned that even by 2025, HOVs on the I-25 HOV lanes in Denver 
would only use half of the available capacity. The carpool lanes on I-15, Utah were underutilized and averaged less than half the capacity (number of cars per hour) they could handle normally (Vladisavljevic et al., 2008). When residents perceived that I-394 HOV lanes were not being efficiently used MnDOT was directed by the Minnesota legislature to consider alternatives including conversion to HOT lanes (Buckeye and Munnich, 2004). The San Diego Association of Governments considered converting I-15 HOV lanes to HOT lanes because of the inefficient utilization of carpool lanes since the lanes opened in 1988 (Poole and Orski, 2000).

The choice between HOV lanes or HOT lanes is generally straightforward - the HOT lanes should provide greater benefits. Generally speaking, the HOT lanes provide the benefits of HOV lanes plus allowing additional vehicles to fill unused HOV lane capacity, all while keeping travel speeds at an acceptable level. When examined closely, there are some issues, positive and negative, involved with HOT lanes which make it difficult to make a blanket statement regarding the use of HOT lanes. The following points summarize the possible benefits of HOT lanes as noted by Poole and Orski (1999):

- HOT lanes provide better utilization of HOV lanes. As noted above, most of the HOT lanes were converted from HOV lanes because of the unused capacity on the HOV lanes.

- HOT lanes provide premium service to those who are in need of a fast, reliable trip and are willing to pay.

- HOT lanes can reduce traffic congestion in the General Purpose Lanes (GPLs) by diverting some single occupant vehicles (SOVs) to HOT lanes thus benefitting both the shifted SOV drivers as well as GPL drivers.

- Revenue from HOT lanes can be utilized to finance public transportation in the corridor.

In addition, because of the revenue generating nature of HOT lanes they can be self sufficient. For instance, a feasibility study comparing the options of adding HOV lanes or HOT lanes to the median of US 101 freeway in Sonoma county, California found that in contrast to a HOV lane, a HOT lane option with a variable pricing can be added with no cost to taxpayers because the revenue generated from HOT lanes is sufficient to cover both the capital and operating costs (Poole and Orski, 2002). Setting the HOT lane toll can serve three purposes: maximizing the efficiency of the HOT lane, maintaining free-flow speeds for HOVs, and generating revenue. To 
meet the first goal, tolls must not be set too high. To meet the second, they must not be set too low. To meet the third, they should be set so as to generate the maximum revenue (Dahlgren, 1999). Therefore, the ultimate decision regarding the toll structure depends on the underlying policies of the decision makers, which might vary because of different weights given to the three factors.

On the other hand, there are some challenges related to HOT lanes. They are as follows:

- HOT lanes are often said to benefit wealthy people since they are the ones who could afford to pay tolls to access HOT lanes and thus giving rise to an equity issue.

- Some of the previous carpoolers/ transit users might shift to paying SOVs in HOT lanes which might result in an overall increase in congestion as well as emissions.

- HOT lanes may have a negative effect on casual carpooling ${ }^{1}$ since some drivers previously picking up the slugs might shift to toll paying SOVs leaving fewer vehicles for casual carpools (Safirova et al. 2003, Burris and Winn 2006).

- As more and more SOVs begin to access HOT lanes it will lengthen the travel time of carpoolers due to increasing congestion and they will see no more incentive in carpooling (Safirova et al. 2003).

Conversion of an HOV lane to a HOT lane generally imposes costs for (Dahlgren 1999):

- design and environmental assessment,

- any additional right-of-way and paving required for the toll collection system,

- installation and operation of the automated toll collection system,

- transponders located in the vehicles,

- any additional enforcement

Among the issues mentioned above, the issue of mode shifts is critical in estimating the benefits from the HOT lanes. With HOT lanes, a traveler generally has five options: carpool in GPLs, carpool in HOT lanes, bus in HOT/GP lanes, SOV in GPLs and SOV in HOT lanes. The

\footnotetext{
${ }^{1}$ Casual Carpooling or "slugging" consists of impromptu carpools formed among strangers to meet the occupancy requirement of HOV/HOT lanes (Burris and Winn, 2006). The passengers picked up by a driver to form a carpool are called slugs.
} 
availability of all these options to the travelers makes the evaluation of a HOT lane project complex. And the different mode shifts among these modes decide the benefits achieved from the HOT lanes. For example, SOVs from the GPLs shifting to HOT lanes would benefit both the shifted SOV traveler and the GPL travelers by reducing congestion on the GPLs. On the other hand, a shift from HOVs or transit to SOVs on HOT lanes would result in the increased congestion and hence the vehicle emissions.

Since the implementation of the first HOT lanes on SR 91 this issue has been extensively studied by many researchers around the country. The research mainly focused on how the conversion of HOV lanes to HOT lanes affects travelers' behavior in the corridors and how demographic and trip characteristics play an important role in the choice of a traveler's mode (Parkany 1999, Sullivan 2000, Ghosh 2000, Burris and Appiah 2004, Chum and Burris 2008). Researchers have used different approaches, such as using theoretical mode choice models based on a survey of the travelers or from the real time traffic counts which include vehicle occupancy information.

\section{Problem Statement}

HOT lanes are gaining interest throughout the country as a strategy for meeting multiple performance objectives in congested urban freeway corridors. HOT lanes can provide benefits in reducing travel time, offering travelers viable options to congestion, improving freeway efficiency, increasing the attractiveness of alternative modes, and raising revenue to offset implementation and operating costs. However, the extent to which these benefits can be attained depends on different policies of the HOT lanes.

With ten fully operational HOT lanes already in place, there is an opportunity to learn how different factors related to HOT lanes as well as mode choice by the travelers could impact the usage of HOT lanes and how different HOT lane policies adopted during the conversion process affect the HOT lane operation which would further guide the future HOT lane projects. Out of ten, this research examines the nine HOT lanes and excludes the most recent I-35W HOT lanes in Minneapolis. 


\section{Research Objectives}

During the planning process of the HOT lanes, the policies of the concerned authorities (e.g. stake holders, transit advocacy groups, DOTs) influence the formation of objectives of the HOT lanes. This research will examine how those policies influenced the formation of objectives and how those objectives further impacted the operation of HOT lanes. For example, to protect the interest of transit users, the I-25 Express Lanes in Denver specified a hierarchy level for the users of HOT lanes with buses as the highest priority followed by vanpools and HOV3+; HOV2+, motorcycles, hybrid vehicles, and toll paying SOVs. The implication is that toll triggers in the Express Lanes are based on maintaining the free flow travel time of transit (8 minutes) and minimum peak hour toll rates are set to be equal to the bus fare. Also, the travel speed of buses is the primary performance measure of the Express Lanes.

The following are the different HOT lane issues and their descriptions which have been investigated in this research. However, depending on the availability of the information for different HOT lanes, one or more of them may not be part of each HOT lane:

- Why were HOT lanes considered?: The need for HOT lanes arises mostly due to inefficient utilization of the HOT lanes and congestion on the adjacent GPLs. Some authorities consider HOT lane conversion of an under-utilized HOV lane in order to test the abilities of the different operational features of HOT lanes- to help alleviate congestion on their respective corridors -and then use it as a tool to alleviate regional congestion.

- Vehicle Occupancy Requirement: Eligibility requirement for free carpool access is the most important element of planning of HOT lanes. It is used as a policy to manage the existing/future demand for the access to HOV lanes. For example, if a corridor has a high HOV2 volume, then a 2+ carpool requirement will possibly overcrowd the HOT lanes and little capacity will be left for additional toll users.

Hybrid vehicles are also considered by some HOT lanes for free access. From a policy perspective, free access to hybrid vehicles would encourage environment friendly modes. From a traffic perspective a large number of hybrid vehicles accessing the HOT lanes will contradict the basic principle of $\mathrm{HOV} / \mathrm{HOT}$ lanes that is providing free flow travel to higher occupancy vehicles and toll paying vehicles. Out of the nine HOT lanes only I-15 
in Salt Lake City, I-15 in San Diego, I-95 in Miami and I-25 in Denver allow Hybrid vehicles to have free access.

- Access Points: Access of HOT lanes is one of the major issues during the conversion process. It directly impacts the convenience (and safety) with which travelers access the HOT lanes, as well as enforcement efforts. With HOV lanes, the enforcement mostly requires ensuring the number of drivers meet carpool eligibility. However, with HOT lanes, the enforcement efforts increase in order to ensure the carpool eligibility as well as LOVs being charged accordingly. As the number of access points increases the need of enforcement at those points also increases.

- Revenue Generation: Revenue generation mainly depends on the pricing mechanism and the usage of HOT lanes by paying users. Depending on the HOT lanes, the revenue generation objective (maximizing or just enough to cover the operational expenses) as well as its usage (transit development, HOT lane development) vary.

- Toll Rates: Toll rates directly affect the out-of-pocket cost of the toll paying LOVs because of which it is used as a tool to control the number of toll paying users and maintain the free flow of travel on the lanes. However, depending on the pricing mechanism (flat per trip fee, fixed variable or dynamic) the effectiveness of controlling access by toll paying LOVs vary. In addition to controlling the number of LOVs it has a direct affect on the revenue generation of HOT lanes.

- Safety: Safety issues with HOT lanes depend mostly on the separation mechanism of HOT lanes, and the number of access points. For example, safety issues are fewer with concrete barrier separated HOT lanes with no intermediate access points and will be greater with double-white line separation and many intermediate access points. Though, safety is not usually mentioned as one of the objectives of HOT lanes however, safety is an important aspect of any highway project thus is considered to be an important performance measure.

\section{Research Methodology}

This research examines the nine different HOT lanes, beginning with their planning stage and ending after their operation starts. Depending on the availability of information for the different HOT lanes, this research examines the corridor conditions (HOV lanes as well as the GPLs) 
which lead to the consideration of HOT lanes in the corridor. The research also examines the objectives of the HOT lanes, the changes made in the HOV lanes or the corridor due to HOT lane operations and finally the different impacts due to the HOT lanes. The impacts have been examined based on both surveys of travelers in the corridor as well as the traffic, transit and ridesharing changes in the corridor after the HOT lane was implemented. An important part of this research is to investigate these different impacts in the context of HOT lanes policies.

\section{Thesis Organization}

This thesis is divided in to eight chapters. Chapter I focuses on the introduction of HOT lane concept; its importance in the existing scenario of high transportation demand and limited infrastructure supply; and the present status of HOT lane projects in the country. It also discusses various benefits, costs and negative impacts of HOT lanes. The chapter also includes the research problem, research objectives and the methodology. Chapter II is the literature review of some of the available research on HOT lanes. Different research, investigating the impacts of HOT lane implementation as well as the different factors impacting the usage of HOT lanes, have been discussed. Following this, the next 3 chapters (III, IV and V) discuss the planning, objectives, operations and impacts of different HOT lanes. Each chapter includes two HOT lanes with similar design and operational features. The chapters also include the comparisons of the different impacts HOT lane implementation had in their respective corridors. Chapter III focuses on the SR 91 Express Lanes in Los Angeles and I-95 Express Lanes in Miami. Chapter IV focuses on the I-15 Express Lanes in San Diego and I-25 Express Lanes in Denver. Chapter V focuses on the I-394 HOT lanes in Minneapolis and SR 167 HOT lanes in Seattle. The next two chapters, VI and VII, individually examine the I-10 and US 290 HOT lanes in Houston and I-15 Express Lanes in Salt Lake City. The two HOT lanes were discussed separately because of difference in their design and operational features from other HOT lanes. Finally, chapter VIII discusses the different findings from the research and their implications in the planning of future HOT lanes. 


\section{CHAPTER II}

\section{LITERATURE REVIEW}

Many researchers have evaluated the impacts of HOT lane implementation in different corridors. Parkany (1999), Mastako et al. (1998), and Sullivan and Harake (1998) investigated the impacts of SR-91 Express Lanes on carpooling rate, changes in ridesharing, and corridor traffic, respectively. Parkany (1998) and Mastako et al. (1998) both concluded that the implementation of HOT lanes did not have any negative effect on carpooling. Later, $\mathrm{Li}$ (2001) also found that pricing incentives of HOT lanes encourages carpooling and does not discourage it. Using the survey data from SR 91 HOT lane travelers found the relation between HOT lane usage and different socioeconomic characteristics of travelers. It was found that household income, vehicle occupancy, trip purpose, and age are important determinants of HOT lane use.

Several researchers have also discussed the policy implications of their research on the decision where and when HOT lanes should be implemented. Li (2001) found the usage of SR 91 Express Lanes dependent upon travelers' characteristics like household income, being on a commuting trip; and also found that the HOT lanes are more likely to succeed in locations where congestion is extreme. Li discussed some policy implications based on those findings including- setting an affordable toll is critical for the success of HOT lanes, policies that provide economic incentives for HOVs should be continued in conjunction with other market-based strategies in order to achieve maximum benefit of transportation investments, and policy makers should pay special attention to the needs of travelers engaging in trips on a daily basis, especially those low-income commuters. Dahlgren (2002) found that a HOT lane is most effective in reducing delay when the initial delay is high and the initial proportion of HOVs is not high; and because of costly construction and operation of HOT lanes, revenue generating potential must also be assessed.

Munnich and Buckeye (2007) discussed feasibility and success of many operational issues related to the implementation of MnPASS in Minnesota. The issues like separation mechanism, safety, use of revenue generated, electronic and dynamic signage, enforcement, effects on carpoolers and transit, and equity concerns were discussed. MnPASS was the first HOT lane project in the country to implement the double striped separation mechanism (SR 91 and Texas 
HOT lanes used buffer and barrier separation respectively). Therefore there was some worry about the effectiveness of this separation mechanism. However, the violation rate has been very low and a bus agency actually reported this separation mechanism as safer than the barrier. A survey of corridor commuters conducted as part of the project evaluation showed that transit users as well as carpoolers support the idea of allowing solo drivers to use the lane for a fee since the HOT lane maintains the LOS as before the conversion. Brownstone et al. (2003) found that the choice of I-15 FasTrak (tolled trip) in San Diego was related to travel time savings and pricing. Golob, 2001 found that the choice of I-15 Express Lanes was related to the level of the perceived safety advantage of I-15 Express Lanes travel.

There is also research available on how HOT lanes impacted public transportation. Turnbull (2008) and Chum and Burris (2008) investigated the impacts of HOT lane on public transportation. Turnbull (2008) underscored the importance of monitoring the effect of HOT lanes on transit operations and ridership. Chum and Burris (2008) found, based on a survey of park-and- ride bus passengers on two Houston freeway corridors, that in a HOT lane scenario very low proportion of transit users would shift to SOVs on HOT lanes and that the average vehicle occupancy is affected more due to SOVs on GPLs shifting to HOT lanes than those shifting from transit. The usage of transit on HOV lanes was examined in the Transit Cooperative Research Program report-12 (TCRP, 2000). This research underscores different characteristics of the HOV facility which could impact the usage of HOV lanes. One of the characteristics was the availability of supporting facilities- park-and-ride lots and parking incentives. The bus ridership on the HOV facility can be enhanced by the availability of parkand-ride lots. The combination of park-and-ride lots and transit facility allows transfer to a highoccupancy mode - rail transit, bus, vanpool, or carpool - where travel densities become higher and more supportive of high-occupancy mode efficiencies (TCRP, 2000). Along with the benefit of accessing the higher occupancy mode, park-and-ride facilities provide incentives of discounted parking cost. For example, the iCommute program in San Diego region provides free parking to carpoolers at different park-and-ride lots along the I-15 Express Lanes (iCommute website). 
Another strategy of parking incentives (in addition to discounted parking at park-and ride lots) is to provide discounted parking for the carpools in the downtown area (TCRP, 2000). Some programs provide discounted parking to carpools in the downtown area. For example ABC Ramps provides parking incentives to the carpoolers traveling eastbound on I-394. For carpoolers the monthly parking pass is $\$ 20$ while it is $\$ 140$ for non carpoolers (ABC ramps website). Seattle Department of Transportation also provides discounted parking to carpools at some designated parking spots in the Seattle downtown area (City of Seattle website). Regional Transit District (RTD) in Denver provides discounted parking to carpoolers as well as transit users whose vehicles are registered within RTD's service boundary (RTD-Denver website).

The TCRP-12 research focused on only the HOV lanes. However, carpools and transit are essential parts of HOT lanes and therefore, the research can be applied to enhance HOT lane strategies as well. Therefore, park-and-ride lots as well as the parking incentives to carpoolers have the potential to increase the usage of HOT lanes by promoting use of carpools and transit. Also, the availability of park-and-ride facilities and their capacity should be examined before expecting any increase in the carpool usage or transit usage on HOT lanes.

An example of an alternative route availability impacting the mode shift in a HOT lane scenario can be seen from the research of Yan et al. (2002). They developed choice models on the basis of data from surveys of SR 91 commuters and found very small shifts to different vehicle occupancies in response to toll changes. They felt this was due to the option of a free parallel road and travelers have a lower need to change their mode occupancy level.

Researchers in the past have examined the relationship between gas prices and the demand of carpooling. Ferguson (1997) examined the decline in gas prices between 1980 and 1990 (45 percent decrease) and the consequent decline in carpooling (35 percent decrease) and mentioned "this naively suggests that the elasticity of demand for carpools with respect to the price of gasoline is a whopping 0.71". Wachs, 2003 in his research report "A dozen reasons for raising gasoline taxes" mentioned "When fuel taxes rise, the price change encourages us to .... Carpool with one another or to switch to public transit to save money". Bomberg and Kockelman, 2007 examined the change in the travel behavior of survey respondents due to a gas price increase in 
2005 in Austin, Texas. In the survey carpooling behavior was observed to increase with increase in gas prices. However, none of the above research was done specifically in the context of $\mathrm{HOV} / \mathrm{HOT}$ lanes but there are indications of carpool increase with an increase in gas prices.

All these research efforts on traveler behavior point toward, in one way or other, the possibility of usage of HOT lanes being dependent on different factors related to HOT lanes (e.g. pricing, safety, travel time savings) and the external factors such as gas prices or the characteristics of travelers in the corresponding corridor. This research investigates how the usage of HOT lanes is dependent on many different factors as well as the policies of the HOT lanes. This will help planners better understand how policies influence the use and ultimately, level of success of HOT lanes. 


\section{CHAPTER III}

\section{SR 91 EXPRESS LANES, LOS ANGELES AND I-95 EXPRESS LANES,}

\section{MIAMI}

This chapter examines two HOT lanes, the SR 91 Express Lanes near Los Angeles and the I-95 Express Lanes near Miami. These were chosen as a pair due to their many similarities.

\section{SR 91 Express Lanes: Introduction}

(Note: This research focuses on the SR91 Express Lanes from December 1995 through January 1998. Till this period HOV3+ could access the lanes for free.)

SR-91 Express Lanes extend 10 miles between the SR 91/SR 55 junction in Anaheim and Orange/Riverside County Line (see Figure 2). The facility has two lanes in each direction, separated from the GPLs by a soft barrier consisting of a painted buffer with plastic pylons. The Express Lanes have no intermediate exits or entrances. Only passenger cars and small trucks are allowed on the Express Lanes. All motor homes and buses, heavy vehicles and vehicles towing trailers are prohibited. Variable tolling is used to manage the Express Lanes to ensure that traffic remains free flowing. SOVs and HOV2s may access the lanes by paying a toll. All users are required to be registered and carry a transponder. Three or more person carpools as well as vanpools could access the Express Lanes for free.

In the first 2 years of operation (1996 and 1997) of the SR-91 Express Lanes, HOV3+ were not required to pay any toll. The initial maximum toll for HOV2s and SOVs of \$2.50 was first increased by $\$ 0.25$ in January 1997 and in September 1997 by additional increment of $\$ 0.20$ and in April 1998 by another $\$ 0.25$ in order to constrain and spread peak patronage. Peak period travel was already approaching the facility's capacity and threatening to undermine California Private Transportation Company (CPTC's) principal product, a congestion - free peak period ride. With increased demand a half price toll was imposed on HOV3+ vehicles for all times of day in January 1998. This continued until 2003 when this facility was purchased by a public agency, the Orange County Transportation Authority (OCTA). OCTA removed the toll for 
HOV3+ vehicles except between 4 PM and 6 PM eastbound when they pay 50 percent of the full toll. The SR-91 express lane toll structure is complex, varying by hour, by day and by travel direction across different toll levels from $\$ 1.30$ to $\$ 9.9$ (effective July 2009) (Sullivan and Harake, 1998).

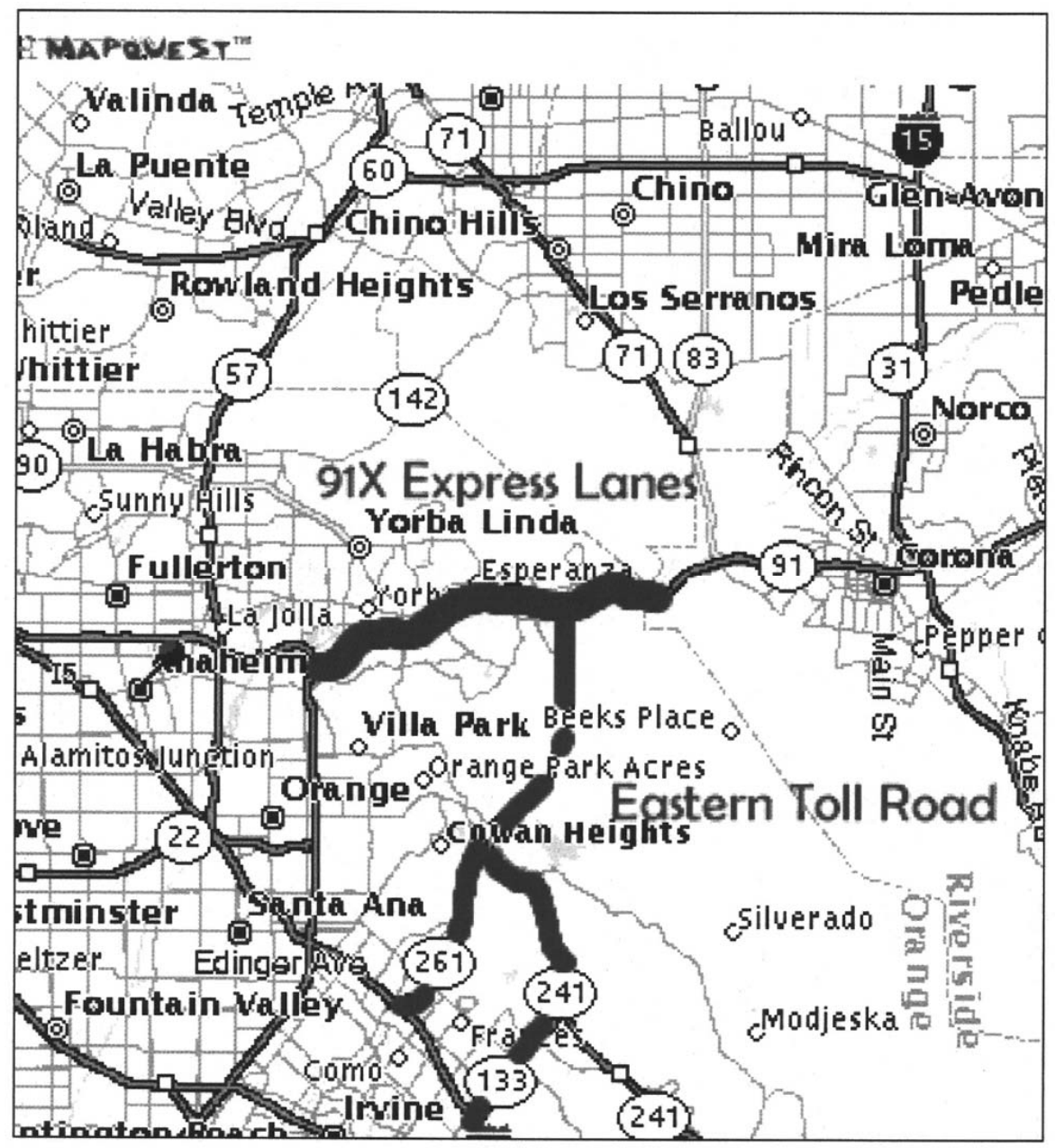

Figure 2 Location of SR 91 Express Lanes

\section{Why Were HOT Lanes Considered?}

SR 91 Express lane facility was built as a private, for-profit, investment as one of four such private-public partnership experiments authorized by the California Legislature under the $\mathrm{AB}$ 680 legislation (Sullivan and Harake, 1998). The primary objective of AB 680 was to test the market feasibility of financing and building new toll roads that could incorporate state-of-the-art automated toll collection technology without the use of public funds (Kim, 2000). 
Explosive subdivision growth in western Riverside County fueled a population boom in the 1980s that caused unexpected increase in peak traffic on SR 91 (Kim, 2000). "The express lanes were built within what had been one of the most heavily congested freeway corridors of California, with typical peak period delays of 30-40 minutes" (Sullivan, 1998).

\section{Policy of 3+ Vehicle Occupancy Requirement}

During the planning phase of the Express lanes, Riverside County Transportation Commission (RCTC), responsible for transportation planning within Riverside County, argued that a toll road would mean double taxation for Riverside county residents. These residents were already paying sales tax for the HOV lanes and would be subjected to a toll to continue west to Orange County on the HOV lanes. RCTC lobbied for HOV2+ toll exemption, but CPTC opposed the HOV2+ toll exemption on the grounds that exemption of HOV2+ would undermine the financial viability of their project. Finally, CPTC and RCTC agreed to a compromise allowing toll exemption of HOV3+. With the agreement, there was also a condition that CPTC could unilaterally charge a half toll to HOV3+ if future growth merited a change in toll pricing (Kim, 2000).

RCTC also opposed the decision of Caltrans and CPTC to have no intermediate access points but agreed with it on the condition that Caltrans and CPTC would provide assurances that ingress/egress points would be built at some future date (Kim, 2000).

\section{Impact on Transit}

Public transit services operating on the SR 91 corridor include the Route 149 express bus service and the Inland Empire - Orange County (IEOC) commuter rail line. The commuter rail line started in October 1996, two months before the Express Lanes started. Unlike all the other HOT lanes, the express bus on SR 91 corridor never uses the Express Lanes. Initially the Express Lanes were examined for use by the Express bus but that was not possible without realigning the bus route to the freeway and dropping the local service. Therefore, the express bus continued to serve the local stops and not use the Express Lanes (Sullivan, 1998). 


\section{Changes in Bus Operation after the Express Lanes Opened}

In July 1995, before the Express Lanes started, there were five round trips per day. This was increased to six round trips per day with the addition of one more round trip during the PM peak (the AM peak schedule was unaffected by the 1996 change) after the Express Lanes opened. In June 1997, bus service frequency was again expanded, to seven round trips per day with the addition of another afternoon run. However, the route length in Orange County was cut back to the Mall of Orange, rather than extending to Disneyland as before (Sullivan, 1998).

\section{Rail and Bus Ridership}

The bus ridership both before and after the Express Lanes opened, did not suggest any relation to the opening of the Express Lanes. There was a stable ridership since the Express Lanes started. In late 1996, bus riders were asked if the toll lanes have changed their travel in any way- only 14 percent answered positively and 60 percent answered negatively (Sullivan, 1998).

In a 1996 survey of highway users no respondents shifted to the highway mode from transit. There were 34 bus survey respondents who had commuted in the corridor the year before the Express Lanes started. Over a third of them reported that, at that time, they used the highway mode, split about equally between drive alone and ridesharing (Sullivan, 1998).

However, in case of commuter rail, there were some indications of the Express Lanes operations impacting rail ridership. The trend of the commuter rail line ridership for the two years after its inception (in October 1995) is shown in Figure 3. Two months (October and November - 1995) are before the Express Lanes started. Researchers (Sullivan, 1998) indicated the initial ten months of operation as a flat patronage until it started increasing in September 1996. This increase was attributed to the schedule adjustments.

However, from the survey of SR 91 commuters, there were indications that the rail line commuters used the Express Lanes for some of their trips. In a Fall 1996 survey of rail riders (Sullivan, 1998), out of the 106 respondents who stated they had noticed change in the travel conditions of the corridor after the Express Lanes, 42 percent mentioned there was less 
congestion, 17 percent mentioned congestion shifted elsewhere, 16 percent had a faster/easier commute and 10 percent mentioned Express Lanes are fast. A survey of 350 commuters also revealed that 76 percent of rail riders make use of the Express Lanes for some trips. 73 percent drive alone when they do not use Metrolink and 14 percent carpool (Sullivan, 1998).

Reexamining the trend of rail ridership in Figure 3, in November 1995, the ridership was around 1100 per day. This drops to a little less than 1000 in December 1995 (when HOT lanes started) and keeps decreasing until it reaches a steady ridership of a little more than 650 in May 1996. Therefore, from this decreasing trend of rail ridership after the Express Lanes started as well as survey finding that more than 75 percent of rail riders use the Express Lanes for some of their trips indicate that Express Lanes might have had some negative impacts on rail ridership.

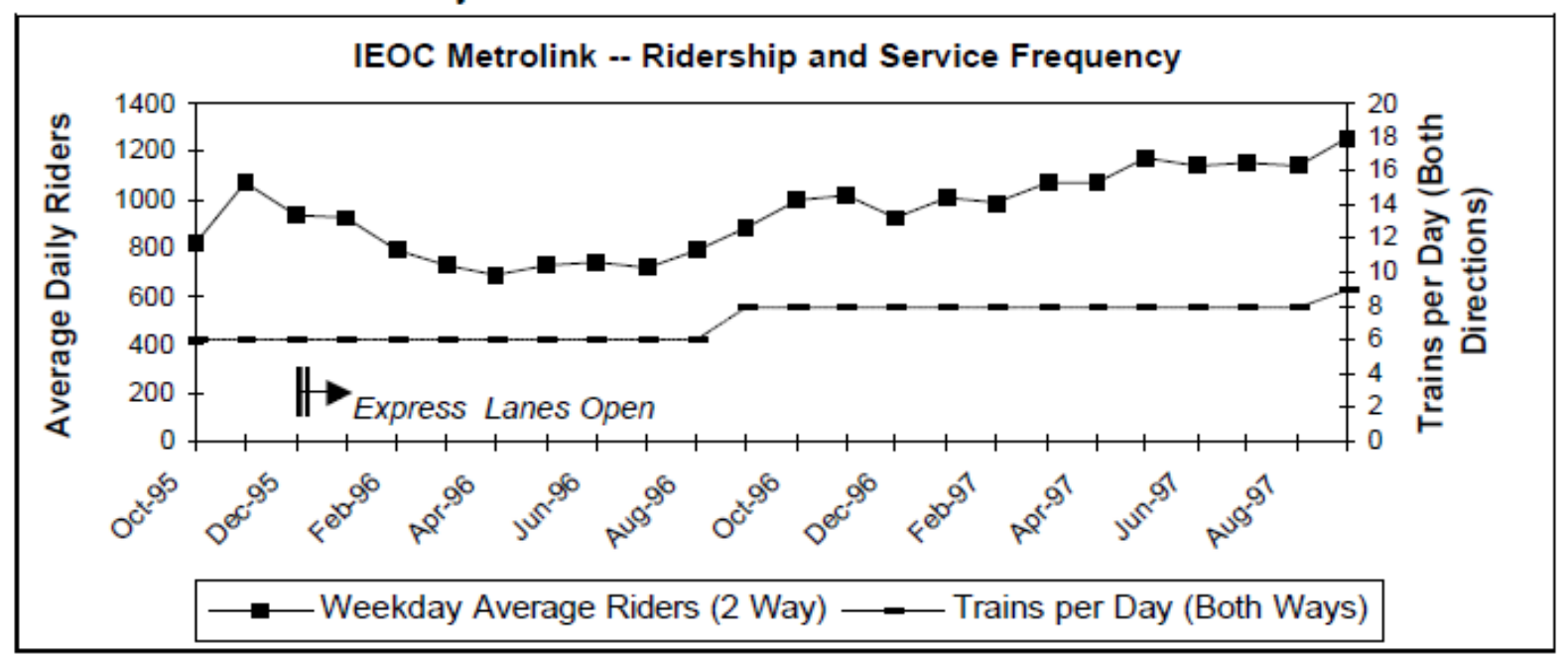

Figure 3 Daily Ridership Trend for Metrolink Rail line and Service (Sullivan, 1998)

\section{Impact on Carpooling}

3-person Carpools

Within three months after the SR 91 Express Lanes opened, peak period HOV3+ travelers increased in the corridor by greater than 40 percent (496 to 725). Note that this increase in HOV3+ traffic is measured relative to a small initial base (see Figure 4). Before the Express 
Lanes opened, HOV-3+ vehicles averaged 3.7 percent of the total SR 91 PM peak traffic in 1995 (Sullivan, 1998).

A year after the Express Lanes opened, there was an increase of 28,000 vehicles per day in the SR 91 corridor. Researchers distributed this increase in traffic among three different groups- (i) travelers who had previously been diverting to parallel arterials and returned to SR 91 because of substantial improvement in travel conditions, (ii) long-term growth trend which existed in the corridor before the Express Lanes, and (iii) induced traffic due to improved travel condition. It was estimated that the induced traffic contributed 60 percent of the ADT increase. It was also estimated that during the PM peak, induced traffic consisted of 12.7 percent of the HOV3+ vehicles. This led to net increases in $\mathrm{HOV}-3+$ percentage compared to pre-opening conditions (Sullivan, 1998). Therefore, in case of SR 91 Express Lanes, increase in HOV3+ traffic can be attributed mainly to the induced traffic.

In contradiction to the increasing number of $\mathrm{HOV} 3+$ in the corridor, there were indications of commuters shifting from higher occupancy to lower occupancy modes. Among the commuters who had been traveling SR 91 before and after the Express Lanes started, the number of commuters who switched from HOVs to SOVs exceeded the number who switched from SOVs to HOVs. This led to a decrease in HOVs of 7 percent (Sullivan, 1998).

\section{2-person Carpools}

Through the eighteen month observation period before the HOT lanes opened, the peak period counts of HOV2 vehicles remained essentially unchanged (see Figure 5). Following the opening of the Express Lanes the trend in HOV-2 traffic showed a comparatively small increase. Researchers examined the usage of conventional HOV lanes by the HOV2s during the 2 hour peak period in the study area. It was found that, during the 2 hour peak period, the usage of SR 91 Express Lanes by the HOV2s was about half (30 percent of the total corridor HOV2s) of that in conventional HOV lanes (60 percent). These observations suggested that opening of the SR 91 Express Lanes did relatively little to encourage or discourage HOV-2 ridesharing (Sullivan, 1998). 


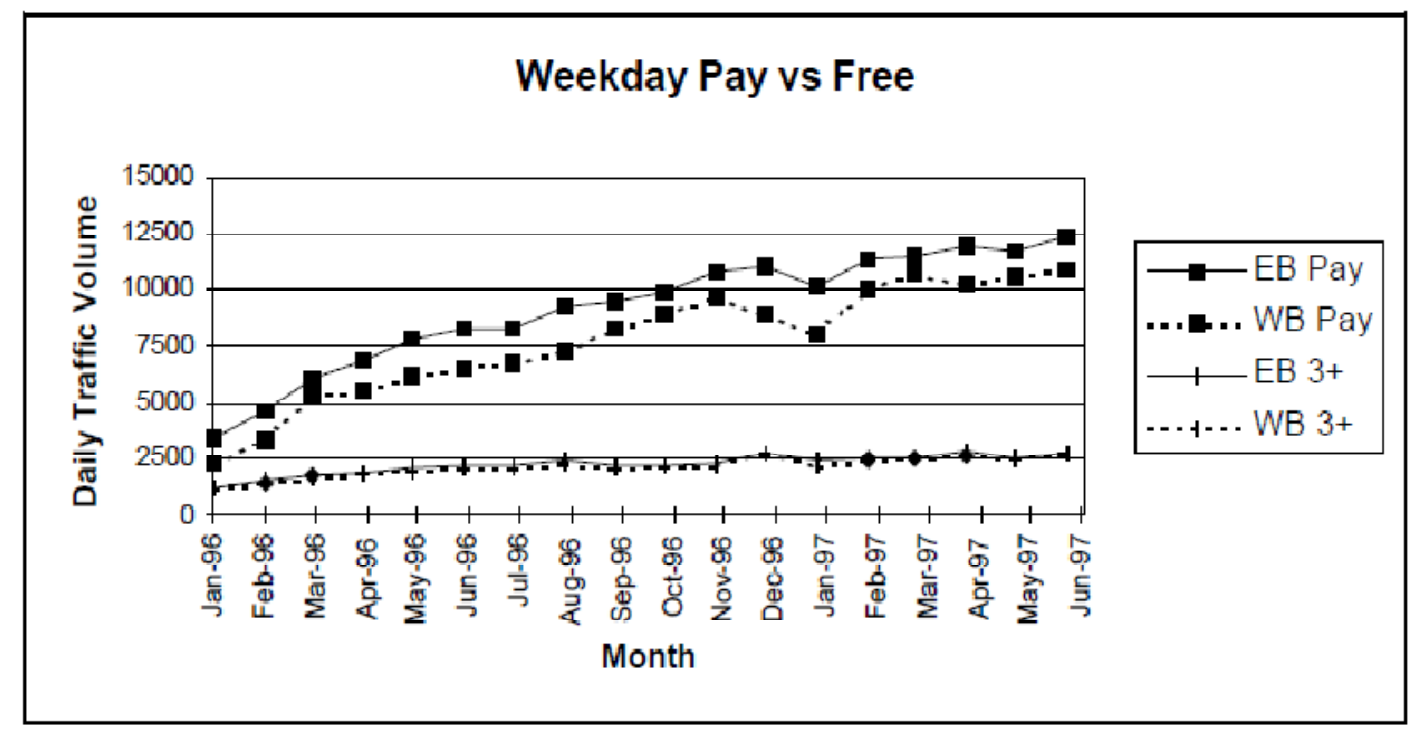

Figure 4 Comparison of Express Lane Traffic Growth in Toll Paying and HOV3+ (Sullivan, 1998)

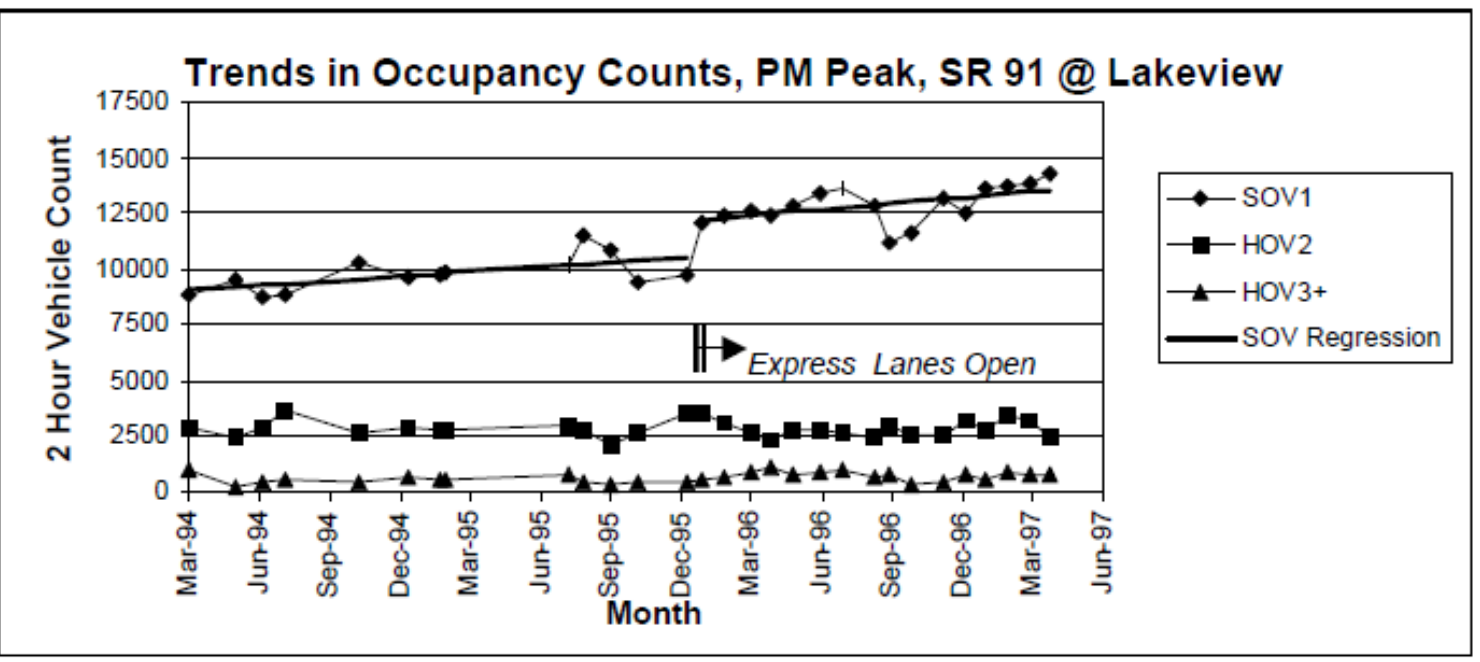

Figure 5 PM Peak Traffic Growth on SR 91 (All Lanes Eastbound) by Occupancy Group (Sullivan, 1998)

\section{Safety Implications of the Express Lanes}

An interesting phenomenon was observed in a fall 1996 survey (Sullivan, 1998) which reflected the perceived level of increased safety with the Express Lanes. Some toll lane users chose to use 
the toll lanes under traffic conditions where their expected value of time savings was clearly less than the toll paid. When respondents were asked different reasons for using the Express Lanes other than the travel time savings, 40 percent of them mentioned driving comfort and the perception of greater safety in the lanes. Some off-peak toll lane use is also probably due to the availability of company-provided transponders.

The accident rate for the section of SR 91 containing the Express Lanes decreased significantly after the Express Lanes opened. This most likely reflected the reduced peak period congestion (Sullivan, 1998).

\section{Travel Time Savings}

In the six months after opening of the Express Lanes, the typical PM peak trip delay on the freeway fell from 30 to 40 minutes to less than 10 minutes per trip. A year later, in June 1997, the PM peak trip delay increased by about 5 minutes to the 12-13 minute range, reflecting both time shifts in travel demand and the effect of the underlying long-term traffic growth trend. A small travel time improvement of about 6 minutes per trip was observed on one of the parallel arterials (Sullivan, 1998).

A strong correlation was found between Express Lane patronage and travel time savings. In spring 1997, the percentage of SR 91 travelers who used the express lanes ranged from about 7 percent in the mid-day off-peak, when time savings were minimal, to a high of 35 percent during the peak hour when delay to freeway users was an estimated 12-13 minutes (Sullivan, 1998).

\section{Induced Traffic}

The total ADT on SR 91 increased by 14 percent one year after the capacity increase resulting from opening the Express Lanes. This increase of about 28,000 vehicles per day (vpd) is approximately equal to the amount that average weekday Express lane traffic grew during the same time period. According to an estimate, more than 50 percent of that increase in ADT was due to induced traffic which shifted to SR 91 because of improved condition and 20 percent due to traffic returning from parallel city streets who previously used the $\mathrm{R} 91$ corridor. These findings also explain the degrading travel time saving values over the first year (Sullivan, 1998). 
Both the city streets and induced traffic consisted primarily (over 80 percent) of SOVs. Not surprisingly, the increase in toll paying SOVs in the Express Lanes greatly exceeded the increase in HOV3+ vehicles. Consequently, the average vehicle occupancy (AVO) decreased for both the $\mathrm{AM}$ and PM peak periods through the first eighteen months of Express lane operation (Sullivan, 1998). Therefore, SR 91 provides an interesting case study in which induced traffic plays an important role in deciding the benefits achieved by the HOT lanes.

Unlike all other HOT lanes in the country, Express Lanes in SR 91 were constructed in the corridor with no existing HOV lanes. Therefore, a 50 percent capacity increase (2 lanes in each direction to an existing 4 GPLs in each direction), lead to improved traffic conditions and subsequent induced traffic, much of it is SOVs- but some HOV3+.

\section{I-95 Express Lanes, Miami: Introduction}

The 95 Express Lanes are located on I-95 near Miami. Phase 1A of this project began in December of 2008. The Express Lanes extend from SR-112 to the Golden Glades Interchange (GGI) area just north of NW 151st Street (see Figure 6). With Phase 1A, the 95 Express converted the single northbound HOV lane into two Express Lanes while maintaining the same number of GPLs. SOVs and HOV2s can access the lanes by paying a toll. Tolls vary with the level of congestion with an objective of maintaining the Express Lanes speed at a minimum of $45 \mathrm{mph}$. Registered vanpools, registered 3+ carpools, registered hybrid vehicles and motorcycles can use the express lanes without paying a toll. Buses of several types can also use the Express Lanes toll-free — Miami-Dade County and Broward County express and regular transit, public school buses and over-the-road coaches. Trucks of three or more axles are not allowed to use the Express Lanes.

Phase 1B opened in January 2010 and runs southbound on I-95 from the GGI area to I-395 (see Figure 6). Phase 1B will also extend the northbound Express Lanes further to the south from SR 112 to I-395 by spring 2010. Phase 2 will create HOT lanes in both directions on I-95 between the GGI area in Miami-Dade County and I-595 in Broward County (FDOT, 2009). For the purpose of this research only Phase 1A and its impacts are discussed. 


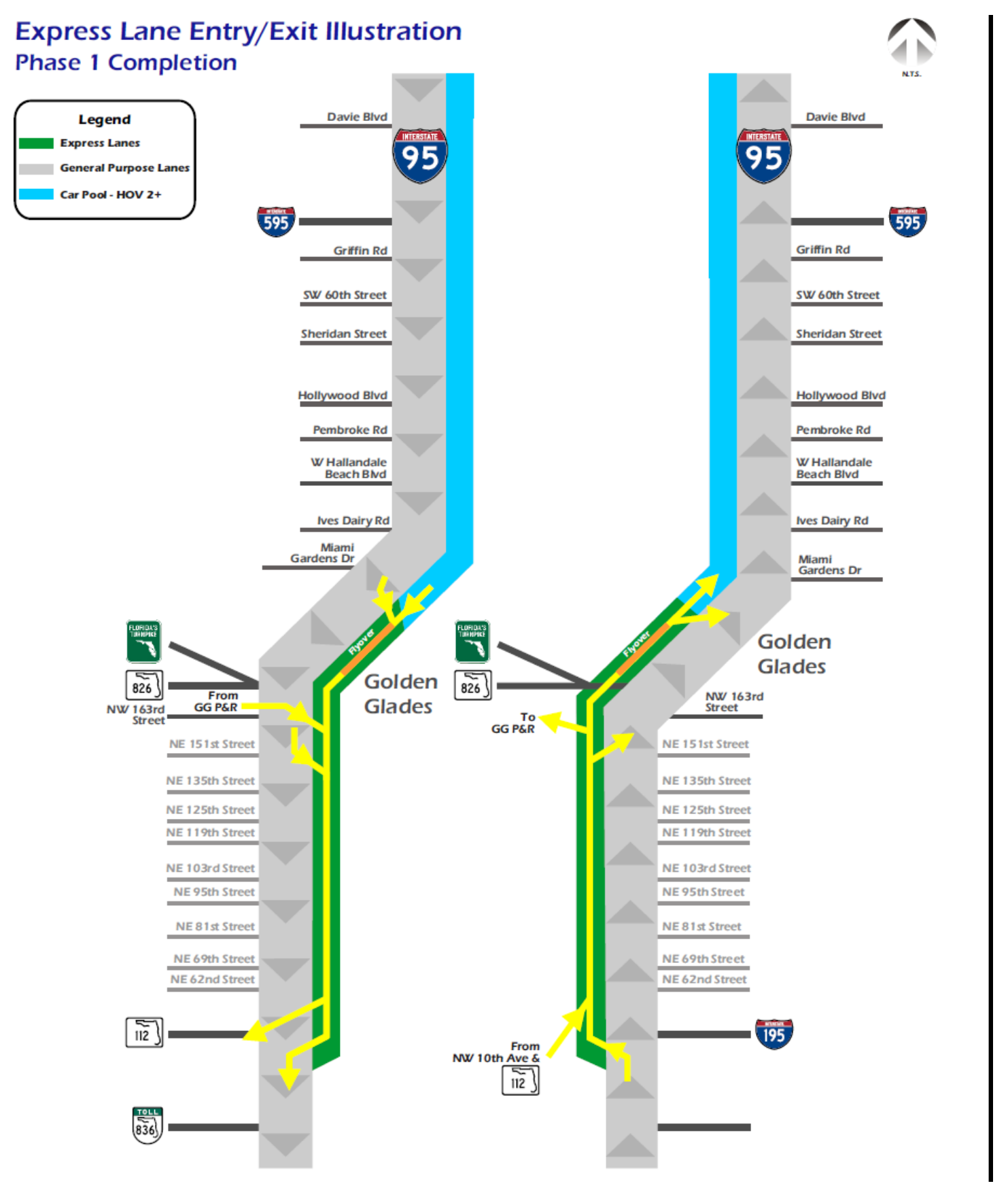

Figure 6 Location of 95 Express Project on I-95 (Source: 95 Express) 


\section{Previous HOV Lane Operations}

Prior to Express Lanes, the corridor's typical cross section provided four GPLs and one HOV lane in each direction. The HOV lanes were separated from the GPLs using double white stripes and HOV lanes could be accessed at any point. The HOV lanes were restricted to HOV2+ and were enforced from 7:00 AM to 9:00 AM and 4:00 PM to 6:00 PM. Express bus service operated along a portion of the corridor providing connections from a commuter rail station/park-n-ride lot to downtown Miami. According to data in 2006, 18 percent of all person trips within this portion of the HOV lanes were associated with the existing bus service. (KimleyHorn, 2008)

South of GGI, the highway carried over $290,000 \mathrm{vpd}$, with traffic volumes expected to exceed 360,000 vpd by year 2030. The volumes and speed for the peak direction of the GPLs and the HOV lanes in 2006, before the implementation of Express Lanes, is shown in Table 1. The HOV lanes performed at a LOS of E or F in Miami-Dade County in the AM peak-period southbound direction and in the PM peak-period northbound direction. (Cambridge Systematics, 2006)

Table 1 Speed and Volume of HOV Lanes and GPLs on I-95 (Cambridge Systematics, 2006)

\begin{tabular}{|l|c|c|c|c|c|c|}
\hline \multirow{2}{*}{ Section } & \multicolumn{2}{|c|}{ Southbound AM Peak } & \multicolumn{3}{c|}{ Northbound PM Peak } \\
\cline { 2 - 7 } & $\begin{array}{c}\text { HOV } \\
\text { Volume } \\
\text { (pc/hr/ln) }\end{array}$ & $\begin{array}{c}\text { HOV } \\
\text { Speed } \\
\text { (mph) }\end{array}$ & $\begin{array}{c}\text { GPLs } \\
\text { Speed } \\
\text { (mph) }\end{array}$ & $\begin{array}{c}\text { HOV } \\
\text { Volume } \\
\text { (pc/hr/ln) }\end{array}$ & $\begin{array}{c}\text { HOV } \\
\text { Speed } \\
\text { (mph) }\end{array}$ & $\begin{array}{c}\text { GPLs } \\
\text { Speed } \\
\text { (mph) }\end{array}$ \\
\hline GGI - 125th Street & 1265 & 31.1 & 13.6 & 1481 & 34.5 & 30.2 \\
\hline 125th street - SR 112 (I-195) & 2058 & 20.6 & 20.6 & 1417 & 23.6 & 14.5 \\
\hline
\end{tabular}

\section{Why Were Express Lanes Considered?}

As described in the section above, the GPLs as well as HOV lanes on I-95 were not able to provide reliable travel. 95 Express Lanes project was designed to reduce congestion and make travel along this portion of I-95 a better experience for drivers, residents, and transit users alike. Ultimately, "it will create more travel options and encourage the use of ridesharing and transit alternates. The first of its kind in the state, this managed lanes project is part of an overall long- 
term strategy of initiatives designed to help improve the safety, throughput and reliability of mobility along the roadways within southeast Florida” (Kimley- Horn, 2008).

The conversion of the I-95 HOV lanes to Express Lanes focuses on the throughput enhancement of the whole I-95 corridor and not only the HOV lanes. Also, it is designed to encourage the use of ridesharing and transit. The preference given to $3+$ carpools probably stems from the objective of encouraging ridesharing.

\section{Changes in the Corridor Due to the Express Lanes}

The project creates an additional travel lane in each direction by narrowing the inside median width and reducing some travel lanes from 12 feet to 11 feet. Additionally, the existing HOV lane buffer was reduced to one foot of separation between GPLs and Express Lanes. The resulting cross section allows for two managed lanes, a one foot buffer (and no median shoulder), and four GPLs in both the northbound and southbound direction. Flexible delineators have been installed within the one foot buffer (FDOT, 2009).

Compared to the previous HOV lanes which could be accessed from anywhere, the Express Lanes have no intermediate access and can be accessed only at the two ends (FDOT, 2009). The project also enhances and expands Bus Rapid Transit service on I-95 from I-395 in downtown Miami to Broward Boulevard in Fort Lauderdale (FDOT, 2009).

The HOT lane operation also includes increasing the carpool eligibility to HOV3+ from HOV2+ in the previous HOV lanes. Also, carpools and vanpools have to be registered to be able to use the HOT lanes for free. The eligibility criteria for a registered 3+ carpool include:

- Participants must live within a 3- mile radius of each other

- Participants must work within one mile radius of each other

- Participants must have start/end work time within 30 minutes of each other (95 Express website) 


\section{Impact on Transit}

The National Bus Rapid Transit Institute (NBRTI) evaluated the transit elements of the 95 Express project after the implementation of Phase 1A of the Express Lanes (Cain, 2009). The data collection was done from January to March 2008 (baseline period) and January to March 2009 (post-deployment period). This allowed the same three months of the year to be evaluated pre-and post deployment, removing the potential for any bias due to seasonal factors. Pre and post deployment on-board surveys were conducted to assess the impact of HOT lanes on transit user perceptions. These surveys were conducted in May 2008 (baseline) and May 2009 (postdeployment).

Despite these efforts, there were some exogenous factors which could impact the travel patterns on the I-95 corridor and hence transit ridership. The factors were (Cain, 2009):

1. Change in the service: There was a reduction in some service from the 95 Express bus in the non-peak direction in June 2008. Some reverse commute direction trips with very low patronage were converted to deadhead trips to save revenue miles.

2. Increase in the bus fare (October 2008): The regular cash fare was increased by 50 cents from $\$ 1.85$ to $\$ 2.35$, representing a 27 percent increase. The cost of the monthly Metropass increased from $\$ 75$ to $\$ 100$, representing a 33.3 percent increase. The discounted pass available through Miami area employers increased from $\$ 65$ to $\$ 85$, representing a 30.8 percent increase.

3. Gas Price Fluctuation: The baseline data collection period occurred when gas prices were around $\$ 3$ per gallon while the 2009 data collection period occurred when gas prices were only $\$ 2$ per gallon. The 2008 on-board survey was conducted during the period of unusually high gas prices when gas prices were over $\$ 4.00$ per gallon. Prices then began to drop in the fall of that year and by the time that Phase 1A opened, prices were under $\$ 2.00$ per gallon. By the time the 2009 survey was conducted in May, gas prices had risen to just over $\$ 2.50$ per gallon.

\section{Travel Time Savings}

The travel speed and travel times in HOV lanes and GPLs before and after the Express Lanes opened are shown in Table 2. 
Table 2 PM Peak Period Travel Speed Comparison- 2008 vs 2009 (Northbound) (Cain, 2009)

\begin{tabular}{|c|c|c|c|c|}
\hline & \multicolumn{2}{|c|}{ Travel Speed (mph) } & \multicolumn{2}{c|}{ Travel Time (min: sec) } \\
\hline & HOV/ HOT & GPL & HOV/ HOT & GPL \\
\hline $\mathbf{2 0 0 8}$ & 18.1 & 18.8 & $25: 02$ & $24: 06$ \\
\hline $\mathbf{2 0 0 9}$ & 56.8 & 39.7 & $7: 59$ & $11: 25$ \\
\hline Change & 38.7 & 20.9 & $-17: 03$ & $-12: 41$ \\
\hline $\begin{array}{c}\text { \% } \\
\text { Change }\end{array}$ & $213 \%$ & $111 \%$ & $-68 \%$ & $-53 \%$ \\
\hline
\end{tabular}

The travel time of vehicles in the HOV lanes decreased from 25 minutes to 8 minutes after the Express Lanes. Since express bus use the Express Lanes, the bus travel time also decreased by 17 minutes.

\section{Ridership}

Miami Dade Transit (MDT) operates express bus service on I-95 Express Lanes known as 95 Express. This express service connects several locations in northern Miami-Dade County with various locations in downtown Miami. There was an increase of 30 percent in the ridership of the express bus service comparing ridership data from January -March 2009 to that of JanuaryMarch 2008 (see Table 3). However, at the corridor level, bus ridership actually dropped by 4.6 percent. This is likely due to small system-wide reductions in service quantity and significant fare increases, coupled with exogenous factors like lower gas prices as described previously as well as economic recession. In addition to those, the 95 Express accounts for less than one fifth of total corridor ridership (the two other routes- 77 and 277 run parallel to I-95 on $7^{\text {th }}$ Avenue). Thus the ridership increase on the express bus was not reflected at the corridor level. The higher income profile of express bus users is one reason why the fare increase has not impacted 95 Express ridership as dramatically as it has impacted the MDT system as a whole. The express bus riders sample has 7 percent of respondents with annual household income less than $\$ 20,000$ while 71 percent of MDT's system wide ridership had annual household incomes under $\$ 20,000$. (Cain, 2009) 
Table 3 Average Weekday Boardings- Pre and Post Deployment Comparison (Northbound and Southbound) (Cain, 2009)

\begin{tabular}{|l|c|c|c|}
\hline & Jan 08- Mar 08 & Jan 09- Mar 09 & $\begin{array}{c}\text { \% } \\
\text { Change }\end{array}$ \\
\hline 95 Express & 1,813 & 2,353 & $29.80 \%$ \\
\hline Route 277 & 1,363 & 1,262 & $-7.40 \%$ \\
\hline Route 77 & 10,917 & 9,824 & $-10.00 \%$ \\
\hline Tri Rail UPA Corridor & 2,673 & 2,686 & $0.50 \%$ \\
\hline Total UPA Corridor with Tri-Rail & 16,766 & 16,126 & $-3.80 \%$ \\
\hline $\begin{array}{l}\text { Total UPA Corridor without Tri- } \\
\text { Rail }\end{array}$ & 14,093 & 13,439 & $-4.60 \%$ \\
\hline
\end{tabular}

\section{Effect of Gas Prices}

When transit users were asked their reason for using the express bus, 28.4 percent respondents mentioned saving money as one of their reasons in May 2008 while in May 2009 only 13.4 percent mentioned this reason. This difference was attributed to the reduction in gas prices in 2009 and increased MDT bus fare after May 2008. In May 2008, weekly gas prices exceeded \$4 per gallon while prices were only $\$ 2.50$ per gallon in May 2009 (EIA website). The two factors (reduced gas prices and the increased bus fare) reduced the fiscal advantage of using transit because of which the money saving factor of transit became less important.

Looking at all other reasons, it was concluded that after the Express Lanes- 'travel time', 'traffic avoidance' and 'convenience compared to private vehicle' became the most important reasons for using the express bus as compared to 2008 (pre- Express), when saving money was the most important aspect (Cain, 2009) which indicates the improvement in travel conditions on the Express Lanes.

\section{Mode Shift due to Transit}

95 Express bus riders were asked how long they have been traveling by bus and what was their previous mode of travel before using the bus service. 92 percent of respondents (307 out of 334) mentioned they have been traveling the 95 Express bus before the Express Lanes started. Only, 8 percent respondents (27 out of 334) began using the bus after the Express Lanes opened. Among them, 50 percent (13 out of 27) had their previous mode as drive alone and none of them carpooled previously. Therefore, 95 Express bus ridership consisted primarily of those who have 
been using the service prior to Express Lanes implementation and the small mode shift from highway to transit was mostly from SOVs. Note that the number of respondents is too small to make any conclusions. (Cain, 2009)

Respondents were also asked whether or not the opening of the Express Lanes had influenced their decision to ride the 95 Express bus service. 16.4 percent of those respondents (52 out of 315) who have been riding the Express bus before the implementation of Express Lanes stated that their decision to ride the Express Lanes was influenced by the Express lane project. This could mean that these riders are either riding the 95 Express bus more frequently, or have decided to continue using the service while otherwise they would have shifted to other modes. Only 9 users indicated that they started using the bus after the Express Lanes started, with four of these users indicating that the opening of the Express Lanes influenced their decision to ride the 95 Express bus (Cain, 2009)

In May 2009, bus riders were asked their perception of different elements of transit as compared to pre-Express lane implementation. The majority of the respondents mentioned service reliability (55 percent) and travel time ( 75 percent) are better after the Express Lanes opened (Cain, 2009).

The above findings indicate that the improvement in the traffic conditions on the Express Lanes (travel time saving of 17 minutes as compared to pre- Express Lanes) overshadowed the reduced fiscal benefit (due to reduced gas prices and increased bus fare) of using transit. Additionally, the increased ridership on the express bus can be attributed mostly to Express lane implementation.

Finally, it should be noted that most express bus users are commuters on daily round trips, and as such still have to endure high levels of traffic congestion in the southbound direction. Thus, the competitiveness of the express bus service as a round trip commute mode versus the private auto cannot be fully realized until the southbound direction is similarly improved under Phase $1 \mathrm{~B}$ of the project (Cain, 2009). 


\section{Impact on Carpooling}

There was a 4.6 percent increase in the person throughput of the whole corridor (see Table 4). This corresponds well with historical trend from 2006 compared to 2008 (Cain, 2009). This indicates that the 256 percent increase of SOVs in the HOV lanes is mostly due to the mode shift from within the corridor and not due to the overall increase in travelers. The overall decrease in the number of HOV2 person volume shows that these carpools either shifted to SOV mode (an overall 33 percent increase in SOVs) or they shifted to higher occupancy (overall 9.6 percent increase in HOV3). The decrease in HOV2 person volume in managed lanes could be because of the toll imposed on them for Express lane use, and the access points reduced to just either end of the facility. However, the decrease in access points would also affect the HOV3 vehicle volumes in the Express Lanes and in place of tolls they have strict guidelines for carpool registration. This mode shift will be examined in the following sections.

Table 4 Person Throughput by Vehicle Type in Managed Lanes 2008 vs 2009 (Northbound; PM Peak Period- 4 to 6 PM) (Cain, 2009)

\begin{tabular}{|l|c|c|c|c|c|c|}
\hline \multirow{2}{*}{ Vehicle Type } & \multicolumn{3}{|c|}{ Managed Lanes } & \multicolumn{3}{c|}{ Facility (GPLs + Express) } \\
\cline { 2 - 7 } & \multicolumn{3}{|c|}{$\begin{array}{c}\text { Total Person Volume per Peak } \\
\text { Period }\end{array}$} & \multicolumn{3}{c|}{$\begin{array}{c}\text { Total Person Volume per Peak } \\
\text { Period }\end{array}$} \\
\cline { 2 - 7 } & $\mathbf{2 0 0 8}$ & $\mathbf{2 0 0 9}$ & $\%$ Change & $\mathbf{2 0 0 8}$ & $\mathbf{2 0 0 9}$ & $\%$ Change \\
\hline SOV & 1061 & 3778 & $256.1 \%$ & 9141 & 12206 & $33.5 \%$ \\
\hline HOV2 & 3040 & 1899 & $-37.5 \%$ & 10437 & 8181 & $-21.6 \%$ \\
\hline HOV3 & 477 & 171 & $-64.2 \%$ & 2335 & 2558 & $9.6 \%$ \\
\hline Transit & 810 & 821 & $1.4 \%$ & 810 & 821 & $1.4 \%$ \\
\hline Total & 5387 & 6669 & $23.8 \%$ & 22723 & 23766 & $4.6 \%$ \\
\hline
\end{tabular}

\section{Findings from South Florida Region Commuter Survey (May 2009)}

\section{Express Lane Users}

To further investigate these mode shifts, the survey data from a May 2009 survey was analyzed. In May 2009 a survey was distributed to commuters in the South Florida Region to gauge feedback on the I-95 Express Lanes (Phase 1A). The survey data was provided by Jim Udvardy from South Florida Commuter Services. To begin, an analysis of mode shifts among survey respondents was examined. Table 5 and Table 6 show the mode used by Express lane users and their modes before Express Lanes (prior mode) for commuters and non-commuters respectively. 
(Note the percentages in the table don't sum up to 100 percent for each mode in Express Lanes because respondents could select more than one prior mode).

Table 5 Mode Used to Access the Express Lanes and Corresponding Prior Modes (commuters)

\begin{tabular}{|l|c|c|c|c|}
\hline & \multicolumn{3}{|c|}{ Prior Mode } & \multirow{2}{*}{ Total } \\
\cline { 1 - 4 } $\begin{array}{l}\text { Current Mode to access } \\
\text { Express Lanes }\end{array}$ & $\begin{array}{c}\text { Carpool in } \\
\text { HOV lanes }\end{array}$ & $\begin{array}{c}\text { Hybrids in HOV } \\
\text { lanes }\end{array}$ & $\begin{array}{c}\text { Not Carpool } \\
\text { Lanes }\end{array}$ & \\
\hline Toll users (SOV/HOV2) & $13 \%$ & $1 \%$ & $83 \%$ & 2693 \\
\hline Registered HOV3+ & $86 \%$ & $4 \%$ & $11 \%$ & 56 \\
\hline Vanpool & $80 \%$ & $5 \%$ & $20 \%$ & 20 \\
\hline Hybrid & $6 \%$ & $75 \%$ & $21 \%$ & 134 \\
\hline
\end{tabular}

Table 6 Mode Used to Access the Express Lanes and Corresponding Prior Modes (noncommuters)

\begin{tabular}{|l|c|c|c|c|}
\hline & \multicolumn{3}{|c|}{ Prior Mode } & \multirow{2}{*}{ Total } \\
\cline { 1 - 4 } $\begin{array}{l}\text { Current Mode to access } \\
\text { Express Lanes }\end{array}$ & $\begin{array}{c}\text { Carpool in HOV } \\
\text { lanes }\end{array}$ & $\begin{array}{c}\text { Hybrids in HOV } \\
\text { lanes }\end{array}$ & $\begin{array}{c}\text { Not Carpool } \\
\text { Lanes }\end{array}$ & 2989 \\
\hline Toll users (SOV/HOV2) & $18 \%$ & $2 \%$ & $75 \%$ & 2989 \\
\hline Hybrid & $2 \%$ & $86 \%$ & $14 \%$ & 169 \\
\hline
\end{tabular}

Most of the toll paying users of the Express Lanes came from GPLs (irrespective of mode) among both the commuters ( 83 percent) as well as the non-commuters ( 75 percent). However, there is also an indication that some previous carpoolers in the HOV lanes becoming toll users in the Express Lanes (13 percent and 18 percent in commuters and non-commuters respectively). Since the toll users also include HOV2s, whether the carpools broke up to become SOVs is not clear from the above data, but will be examined further in the next section.

\section{Usual Mode, Prior Mode and Mode to Access Express Lanes}

For this analysis, the 'usual mode' of respondents was defined as the mode used by them for at least three days based on the question asked in the survey -"How do you usually get to and from work/school? Select the mode you use most often (3 or more days per week)." For the analysis only those respondents who mentioned they have used the Express Lanes were examined. To investigate the relationship between the usual mode of travelers and their prior mode, as well as their mode to access Express Lanes, respondents were grouped based on their usual modes (see Table 7 and Table 8). 
Table 7 Usual Mode of Express Lane Users and Their Prior Mode

\begin{tabular}{|l|c|c|c|}
\hline \multirow{2}{*}{$\begin{array}{c}\text { Usual Mode } \\
\text { (No. of respondents) }\end{array}$} & \multicolumn{3}{|c|}{ Prior Mode } \\
\cline { 2 - 4 } SOV (2558) & Carpool in HOV & Hybrid in HOV & Not Carpool lanes \\
\hline HOV2 (158) & $71 \%$ & $4 \%$ & $86 \%$ \\
\hline HOV3+ (120) & $81 \%$ & $4 \%$ & $24 \%$ \\
\hline
\end{tabular}

Note: The percentages for each mode don't sum to 100 percent since the respondents could select more than one mode.

Table 8 Usual Mode of Express Lane Users and Their Mode to Access Express Lanes

\begin{tabular}{|l|c|c|c|c|}
\hline \multirow{2}{*}{ Usual Mode } & \multicolumn{4}{|c|}{ Mode to Access Express Lanes } \\
\cline { 2 - 5 } & Toll Paying (SOV/HOV2) & Registered HOV3+ & Hybrid & Vanpool \\
\hline SOV & $94 \%$ & $0 \%$ & $5 \%$ & $0 \%$ \\
\hline HOV2 & $92 \%$ & $3 \%$ & $6 \%$ & $0 \%$ \\
\hline HOV3+ & $61 \%$ & $33 \%$ & $1 \%$ & $16 \%$ \\
\hline
\end{tabular}

Note: The percentages for each mode don't sum to 100 percent since the respondents could select more than one mode.

Almost all the Express lane users who mentioned SOV and HOV2 as their usual modes are toll paying users of Express lane (94 percent and 92 percent respectively in Table 8). In the case of HOV2s, a quarter of them (Table 7) were not using HOV lanes prior to Express Lanes when HOV lanes had 2+ carpool requirement and unrestricted access points. Now, almost all of them (92 percent in second table) use the Express Lanes as paying users. This is a clear indication of the improved travel conditions on the Express Lanes.

\section{Breaking-up of the Carpoolers (HOV3+ Usual Mode)}

Table 7 shows that those who have HOV3+ as their usual mode, 81 percent of them previously used the HOV lanes as carpools. However, with Express Lanes, only 33 percent (Table 8) are the registered HOV3+ users while 61 percent are toll paying LOVs (SOV/HOV2+). This shift of the HOVs to toll paying LOVs can be attributed to the strict registration requirement for the carpool registration because of which lesser number of HOV3+ travelers use the Express lanes as registered carpools. 


\section{Reasons for Using the Express Lanes}

Respondents who have used the Express Lanes were asked the reasons for using the lanes. The respondents were divided into the modes they used to access Express Lanes (see Table 9).

Table 9 Reasons for Using the Express Lanes

\begin{tabular}{|l|c|c|c|}
\hline \multirow{2}{*}{ Why do use the Express Lanes } & \multicolumn{3}{c|}{ Mode to access Express Lanes } \\
\cline { 2 - 4 } & Carpool & $\begin{array}{c}\text { Toll Paying } \\
\text { (HOV2/SOVs) }\end{array}$ & Hybrids \\
\hline Saves time & $79 \%$ & $77 \%$ & $81 \%$ \\
\hline Moves faster than general lanes & $89 \%$ & $77 \%$ & $83 \%$ \\
\hline Less crowded & $70 \%$ & $72 \%$ & $78 \%$ \\
\hline Cost savings & $32 \%$ & $5 \%$ & $15 \%$ \\
\hline $\begin{array}{l}\text { Trucks and larger vehicles are not in the } \\
\text { lanes }\end{array}$ & $36 \%$ & $33 \%$ & $43 \%$ \\
\hline I can use the lanes toll free & $54 \%$ & $2 \%$ & $85 \%$ \\
\hline I feel safer in the lanes & $18 \%$ & $18 \%$ & $24 \%$ \\
\hline My employer pays the cost of tolls & $0 \%$ & $3 \%$ & $1 \%$ \\
\hline
\end{tabular}

More than three-quarters of the respondents in each mode indicated the time-savings/ freeflowing traffic factors of the Express Lanes as the reasons for using them. Apart for these, the absence of trucks and safety perception in the lanes are also important reasons for using the Express Lanes. Absence of trucks is indicated by at least one-third respondents in each mode. In addition a perception of safety is indicated by 18 percent of both the carpool and toll paying users while close to a quarter of the Hybrid respondents indicated that.

\section{Express Lane Non-Users}

The following section focuses on those respondents who indicated they do not use the Express Lanes but previously used the carpool lanes. This group of respondents is important in providing an insight in to the reasons for which they do not use the HOV lanes after the conversion.

\section{Prior Mode in HOV Lanes}

The prior mode used while traveling in the HOV lanes for those respondents who indicated they do not use the Express Lanes anymore is shown in Table 10. The total number of respondents was 262. Almost three-quarters of the respondents were previously carpooling in HOV lanes and more than one-tenth were Hybrids. Since these respondents (most of them carpoolers) do not use the Express Lanes while they used the HOV lanes previously, these findings again support the 
decrease in the number of HOV2 and HOV3+ in 2009 after the Express Lanes started as shown in Table 4.

Table 10 Percentage of Respondents by Their Prior Mode in HOV Lanes

\begin{tabular}{|l|c|}
\hline Prior mode in HOV lanes & Percentage of respondents \\
\hline Carpool & $73.7 \%$ \\
\hline Hybrid & $13.4 \%$ \\
\hline Motorcycle & $4.2 \%$ \\
\hline Special Privileges & $8.8 \%$ \\
\hline
\end{tabular}

\section{Reasons for Not Using the Express Lanes by Previous Carpoolers in HOV Lanes}

Respondents were also asked the reasons for not using the Express Lanes. Table 11 shows the reasons by those who were previously carpooling in the HOV lanes. The total number of respondents was 180 (percentages don't sum to 100 percent because respondents could select more than one reason). The majority of respondents (52 percent) selected 'not willing to pay a toll' as the reason for not using the express lanes followed by more than one-third respondents each for 'the express lanes are too expensive', and 'no access to get in to or out of lanes where they get on I-95'. Since toll is mentioned by the majority of the respondents as one of the reasons for not using the Express Lanes, it might also indicate that most carpoolers were HOV2s and not HOV3+ since not qualifying as a $3+$ carpool is mentioned by only one-tenth of the respondents.

\section{Table 11 Reasons for Not Using the Express Lanes by Previous Carpoolers in HOV Lanes}

\begin{tabular}{|l|c|}
\hline Why do you not use the Express Lanes? & Percentage of respondents \\
\hline My carpool does not qualify as a 3+ carpool. & $11.1 \%$ \\
\hline I prefer to use the general lanes. & $10.0 \%$ \\
\hline I do not want to pay a toll. & $51.7 \%$ \\
\hline It is too expensive. & $34.4 \%$ \\
\hline There is no access to get into the lanes where I get on I-95 & $36.7 \%$ \\
\hline There is no access to get out of the lanes where I get on I-95. & $33.9 \%$ \\
\hline
\end{tabular}

\section{Reasons for Not Using the Express Lanes by Previous Hybrid Users in HOV Lanes} There were only 35 respondents among those who mentioned hybrid vehicles as their mode to access HOV lanes. A majority of them (20 out of 35) mentioned access inconvenience as one of the reasons for not using the Express Lanes. 
These results indicate how, the changes in the design and operations of new Express Lanes affect the usage of Express Lanes by the former users of the HOV lanes. I-95 corridor, therefore, gives an opportunity to better understand the different effects Express Lanes implementation can have on the previous users of the HOV lanes due to the many operational and design modifications to the previous HOV lanes.

Among the changes made in the design and operations of the Express Lanes, access and toll are the two major reasons for carpoolers not using the Express Lanes. Not qualifying as a 3+ carpool was also mentioned, but only by 11 percent of the respondents. Although, this may have been because of the small percentage of $\mathrm{HOV} 3+$ respondents among the carpoolers.

\section{Safety Implications of the Express Lanes}

In the May 2009 survey of South Florida commuters (SFCS, 2009), there were 169 comments from the respondents on the design of Express Lanes complaining about the narrow lanes being unsafe, cars driving over the plastic poles to access the Express Lanes, and suggesting a barrier separation. In addition to this, there were 110 responses complaining about the safety of the express lanes with people cutting in and out of the Express Lanes and no shoulder to pull over in case of emergency. (Note that Express Lanes were converted from one HOV lane to two Express Lanes and during this process the shoulder was reduced and lanes were narrowed to 11 feet).

In the May 2009 survey, when respondents were asked how the safety of Express Lanes changed after the conversion, 22 percent of the respondents mentioned the lanes have becomes unsafe, 33 percent mentioned safer and remainder were neutral. On the other hand, the perception of increased safety was indicated by the respondents as one of the reasons for using the Express Lanes. When Express lane users were asked the reasons for using the Express Lanes- 18 percent of both the carpool and toll paying users mentioned "I feel safer in the lanes" while close to a quarter of the hybrid users indicated this.

According to Florida DOT's mid-year evaluation report (FDOT, 2009) “95 Express [Lanes] has not been in operation long enough to collect any significant crash data. However, day to day monitoring of the facility and evaluations of incidents to date has not indicated any safety 
concerns." However no information was available regarding the violations in terms of cars driving over the poles to access Express Lanes.

As part of 95 Express Lanes operations, off-duty FHP officers are also contracted to provide additional visual enforcement within the Express Lanes (FDOT, 2009). However, as observed from the survey findings, the effectiveness of the enforcement efforts is not yet measurable. Therefore, the I-95 Express Lanes have not yet achieved their objective of increasing safety.

\section{Throughput}

Comparing 2008 and 2009, the person throughput during the PM peak hour (4 PM-5 PM) in HOV/HOT lanes and GPLs increased by 23 percent and 8 percent respectively. The person throughput in Express Lanes increased even when the average vehicle occupancy dropped from 1.95 (2008) to 1.39 (2009) due to SOVs being allowed in Express Lanes. Overall, the person throughput increased by 1,325 or 12 percent in the facility after the Express Lanes implementation (FDOT, 2009). It should be noted that there was an addition of one more lane in the northbound direction.

During the first six months of operations, on average, during the PM peak period (4 PM to 7 PM) the Express Lanes carried 27.7 percent of the total traffic on the corridor (6,910 in Express Lanes and 18,064 in GPLs) with 33 percent of the total capacity (2 Express Lanes and 4 GPLs) (FDOT, 2009).

\section{Travel Time Reliability}

In the first six months of Express lane operations, the Express Lanes considerably improved the overall operational performance of I-95. The travel speed during PM peak periods (4 PM- 7 PM) significantly increased from an average speed in the HOV lane of approximately $20 \mathrm{mph}$ to an average of $57 \mathrm{mph}$. The speed in the GPLs has also increased from an average of approximately $20 \mathrm{mph}$ to an average of $41 \mathrm{mph}$. Average volume along the Express Lanes in the PM peak period (4 PM to 7 PM) was nearly 7,000 vehicles (approximately 28 percent of the total I-95 northbound traffic). After one year of the Express Lanes operations in December 2009, Express Lanes operated at a speed of $45 \mathrm{mph}$ or greater for 99.3 percent of the time (FDOT, 2009). 


\section{Ridesharing and Transit}

\section{Transit}

Due to the Express Lanes, the travel time of buses decreased from 25 minutes to 8 minutes and the travel time reliability increased. The bus ridership also increased by 30 percent as compared to the year before Express Lanes. After one year of operation in December 2009, buses (Miami Dade Transit and Miami Dade School) represented 36 percent (2782 buses) of the total toll exempt registration (7801).

\section{HOV3+ and Hybrids}

The total number of HOV3+ registrations increased from 1356 in first six months to 1705 after one year (22 percent of total toll exempt vehicles). The number of Hybrid registrations also increased from 2891 to 3264 during this period (FDOT District Six, 2010 and FDOT, 2009) and have the highest share (42 percent after one year) among all the toll exempt registered vehicles. Therefore, the highest proportion of monthly toll exempt trips is by Hybrids only (67 percent of total toll exempt monthly trips averaged over first six months) (FDOT, 2009).

In December 2009, after one year of operation, toll exempt trips are 1.3 percent of the total trips on Express Lanes in a month. The low usage of the Express Lanes by toll exempt vehicles can be seen from the fact that the average monthly trips by toll exempt vehicles are almost equal to the total number of toll exempt vehicles. For example, in December 2009, after one year of the Express Lanes opened, there were 7801 registered vehicles and 8813 toll exempt vehicle trips for the month.

The objectives of the I-95 Express Lanes were: improving the safety, throughput, and reliability of the corridor and encouraging the ridesharing and transit use in the corridor. Based on the first years of operations the Express Lanes have been successful in increasing the throughput and reliability of travel, plus transit ridership has also increased. However, the objective of improved safety has not yet been achieved. There are increased safety concerns among Express Lanes users, mostly due to the modified design (narrower lanes, no side shoulder and flexible poles). Also, in terms of toll exempt trips, the Hybrid registrations are almost double that of HOV3+ registrations and the frequency of toll exempt trips is also very low. This indicates that by 
increasing the occupancy requirement for free carpool access to 3+ along with strict registration requirements, I-95 Express Lanes have not been able to achieve the objective of encouraging ride sharing and $\mathrm{HOV} 3+$ ridesharing forms a small proportion of toll exempt vehicles.

\section{SR 91 Express Lanes and I-95 Express Lanes}

\section{Similarities between the Express Lanes}

There are many similarities between the SR 91 Express Lanes (December 1995 through January 1998, the period when HOV3+ could access the Express Lanes for free) and I-95 Express Lanes Similarities include both operation as well as design of the Express Lanes. Both the corridors have:

- two Express Lanes and four GPLs in each direction,

- HOV3+ requirement for free carpool access to the Express Lanes,

- no intermediate access to the Express Lanes, and

- flexible pylons separating the GPLs and the Express Lanes with no median shoulder.

Both corridors had high congestion levels before the Express Lanes. Around the time of Express lane implementation, ADT on the two corridors was 198,563 vpd for SR 91 and 290,000 vpd for I-95 before the Express Lanes started. Major differences include- there was no HOV lane in the SR 91 corridor and the SR 91 express bus route 149 does not use the Express Lanes because of most of that route's ridership is from local stops leading to difficulty in routing the bus on to the freeway. Despite these two differences, the many similarities between the two Express Lanes provide an opportunity to compare the different impacts Express Lanes implementation had in their respective corridors.

\section{Impact on $\mathrm{HOV} 3+$}

After one year of operation of the SR 91 Express Lanes, there was an increase of 40 percent of HOV3+ vehicles in the peak period (496 to 725) while there was an increase of around 10 percent (778 to 852) of HOV3+ vehicles on I-95 Express Lanes. However the large difference in the percentage increase may be largely due to low number of baseline HOV3+ in SR 91 corridor since, in SR 91, there were previously no HOV lanes and less incentive for carpooling (Sullivan, 1998). Also, the increase in HOV3+ in SR 91 can be attributed primarily to the implementation 
of Express Lanes. In case of I-95 there was 64 percent reduction of HOV3s in the Express Lanes and an increase in HOV3s in GPLs.

Next the usage of the Express Lanes by the toll exempt vehicles in the two corridors after one year of operations (December 1996 in SR 91 and December 2009 in I-95) were compared. One way, weekday volume of HOV3+ in SR 91Express Lanes (2500 vehicles) is approximately equal to the 30 percent of the total toll exempt trips (including Hybrids, Buses, motorcycles) in one month (8813 trips) in I-95 Express Lanes.

In terms of frequency of usage, nearly 85 percent of HOV-3+ Express lane users use the SR 91 Express Lanes for more than 60 percent of their work trips, and most of these use the toll lanes at least 90 percent of the time (. On the other hand, the low usage frequency of the Express Lanes by $\mathrm{HOV} 3+$ in I-95 is reflected from the fact that after one year of Express Lanes, total monthly toll exempt trips (8813) were almost equal to total toll exempt registered vehicles (7801).

There are different factors which could be responsible for the different HOV3+ usage trends in the two corridors-

- Induced traffic: Over the first year of Express lane operations on SR 91, there was an increase of 14 percent (28000 vehicles) in the ADT of the corridor. And 60 percent of that increase was induced traffic (other increase in traffic included the long term growth of traffic and traffic from parallel city streets). According to one estimate (Sullivan, 1998), during the PM peak period, this induced traffic contributed to 39 percent increase in the pre-Express level of HOV3+. On the other hand, over the six months, northbound traffic on I-95 (Express and GPLs) decreased by almost 3 percent.

- New incentives for carpooling: Unlike on the I-95 corridor, there were no HOV lanes on the SR 91 corridor. With Express Lanes, SR 91 travelers had an additional incentive for $3+$ carpooling on the corridor. This also explains the high percentage increase in HOV3+ volume on the SR 91 corridor.

- Registration Requirement: On SR 91Express Lanes, registration is required by all users (toll and non-toll) with no extra eligibility requirements for carpool members other than the number of people in the vehicle. However, on the I-95 Express Lanes, along with 
registration, there are strict guidelines (constraints of work and home vicinity of the participants) to register as a carpool. In the I-95 survey, 66 percent of those whose typical mode is HOV3+ use the HOT lanes as toll users.

- Express lane availability: SR 91 Express Lanes started operating in both the directions from the day they began operations. The I-95 Express Lanes began only in one direction (northbound, going away from downtown Miami). In the southbound direction, the HOV lanes were still congested during peak periods. Therefore, for the two-way commuters, there might not have been enough incentives to register as a carpool and use the lanes or switch to transit.

- Access Convenience: SR91 survey responses had almost no complaints about a lack of intermediate access points. This was not surprising given the commuter patterns of many people using the facility. However in a fall 1996 telephone survey of SR 91 commuters it was found that 76 percent of the HOV3+ used the Express lanes for their trip while 24 percent did not. Mastako et al. (1998) attributed the reason for not all the HOV3+ using the Express lanes (even though they could use the lanes for free) to the access inconvenience. In the I-95 survey, almost one-third of the respondents who previously carpooled in HOV lanes but do not use the Express Lanes mentioned access inconvenience as one of the reasons. This difference in the perception of access inconvenience between the two Express Lanes may be because I-95 carpoolers were already used to unrestricted access to the HOV lanes while SR 91 travelers had no HOV lanes and hence, SR 91 Express Lanes was a fresh start for them.

- Availability of express bus on Express Lanes: There was no transit option available on the SR 91 Express Lanes while the I-95 Express Lanes had more than 900 registered buses using the Express Lanes. Therefore, there is a possibility that some potential HOV3+ users may have shifted to transit (or increased their frequency of using it). This is also supported by looking at the availability of vehicles for transit users. In a 2009 survey of transit users on I-95, more than 60 percent of the respondents had a personal vehicle available for their trip. This is an example in which availability of alternative modes (transit in this case) to travelers in Express lane corridors leads to different impacts by the same policy. In this case, HOV3+ eligibility for the free access to Express Lanes had different impacts on ridership patterns in the two corridors. 
This also underscores the necessity of evaluating the mode shift behavior of transit users by conducting user surveys. A survey of transit users was conducted (Cain, 2009) to evaluate the impacts of I-95 Express lane implementation. Unfortunately there were not enough respondents who joined the transit after the Express Lanes started and therefore no conclusions could me regarding their mode shift.

\section{Impact on HOV2}

Since both SR 91 and I-95 Express Lanes treat HOV2s as toll paying vehicles similar to SOVs, their impact on HOV2s can be examined and compared. However, as was the case when examining impact of the Express Lanes on HOV3+ travel, the I-95 Express Lanes were preceded by a HOV lane already encouraging carpools in that corridor.

After the SR 91 Express Lanes opened, there was a significant increase in HOV3+ vehicles as well as SOVs in the corridor. However, only a small increase in the number of HOV2s was observed. Only 30 percent of HOV2s used the SR 91 Express Lanes as compared to 60 percent in conventional HOV lanes in study areas during the peak 2 hours. Therefore, "opening of the SR 91 Express Lanes did relatively little to encourage or discourage HOV-2 ridesharing" (Sullivan, 1998).

Looking at the 2 hour PM peak traffic on the I-95 corridor, HOV2s decreased by 38 percent in the HOV lanes and 15 percent on the GPLs. There are findings from the survey which explain the decrease in HOV2 volume in the Express Lanes. More than 50 percent of the carpoolers (occupancy not known) who previously traveled on HOV lanes mentioned "they do not want to pay a toll" as one of the reasons for not using the Express as opposed to 10 percent mentioning "not eligible for 3 + carpool."

Only 23 percent of all HOV2s in the corridor use the I-95 Express Lanes during the 2 hour PM peak period (30 percent in case of SR 91). Therefore, the percentage of HOV2s using the Express Lanes on both corridors is clearly less than those in conventional HOV lanes (60 percent). This may be because the only incentive left for HOV2 as compared to SOV mode on 
Express lane is that they could split the tolls. The above findings indicate that with paying HOV2s on Express Lanes, there would be reduced encouragement for 2 person carpools.

\section{Impact on Transit}

When bus riders on the SR 91 corridor in late 1996 were asked if the toll lanes have changed their travel in any way- only 14 percent answered positively and 60 percent said no. When bus riders along I-95 were asked their perception regarding different elements of transit as compared to pre Express lane implementation in May 2009, a majority of the respondents mentioned service reliability ( 55 percent) and travel time ( 75 percent) are better with the new Express Lanes. This is because the express bus on SR 91 corridor does not use the Express Lanes while express bus on I-95 does. With Express Lanes in I-95 the travel time of buses was reduced from 25 minutes to 8 minutes. However, there were no evidences of SR 91 Express Lanes changing bus operation in any way. After the Express Lanes there was no change in ridership for the express bus on SR 91 but there was a 30 percent increase in ridership for the express bus in I-95.

\section{Impact on Safety}

Both the Express Lanes have two Express Lanes in each direction with no median shoulder and plastic pylons within the buffer to separate the Express Lanes from the GPLs. In case of I-95, the Express Lanes are narrower (11 feet wide). The accident rate for the section of SR 91 containing the express lanes decreased significantly after the express lanes opened. When respondents were asked the reasons for using the express lanes other than travel time savings, 40 percent mentioned driving comfort and safety of the SR 91 Express Lanes. In case of SR 91, there was no issue of people cutting in and out of the Express Lanes was mentioned. Also, there were almost no comments from survey respondents indicating any of those issues.

In the I-95 survey when respondents were asked how the safety of Express Lanes changes after the conversion, 22 percent respondents mentioned the lanes have becomes unsafe, 33 percent mentioned safer and rests were neutral. Also, from the survey there were many comments on the inadequate design of Express Lanes (narrower lanes and no shoulder) and people cutting in and out of the lanes because of plastic poles. At the same time, some survey respondents mentioned safety as one of the reasons for using the Express Lanes. Among the reasons mentioned for using 
the Express Lanes, absence of trucks is indicated by at least one-third respondents in each mode followed by safety perception which was indicated by 18 percent of both the carpool and toll paying users while close to a quarter of the Hybrid users mentioned this.

\section{Usage of Express Lanes}

After one year of Express lane operations in SR 91 (December 1996) and in I-95 (December 2009), one way weekday traffic in SR 91 Express Lanes reached around 13,000 to 14,000 while in I-95 Express Lanes it reached more than 23,000. It should be noted that the ADT of the two corridors at the time of Express Lanes implementation was 202,400 and 290,000 respectively. Assuming one-way ADT of the SR 91 corridor to be half of the total ADT $(226,600)$ the two Express Lanes carried 11 percent and 15 percent of the total one-way ADT of the corridor respectively.

There is a significant difference between the frequency of usage of Express Lanes by toll exempt trips in I-95 and HOV3+ in SR 91. In I-95 Express Lanes, the low frequency of toll exempt vehicles can be seen from the fact that after one year of operation the total number of toll exempt trips in December 2009 (8813) was almost equal to the total toll exempt registered vehicles (7801). On the other hand, in SR 91 Express Lanes, about 75 percent of HOV-3+ work commuters were found to be frequent express lane users, defined as using the lanes for at least 60 percent of their work trips. This compares to 26 percent and 16 percent, respectively, of HOV-2 and SOV commuters.

After one year of operations of Express Lanes, in terms of numbers, monthly toll exempt trips on I-95 Express Lanes are 1.3 percent $(8813$ out of 648,430) of the total trips on Express Lanes. Comparing it to the SR 91 Express Lanes, in December 1996 (after one year of operation), the number of HOV3+ vehicles in one day in one direction (2500) was almost 30 percent of the total monthly toll exempt trips on I-95 (8813). Note that HOV3+ form less than a quarter of the total toll exempt vehicles on I-95. Therefore, in terms of usage of Express Lanes by 3+ carpools, SR 91 Express Lanes performed far better than the I-95 Express Lanes. 
However, it should also be noted that on SR 91 Express Lanes, there were no express buses while in I-95, there are 900 Miami Date Transit buses and 1700 Miami Dade school buses using the Express Lanes. The presence of Express bus service on I-95 can also be one of the reasons for lesser usage of Express Lanes by HOV3+. Some of the potential 3+ carpools on I-95 might have started to use the Express bus in order to avoid strict registration requirement. However, from the survey of transit users, it was found that those 50 percent of those who shifted to transit after the Express Lanes started (27 out of 50 respondents) previously drove alone and none of them carpooled. Due to small number of respondents researchers made no conclusions regarding this mode shift. 


\section{CHAPTER IV}

\section{I-15 (FROM 1996 UNTIL 2008), SAN DIEGO AND I-25 EXPRESS LANES, DENVER}

This chapter examines two HOT lanes-- I-15 Express Lanes near San Diego and I-25 Express Lanes near Denver. These were chosen as a pair due to their many similarities.

\section{I-15 Express Lanes: Introduction}

The I-15 Express Lanes in San Diego extended 8 miles in the median of I-15, from SR 163 to SR 56/Ted Williams Parkway (see Figure 7). The Express Lanes are called the I-15 Express Lanes. It was a two-lane, reversible facility with access available only at its two ends. SOVs can pay a fee to use the lanes. HOVs of two or more occupants (carpools, vanpools, and buses), motorcycles, designated hybrid vehicles and two-axle trucks are permitted to use the I-15 Express Lanes for free.

In September 2008 a new, 4.5 mile, four-lane segment of Express Lanes extending north from SR 56/Ted Williams Parkway to Rancho Bernardo Road opened (see Figure 7). This research will focus on the operations of Express Lanes from 1996 until August 2008. 


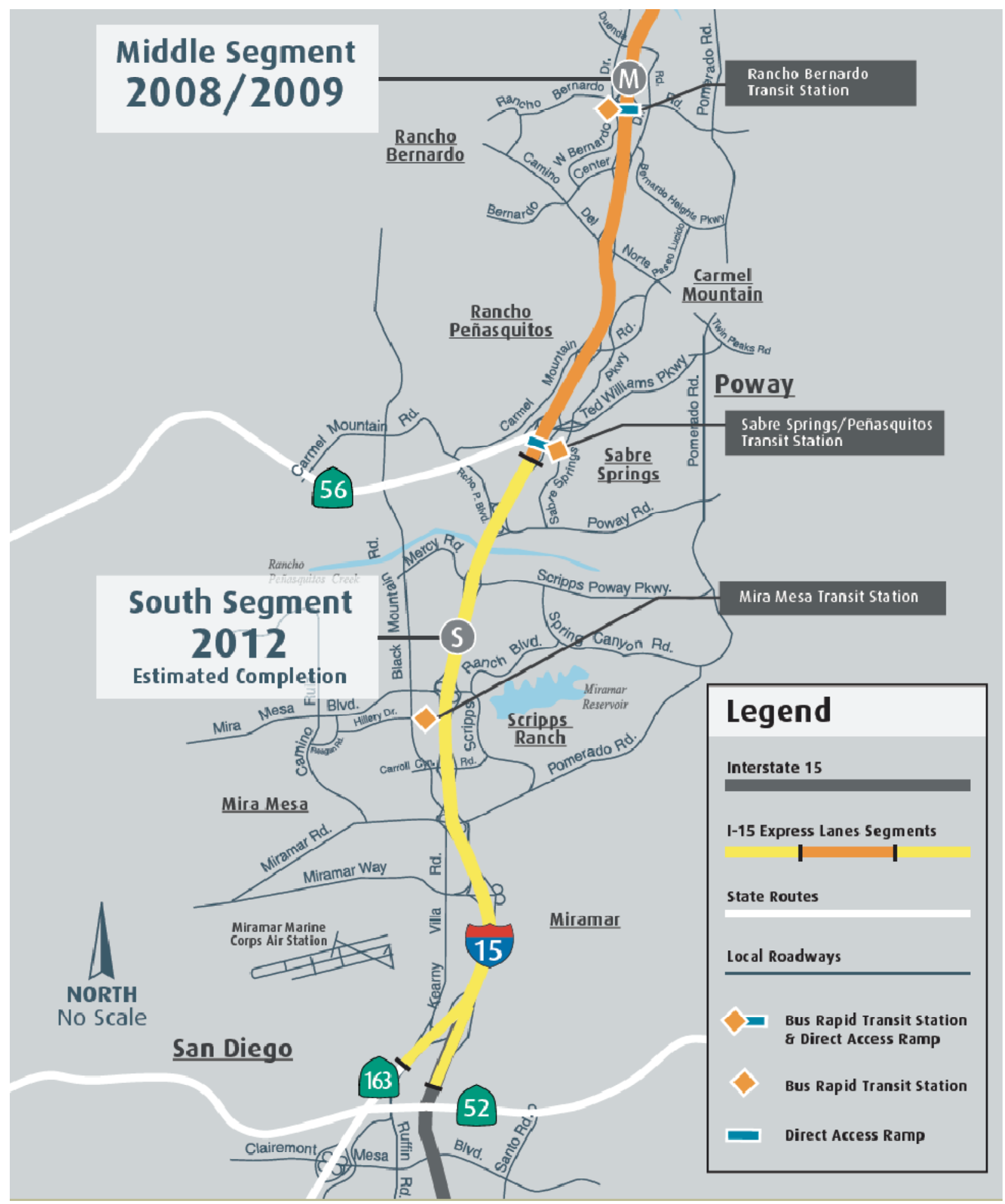

Figure 7 I-15 Corridor Map Showing Original 8 Mile Section (SR 163 to SR 56/Ted Williams Parkway) and New 4.5 Mile Section (SR 56/Ted Williams Parkway to Rancho Bernardo Road)(Source : www.keepsandiegomoving.com ) 


\section{Timeline of the I-15 San Diego Express Lanes}

The Express Lanes started in December 1996 with Phase-I Express Pass program in which a limited number of solo drivers paid a flat fee for unlimited use of the I-15 Express Lanes. Initially, 500 monthly ExpressPass permits were made available in December 1996 at a cost of $\$ 50$ per month. At the end of Phase I, in March 1998, a total of 1,000 ExpressPass permits were available at $\$ 70$ per month.

In late March 1998, the program began Phase II I-15 FasTrak, which allowed solo drivers to pay a per-trip fee to use the lanes. The fees were adjusted dynamically on the basis of traffic levels in the I-15 Express Lanes as well as time of day. On August 31, 1998, this dynamic toll pricing was modified to reduce maximum tolls in off-peak periods to encourage greater FasTrak usage outside of the peak hours.

To date, these fees have varied between $\$ 0.50$ and $\$ 4.00$ per trip; during more severe traffic conditions in the Express Lanes, they can go up to $\$ 8.00$ per trip. A Level of Service (LOS) C or better is required by law to be maintained on the Express Lanes at all times.

Initially the I-15 Express Lanes operated in the southbound direction (inbound commute) from 6:00 AM to 9:00 AM and in the northbound direction (outbound commute) from 3:00 PM to 6:30PM. The hours of operation were extended in November 1997 from 5:45 to 9:15 AM and from 3:00 to 7:00 PM. The facility is closed on weekends and holidays (Supernak et al., 2002).

\section{Previous HOV Lanes}

I-15 consists of the four GPLs in each direction over the segment with HOV lanes with emergency lanes on either side of the traffic lanes. Some widening occurs in the transition areas to and from SR 163. The GPLs were separated from the HOV lanes by a fixed barrier wall in the narrow median areas of the project. There was no intermediate access between the GPLs and the HOV lanes other than the end points. With Express Lanes there was no change in the separation mechanism and access points (Wilbur Smith, 1997). 


\section{Traffic Conditions on the Corridor Before the Express Lanes}

\section{Peak Hour Volume}

Based on the traffic data collected by Caltrans and Wilbur Smith Associates (WSA) during October 1996 before the Express Lanes started, the average weekday volume for the HOV lanes was 9600 vehicles (Wilbur Smith, 1996). During the peak hour, the average weekday volume in two HOV lanes varied from 1794 to $2126 \mathrm{vph}$. Based on WSA's analysis of the critical 1-15 HOV lane segments, there was a reserve capacity ranging from 500 to 1,100 vehicles per hour which could be allocated for the SOV "buy-in" into the HOV lanes during the peak hour. Peak hour traffic flow for I-15 GPLs (at Carroll Canyon Road) was approximately 11,900 vehicles southbound during the morning and 10,100 vehicles northbound in the evening.

\section{Travel Times}

Morning peak period (6:00 to 9:00 AM): During the morning peak period, travel times in GPLs for the approximately 7.5-mile distance ranged from 7.1 minutes to 11.9 minutes while it ranged from 6.3 minutes to 7.2 minutes for the 7.7 mile distance on HOV lanes.

Evening peak period (3:00 to 6:30 PM): During the evening peak period, travel times for the mainline lanes ranged from 7.2 minutes to 14.4 minutes while it ranged from 6.4 minutes to 7.3 minutes on the HOV lanes.

\section{Why Were the Express Lanes Considered?}

In the early 1990s, the HOV lanes were underutilized; at the same time, the GPLs experienced severe congestion and transit service in the corridor was limited. In 1991, San Diego Association of Governments (SANDAG) expressed concern about the relative lack of transit in the I-15 corridor, the underutilization of the HOV lanes, and congestion on the GPLs. A resolution was adopted in May 1991 supporting a demonstration project to test the feasibility of pricing the use of the I-15 Express Lanes by SOVs, with revenues allocated to increase transit in the I-15 corridor.

A state law was passed in October 1994 requiring that the I-15 Express Lanes ensure free-flow conditions for HOVs at all times. Free-flow conditions constitute level-of-service (LOS) B or the 
preexisting level of service, which the California Department of Transportation (Caltrans) determined to be LOS C. According to the law, unrestricted, free access to the lanes by HOVs is to be available at all times. In addition, the law limits the use of revenue to transit and HOV improvements for the I-15 corridor (Supernal et al., 2002).

The project's primary goals were:

- To maximize use of the existing I-15 Express Lanes,

- To test whether allowing solo drivers to use the Express Lanes' excess capacity could help relieve congestion on the I-15 main lanes,

- To fund new transit and HOV improvements in the I-15 corridor, and

- $\quad$ To use a market-based approach to set tolls (Supernak et al., 2002).

\section{Impact on Transit}

One of the goals of the I-15 Congestion Pricing Project was to expand transit services. As part of the project, San Diego Metropolitan Transit Development Board (MTDB) developed a service plan for new I-15 transit service. In March 1997, SANDAG allocated \$1.8 million of fee revenue for a new I-15 express bus service called the Inland Breeze (Route 990). The new service, which began in November 1997, used the I-15 HOV lanes in the peak periods and the mixed-flow lanes during the off-peak. There were four other routes which used the HOV lanes before and after the Express lane conversion (SANDAG, 1999).

Several studies were led by San Diego State University researchers before and after the introduction of SOVs to the Express Lanes. During the period of the studies, the ridership of the Inland Breeze grew from 188 riders on the first day to a maximum of 598 on February 21, 1999; however, the goal of 750 riders was not met in the first two years of service (November 1997 to November 1999). On the last day of April 1999, the Inland Breeze route carried 712 riders

Based on passenger counts and surveys collected on the Inland Breeze route, researchers determined that most riders (75 percent) were commuting in the reverse direction-away from downtown in the morning, and towards downtown in the afternoon. This was consistent with ridership trends for other routes in the same corridor. In addition, most survey respondents were 
captive riders - they did not have a car available and rode other transit routes before the Inland Breeze service was introduced. The majority of respondents were also not familiar with FasTrak, the toll program required for SOV users to travel on the Express Lanes. (Kaschade et al. 2001)

The intention in funding the Inland Breeze express bus service with FasTrak revenue was to provide access to I-15 Express Lanes to the commuters traveling in the peak commute direction in the heavily congested I-15 corridor. However, the strong reverse commute ridership using the GPLs was not fulfilling the main intent of the service. To increase ridership levels, particularly in the peak commute direction a revised service plan was approved. Under this plan, starting January 2000, the Inland Breeze operated as a direct commuter express service between Rancho Bernardo/Poway to downtown San Diego, traveling southbound in the AM peak period and then northbound from downtown in the PM peak period. In the reverse commute direction (i.e., northbound in the AM and southbound in the PM), the route continued to make limited stops in Hillcrest, Fashion Valley Transit Center, Kearny Mesa, Mira Mesa, and Carmel Mountain Ranch. On December 17, 1999, the SANDAG Board approved \$1.4 million in FasTrak funding to operate the revised Inland Breeze (Route 980/990) service plan for two years through December 2001, consistent with the extension of the I-15 pricing project (SANDAG, 1999) .

The goal of funding of Inland Breeze route from Express Lanes revenue was to provide transit riders access to Express Lanes operating during the peak hours. However, most of the transit ridership (75 percent) consisted of those travelling in the reverse commute direction and hence, not using the reversible Express Lanes. Even after the revised transit operations were in place for two years (January 2000- December 2001) the ridership remained low. Therefore, Express lane operations had little impact on transit. In January, 2007 the Inland Breeze route was discontinued primarily because of route duplication, but also somewhat because of low ridership. However, cancellation of the Inland Breeze will not affect current plans to extend the Express Lanes further north and introduce BRT service in 2012 (Chum and Burris, 2008).

\section{Impact on Carpooling}

In a survey conducted in September and October of 1997 (Golob et al., 1999), 95 percent of the ExpressPass users, who used the I-15 corridor before the Express Lanes, mentioned their prior 
mode was driving alone on GPLs. While only 4 percent mentioned they had carpooled prior to Express Lanes. These findings show that the toll paying Express Lanes users came primarily from the SOVs on GPLs and not from carpoolers.

From a later study conducted in 1999 (Golob et al., 2001), a randomly sampled group of "Converted FasTrak Customers" (who converted to FasTrak over the period of study) were found to have the same previous mode choice as the group of "other I-15 Users" (I-15 users except those using the Express Lanes). Table 12 shows the previous modes used by both the groups.

Table 12 Previous Mode for Converted FasTrak Customers and Other I-15 Users

\begin{tabular}{|l|c|c|}
\hline Previous Mode & $\begin{array}{l}\text { Converted FasTrak } \\
\text { Customers }\end{array}$ & $18 \%$ \\
\hline Exclusive Carpool & $14 \%$ & $15 \%$ \\
\hline Both Carpool and Drive Alone & $16 \%$ & $67 \%$ \\
\hline Exclusive Drive Alone & $70 \%$ & \\
\hline
\end{tabular}

These findings indicate that there was not an increased propensity among carpoolers to become FasTrak users. However, the fact that 14 percent of the FasTrak customers exclusively carpooled previously, shows that there is some negative impact on carpooling due to FasTrak.

Comparing the Carpooling trend on I-15 with the control corridor on I-8:The evaluation study team (Wilbur Smith, 2002) selected I-8 as a control corridor. It is an east-west commuter route linking residential areas in the east to downtown San Diego to the southwest. Both freeways, I15 and I-8, are major commuter routes leading toward downtown San Diego. The demographic characteristics of I-8 and I-15 travelers are somewhat comparable. However, the I-8 control corridor does not contain an HOV facility. 
In the monitoring period from 1996 to 1999, traffic in the I-15 corridor experienced a substantial increase in SOV volume and a corresponding decrease in HOV volume during the AM peak period. The majority of this shift occurred between 1997 and 1998 during the transition from the ExpressPass to FasTrak phases of the I-15 pricing project (average daily volume along I-15 increased by 2,672 vehicles but only by 551 persons.). This trend continued during 1998 and 1999.

The decline in HOV volume on the I-15 GPLs contrasted markedly with an observed rise in HOV volume along the I-8 corridor from 1997 to 1999. These results strongly suggested that corridor-specific factors, including the I-15 pricing project, are responsible for these differences (I-8 has no HOV lane). Researchers (Supernak et al., 2002) attributed this decreasing trend of HOV volume along I-15 GPLs to several factors including rising levels of economic prosperity and frustration over lack of access to the Express Lanes, with only one entrance and one exit.

\section{Usage of the Express Lanes}

October 1996 to March 1998 (Phase I Express Pass): During Phase I, total vehicles on the Express Lanes increased by 27 percent from a daily average of 9,215 vehicles in October 1996 (pre-project) to 11,700 vehicles in March 1998. The additional vehicles on the HOV lanes were primarily carpools and not SOVs. HOVs increased from 7,685 daily average vehicles in October 1996 (pre-Express) to 10,790 in March 1998 (a 40 percent increase). By March 1998, ExpressPass users (SOVs) reached 910.

March 1998 to November 1999(Phase II FasTrak): Usage of the I-15 Express Lanes continued to increase during Phase II (see Figure 8). Under the I-15 FasTrak program, average daily traffic on the HOV lanes increased by 29 percent from 11,700 vehicles per day in March 1998 to 15,078 vehicles per day in November 1999.

However, during the same period, SOV usage increased by 287 percent from 910 average daily ExpressPass users in March 1998 to 3,523 average daily FasTrak users in November 1999. FasTrak customers represented approximately 23 percent of total I-15 Express Lanes traffic in November 1999 as compared to 12 percent in March 1998. Average daily carpools using the I- 
15 Express Lanes fluctuated over the same period. As compared to Express Pass Phase, carpools increased at a lower rate under FasTrak. Average, daily carpools increased by six percent from 10,790 in March 1998 to 11,424 in November 1999.

These two findings- increasing carpool usage on the Express Lanes and the decrease in carpooling on the I-15 corridor- seems to be contradictory. However, it should be noted that the former describes the Express Lanes and the later describes the I-15 corridor as a whole. The two opposite trends can be probably due to the fact that the increase in carpools on Express Lanes might have been offset by the decreasing trend on the GPLs, effectively decreasing the total carpool volume on the corridor. Though, it is still unclear how I-8 corridor, without any HOV lanes, managed to have the increasing carpool volume.

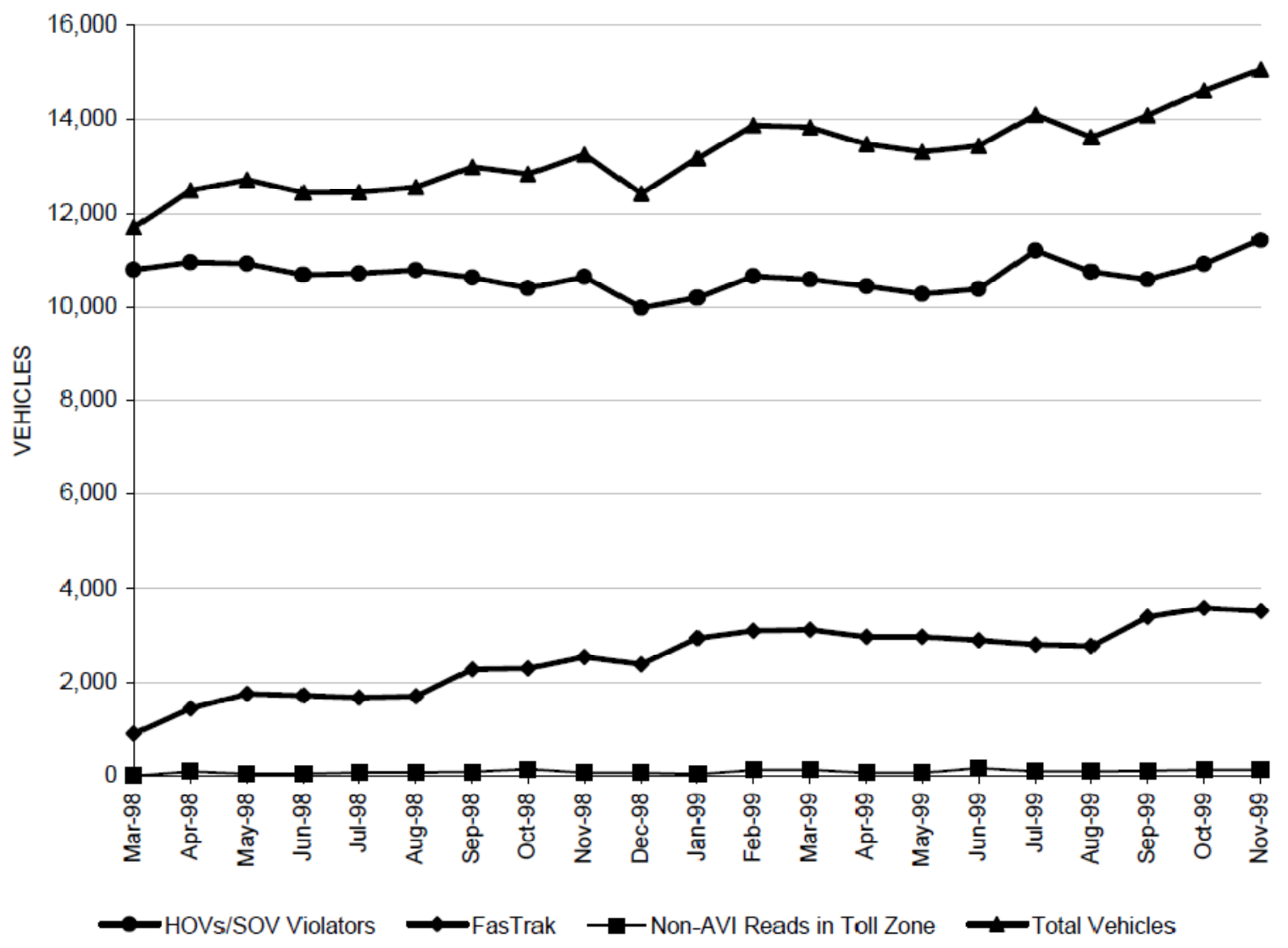

Figure 8 I-15 Express Lanes Daily Average Traffic (SANDAG, 1999) 


\section{Impact on the I-15 Corridor}

During the evaluation period from 1996 to 1999, it was concluded that the Express Lanes did not result in any measurable impact on traffic congestion on the I-15 GPLs. Traffic volumes on the I-15 corridor increased over time (due to Express Lanes as well as due to external factors such as housing growth in the corridor and rerouting of traffic from I-5 (where HOV lane construction caused significant traffic delays). Therefore, even though most of the Express lane users were SOVs who, without the FasTrak program, would otherwise be using the I-15 GPLs, the traffic increase resulted in a relatively flat volume profiles along the I-15 GPLs significantly different from the increasing volume profiles along I-8 from 1996 to 1999. Therefore, as one of its objectives, I-15 pricing project alleviated congestion on the I-15 GPLs only by redirecting an increasing share of volume onto the I-15 Express Lanes (Supernak et al., 2002; SANDAG, 1999).

Average travel speeds in the GPLs in 2001, during the AM peak period (6AM -9 AM), south of Rancho Bernando varied from $55 \mathrm{mph}$ to $60 \mathrm{mph}$ and by 9:00 AM the southbound speed reached at least $60 \mathrm{mph}$. However, during the PM peak, travel speed varied between $20 \mathrm{mph}$ and $35 \mathrm{mph}$ in the vicinity of Miramar Way and Pomerado Road. From Mira Mesa Boulevard to Poway Road travel speeds increased to between $50 \mathrm{mph}$ and $65 \mathrm{mph}$ before slowing again at SR 56/Ted Williams Parkway (Wilbur Smith, 2002). The above findings show that the congestion in the GPLs still existed during the PM peak.

\section{Safety Implications of the Express Lanes}

Most of the Express lane users felt safer in the Express Lanes than in the GPLs. In a survey conducted in September and October 1997, 83 percent of I-15 users considered the I-15 Express Lanes to be safer than the I-15 GPLs (Wilbur Smith, 2002). This difference in safety was perceived most strongly by Express lane users, including both carpoolers ( 84 percent) and ExpressPass users (90 percent).

\section{Revenue for Transit}

One of the objectives of the pricing project was fiscal self-sufficiency and to generate enough revenue to fund continued FasTrak operating costs as well as increased transit service in the I-15 
corridor. The project funded the Inland Breeze (Route 980/990) service. Although one of the goals of the project, funding transit service with the project revenue is not widely supported by I15 FasTrak users and other I-15 commuters. In a survey conducted during the fall of 1998, five percent of respondents indicated that FasTrak revenue should be used to fund the Inland Breeze express bus or other transit service in the I-15 corridor. One-fourth of respondents ( 25 percent) favored using the revenue to extend the I-15 HOV lanes.

The following section discusses the I-25 Express Lanes in Denver. These two Express Lanes are discussed together because of the many operational and design similarities between the two Express Lanes.

\section{I-25 Express Lanes, Denver: Introduction}

The I-25 Express Lanes began in June, 2006 and extend between downtown Denver and US 36 and (see Figure 9). The Express lanes are 7 miles long with two reversible lanes separated from the GPLs by a barrier. There are multiple access points at each end but no intermediate entrances or exits. SOVs are allowed to use the Express Lanes for a fee while HOV2+ and motorcycles can use the lanes for free. In May 2008, 2000 permits were issued to Hybrids for free access to the lanes (CDOT website). The SOV fee varies by the time of day in an attempt to control the demand for the facility. 


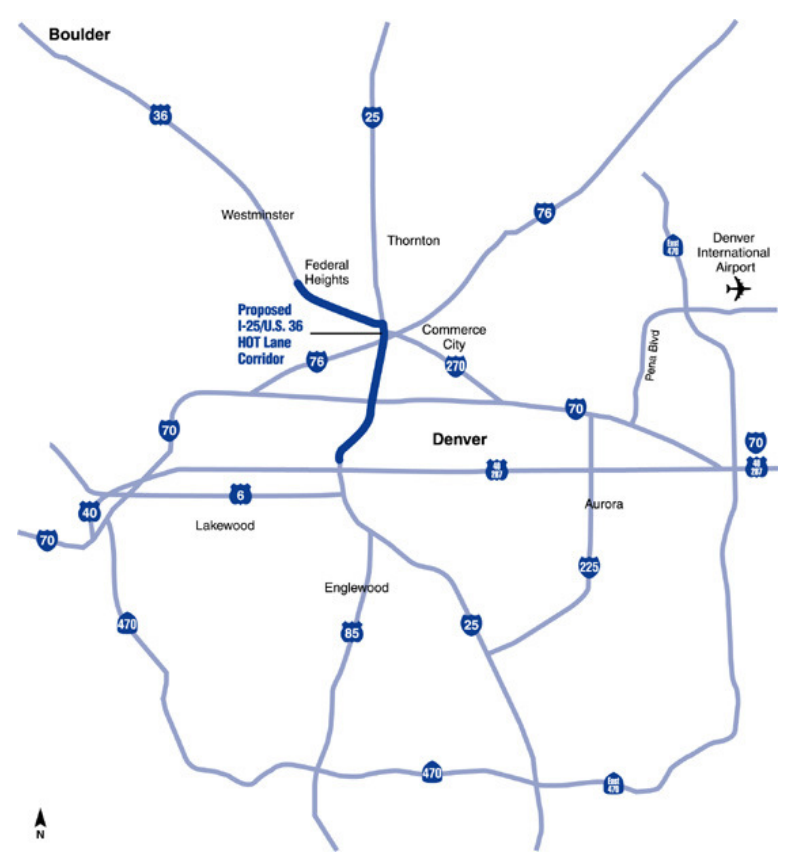

Figure 9 Map Showing Location of I-25 Express Lanes (RITA website)

\section{Why Were Express Lanes Considered?}

In 1999, the Colorado Legislature directed Colorado DOT to convert an HOV lane facility to a Express lane facility that would: “

- use electronic toll collection technology that is compatible with the E-470 toll collection system;

- use variable toll pricing; and

- always operate at level of service "C" or better" (CDOT, 2007).

For the month of June, 1999 in the AM peak period (7AM - 8AM) and PM period (5PM -6 PM) per lane traffic in GPLs averaged a little over 1350 vehicles. Average per lane traffic on the HOV lanes was $600 \mathrm{vph}$ in the PM and $460 \mathrm{vph}$ in the AM (Traffic data obtained from Stacey Stegman, Colorado DOT). The HOV lanes in I-25 were operating far below capacity and growth in their use was slowing. They were able to carry up to 1500 vphpl at level of service C (acceptable system operations). Forecasts prepared by the Denver Regional Council of Governments predicted only 34 percent growth in HOVs between 2001 and 2025, bringing per lane usage to approximately $755 \mathrm{vph}$. These numbers indicated that the HOV lanes would not 
approach their operating capacity by 2025 (Tone, 2009) and there was enough excess capacity to allow toll paying users.

In 2000, CDOT selected the I-25 HOV lane facility as their pilot project candidate. The Colorado Tolling Enterprise (CTE), a division of CDOT, was formed in 2002 to finance, construct, operate, and maintain toll highways in Colorado. On June 2, 2006, the CTE started operations of I-25 HOV/Tolled Express Lanes.

With Express lane operations no changes were made to the access points or the separation mechanism. The only change was the access allowed to SOVs for a toll, and later free access to 2000 Hybrid vehicles.

The Objectives of the I-25 Express Lanes were to (Ungemah et al., 2005):

- Optimize the use of HOV lanes during congested times,

- Provide a new transportation option for North I-25 travelers, and

- Produce enough revenue to cover all expenses, including current operational expenses for the HOV lanes.

There was also a hierarchy of Express lane users, defined as (Tone, 2009):

- Highest priority: Buses and other transit operations

- Second: Vanpools and 3+ person carpools

- Third: Two-person carpools

- Fourth: Inherently low-emitting vehicles (36 mpg [12.6 kpl] or more)

- Lowest priority: Single-occupant vehicles (toll-payers).

The hierarchy of users became an important element in ensuring the Regional Transportation District (RTD) (the area transit provider) and the Denver Regional Council of Governments (DRCOG) [the metropolitan planning organization (MPO)] that Express Lanes were not designed to subvert the use of HOV lanes as a regional mobility and air quality benefit. This hierarchy was further conveyed to the public as a means of addressing the concerns of current users of the HOV facility. 
"The primary purpose of the Express Lanes is not to generate revenue, however, but to:

- Cover operations and maintenance expenses

- Better use the built facility by giving motorists another option to avoid congestion" (Tone, 2009).

\section{How Policies Were Designed Based on Objectives}

Following policies of I-25 Express Lanes have been summarized from the Intergovernmental Agreement (IGA) between Colorado Department of Transportation (CDOT) and the Regional Transport District (CDOT, 2006).

\section{Setting the Minimum Toll}

The public outreach and assessment process for the I-25 Express Lanes identified a strong desire on the part of key stakeholders and transit-advocates that the I-25 Express Lanes project should not give a tacit disincentive to ride the bus by allowing the express bus fare to exceed the prevailing toll. As a result, a minimum toll equal to the RTD Express Bus fare was proposed (currently \$2.75). Note that the minimum toll was proposed to be set regardless of the time of day. However, the minimum toll was restricted only to certain peak hours because of the following complications identified during the planning process-

1) The value decision for the seven-mile HOV facility is not equal to the value decision for the entire length of an express bus ride- an individual's decision to pay a toll versus ride the bus is comprised of not only the travel time benefit of the Express lane, but also the cost savings from not paying gasoline and parking charges by riding the bus. As a result, there is not a true one-toone comparison between riding the bus and paying a toll, from a willingness to pay perspective.

2) a minimum fare only will work when there is sufficient congestion in the general purpose lanes to warrant the minimum fare- the express bus fare is determined by the distance and type of service of the bus - it is not a factor of time of day. The Express Lanes, though, are dependent upon time of day in order to assess the correct toll to manage demand. For example, the Route B from Broomfield to Denver will have the same $\$ 2.75$ fare whether it is used at 8:00 am or 11:00 am. For the Express Lanes, during congested times, the toll rate will be determined by the demand for the facility, and is likely to exceed the minimum toll rate. At uncongested times; the 
GPLs provide a "free" unencumbered trip and of the minimum toll is enforced around that time it is likely very few users would elect to pay the toll due to the availability of uncongested, free GPLs.

Table 13 shows the final toll schedule for the Express Lanes as implemented from the opening day.

Table 13 Toll Schedule for I-25 Express Lanes

\begin{tabular}{|c|c|c|c|}
\hline \multicolumn{2}{|c|}{ AM } & \multicolumn{2}{c|}{ PM } \\
\hline $5: 00-6: 00$ & $\$ 0.50$ & Noon - 3:00 & $\$ 0.50$ \\
\hline $6: 00-6: 45$ & $\$ 1.75$ & $3: 00-3: 30$ & $\$ 1.50$ \\
\hline $6: 45-7: 15$ & $\$ 2.75$ & $3: 30-4: 30$ & $\$ 2.00$ \\
\hline $7: 15-8: 15$ & $\$ 3.25$ & $4: 30-6: 00$ & $\$ 3.25$ \\
\hline $8: 15-8: 45$ & $\$ 2.75$ & $6: 00-7: 00$ & $\$ 1.50$ \\
\hline $8: 45-10: 00$ & $\$ 1.25$ & $7: 00-3: 00$ am & $\$ 0.50$ \\
\hline
\end{tabular}

On January 1, 2009, the morning and afternoon peak hour toll was increased from $\$ 3.25$ to $\$ 3.50$. The toll was increased to comply with the CTE's agreement with Regional Transportation District (RTD) to comply with the bus fare increase in the peak hour. (I-25 Express website)

\section{Toll Triggers}

I-25 Express Lanes are the only Express Lanes for which toll rate triggers are dependent on the travel time of buses. The IGA prescribes following triggers to determine if there is any degradation to travel times for buses and carpool:

- Exceeding target travel time (8 minutes) for more than one bus in an hour (provided the excess is not attributable to a stall, crash and closure of the lane, or special event).

- Exceeding the target travel time for more than one day per week for three weeks in a row.

- Predictable patterns. 
According to the IGA "The travel speed of buses will be the primary means of measuring facility performance. However, total traffic volumes will also be monitored. Even without measured impacts to speeds, growing traffic volumes can indicate a trend that could soon lead to diminished travel speeds. This would also be a trigger to raise toll rates" (CDOT, 2006).

\section{Impact on Transit}

There are two primary transit routes, Route B and Route 120X, and approximately 10 secondary transit routes that use the I-25 Express Lanes.

Table 14 shows the total of ridership of all the routes from 2005 (pre-Express Lanes) through 2009 for the months September and October along with the average gas prices (EIA website) over the same two months. The data was sent by Jeff Dunning, Regional Transport District. It can be observed that there is a general trend of transit ridership increasing and decreasing with gas prices except the year 2006 (Express Lanes started) when gas prices decreased (by 9.5 percent) while the transit ridership remained almost the same.

Table 14 Average Weekday Ridership for Selected Denver Transit Routes

(Regional Transportation District, unpublished data)

\begin{tabular}{|c|c|c|c|c|}
\hline Year & $\begin{array}{c}\text { Gas } \\
\text { Prices }\end{array}$ & Increase (\%) & Transit Ridership & Increase (\%) \\
\hline $\begin{array}{c}\text { September- October } \\
\mathbf{2 0 0 5}\end{array}$ & 283.0 & & 13982 & \\
\hline $\begin{array}{c}\text { September- October } \\
\mathbf{2 0 0 6}\end{array}$ & 256.2 & $-9.5 \%$ & 14034 & $0.4 \%$ \\
\hline $\begin{array}{c}\text { September- October } \\
\mathbf{2 0 0 7}\end{array}$ & 273.1 & $6.6 \%$ & 15148 & $7.9 \%$ \\
\hline $\begin{array}{c}\text { September- October } \\
\mathbf{2 0 0 8}\end{array}$ & 374.2 & $37.0 \%$ & 16926 & $11.7 \%$ \\
\hline $\begin{array}{c}\text { September- October } \\
\mathbf{2 0 0 9}\end{array}$ & 235.0 & $-37.2 \%$ & 14633 & $-13.5 \%$ \\
\hline
\end{tabular}

In terms of transit operations after the Express Lanes opened, the travel time of transit was maintained at the pre-Express Lanes level. Travel time reliability, as measured by transit vehicles, was maintained at an exceptional level (greater than $99 \%$ on-time) for the first four months of Express lane operations in 2006. For October and November 2006, the travel time reliability dipped to a low that still exceeded 90 percent on-time. Significant snow events, which 
dramatically impact the capacity of the facility, were the likely reason for those dips (CDOT, 2007).

In August and September of 2008, a survey was conducted in which respondents included those who most recently used the Express Lanes on US 36 and/ or I-25 in Denver. The survey data was sent by Stacey Stegman, Colorado DOT.

There were indications from the survey that Express lane implementation encouraged more use of transit to some extent. Approximately 12 percent of the Express lane users also agreed to the statement "Because of the Express Lanes I use public transit more often than I otherwise would."

In the survey, respondents were also asked to specify the number of days they used the following modes during the last 7 days- Telecommuting, Transit, Carpooling and Drive alone. Only 62 respondents ( 7 percent of all 832 survey respondents) mentioned days of using the transit.

These findings indicate that among the total survey respondents there was a very small proportion of people using transit (only 7 percent). However, the Express lane may have encouraged more use of transit among some Express lane users. This finding supports the findings in Table 2 in which transit ridership remained almost the same even with decreasing gas price for the year 2006 when Express Lanes opened. This might have been because of the increased usage of transit after the Express Lanes which offset the decrease in ridership due to reduced gas prices.

\section{Impact on Carpooling}

In the August 2008 survey it was found that 54 percent of the respondents, whose only mode of access to Express Lanes was drive alone, mentioned they drive alone (irrespective of lane) for 4 days or more in a week. Almost all of them (98.3 percent) mentioned they did not carpool at all in the last 7 days. These findings indicate that the toll paying customers are primarily driving alone. 
Also, 17 percent of the carpoolers in the Express Lanes mentioned that they carpool in order to use the Express Lanes. This indicates that Express Lanes have been able to encourage carpooling among some travelers.

\section{Trend of Express Lanes Usage}

Figure 10 shows the average weekday usage of Express Lanes by toll users, HOV users and total users. The graph is compiled from monthly Express lane performance reports from June 2006 through July 2009. After the Express Lanes started in June 2006, there is an increasing trend of toll paying users until February 2008 when it levels off and decreases slightly. There is no particular trend of HOV usage and the total number of HOV users has been relatively constant at around 7000 to 8000 .

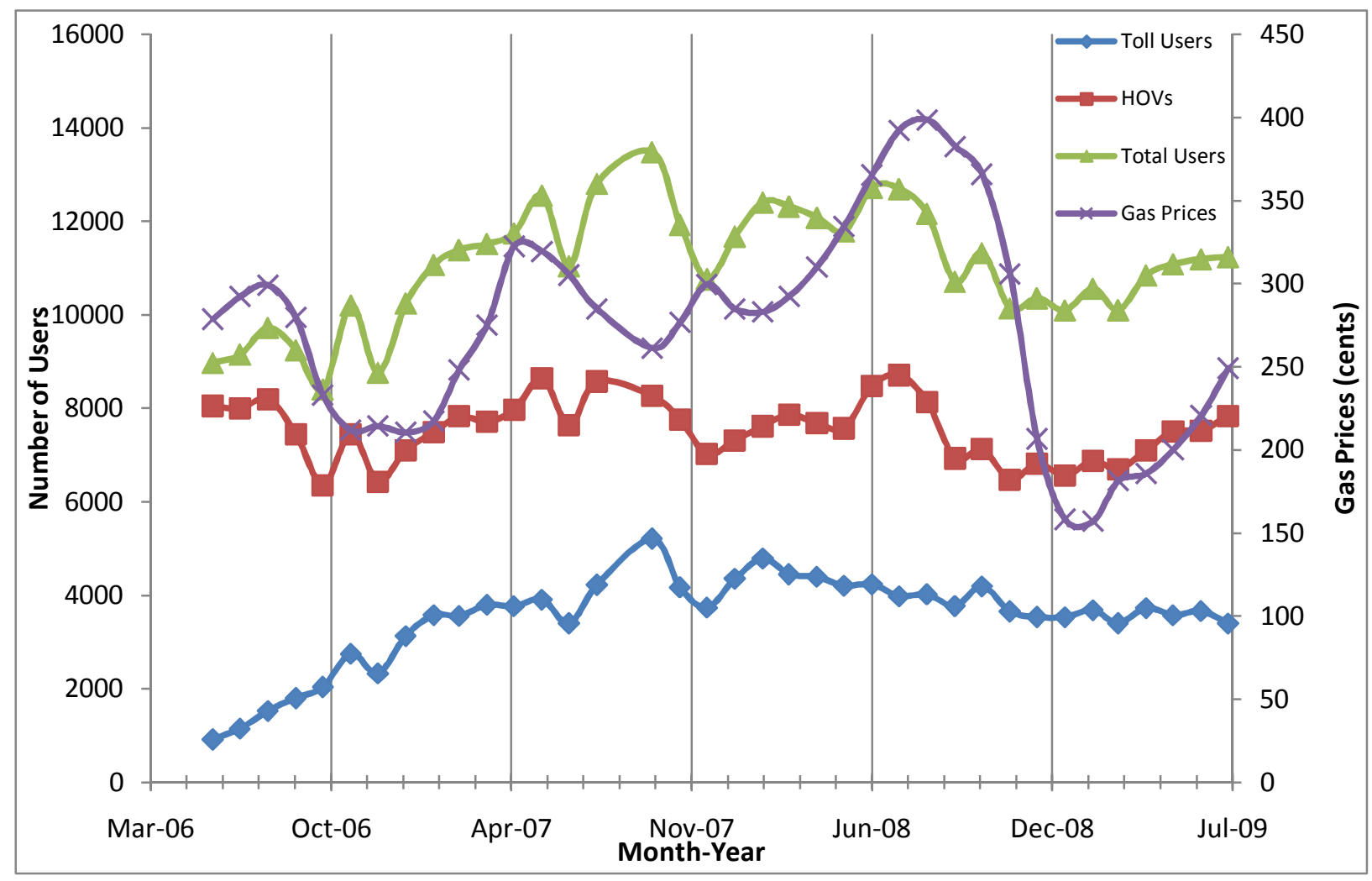

Figure 10 Usage of Express Lanes and Gas Prices (June 2006 Through July 2009) 
To investigate the reasons behind the unusual decrease in Express lane usage by toll paying SOVs (which in all other Express Lanes was increasing in initial years of operation), weekly gas prices (EIA website) in the Denver area were examined.

The month from which the total number of Express lane users started decreasing, July 2008, is the month when the gas prices reached their maximum (\$3.98) (see Figure 10). This decreasing trend is primarily due to the HOV decreasing usage.

HOV usage does appear to be linked to gas price. Toll usage has a generally decreasing trend. It appears that travelers are carpooling more with increasing gas prices. The increasing number of carpoolers is accompanied by a decreasing number of toll paying SOVs potentially indicating carpools being formed from toll users on the Express Lanes. However, no conclusions regarding this mode shift behavior can be made from this data since it would require survey data to confirm any of these mode shifts.

After July 2008, carpool usage as well as toll usage generally decreases (toll usage started decreasing in early 2008). This decrease in Express lane usage can be explained, to some extent, by looking at the increased bus ridership around this period (September and October 2008) in Table 14 which shows that transit ridership increased by almost 12 percent as compared to previous year. However, it is still not clear why the toll usage has a steady decreasing trend irrespective of gas price fluctuations.

In an effort to better understand the decreasing number of toll users on the Express Lanes, the pricing of Express Lanes was examined. The I-25 Express Lanes have a fixed \$3.25 (\$3.50 from January 2009) peak hour toll. There is a possibility that some users would have made an initial decision to use the lanes but over time decided the toll was too high for the time savings. In contrast to this, in a dynamic pricing Express lane, the toll is set based on travel speeds and may be more enticing in situations where all lanes are flowing well (GPL and Express) and therefore the Express Lanes would have a low toll. For example, for the initial six months of operation for I-95 Express Lanes (with dynamic pricing), the average PM peak period toll charged to users was $\$ 1.69$ (\$0.44 for off peak). These arguments call for some research on Express Lanes to 
compare the effectiveness of two pricing mechanisms (variable versus dynamic) in balancing the demand of two user types- tolled and HOVs (and transit) and maintaining the free flow of Express Lanes at the same time.

Another important observation is that the GPLs were not monitored as one of the performance measures. Only Express lane speeds were monitored. This works to optimize the use of HOV lanes and maintain the free-flow travel of transit, but may not lead to optimal pricing.

The other possible reason for decreasing toll usage of the Express Lanes could be the economic recession leading to increased unemployment in the Denver downtown area. Figure 11 shows the unemployment rate in Denver area. After a stable unemployment rate till early 2008, there is a steady increase in unemployment. Interestingly, the decreasing toll usage starts around the same time.

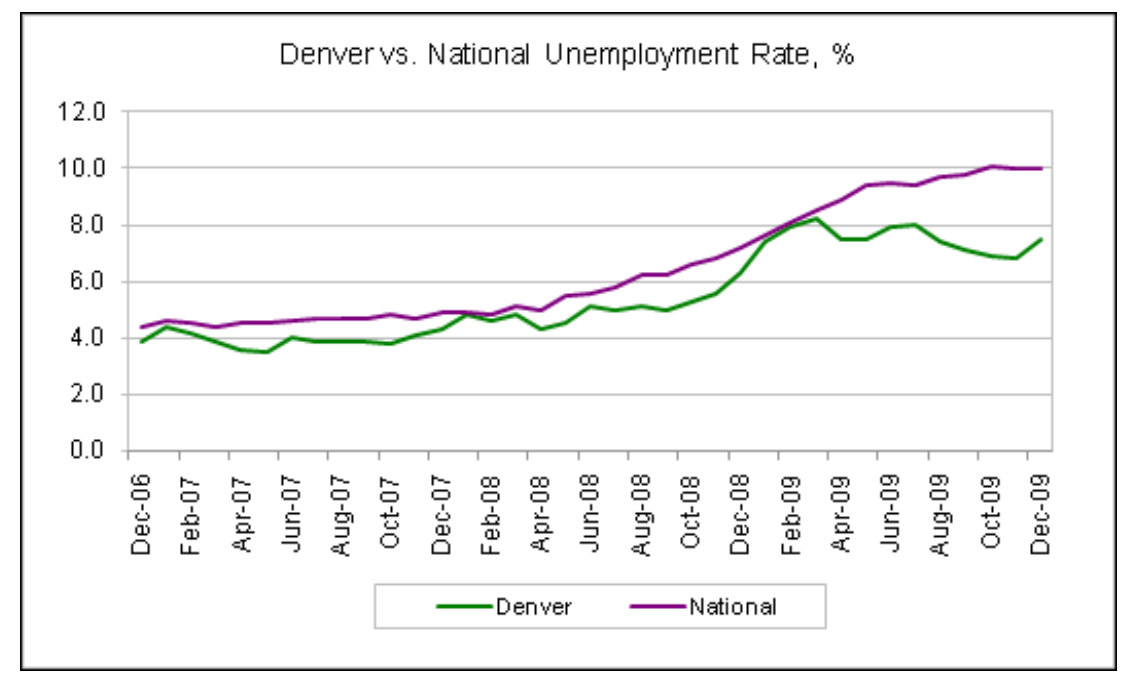

Figure 11 Unemployment Rate for the Denver Region (source: www. Monster.com)

\section{I-15, San Diego Express Lanes and I-25 Express Lanes}

There are many similarities between the I-15 Express Lanes in San Diego (from March 1998 through May 2008) and the I-25 Express Lanes near Denver. Both have two reversible lanes with concrete barrier separation from the GPLs, no intermediate access points and both the Express Lanes have HOV2+ as the carpool eligibility. Both were converted to Express Lanes with no 
design changes to the previous HOV lanes. The lengths are also comparable ( 8 miles and 7 miles respectively) and both the corridors have 4 GPLs in each direction. Therefore, the following section explores these two lanes, their similarities and what can be learned from their differences.

\section{Usage of the Express Lanes}

During the year 1999, when the I-25 Express Lanes were planned, the ADT was around 200,000 vpd while on I-15 the ADT exceeded 250,000 vpd in 1996 when the Express Lanes started. Therefore, the congestion along the two corridors was different.

The I-25 and the I-15 Express Lanes started with similar number of carpools; 7,680 carpools and 8,050 carpools per weekday respectively. Over a period of three years the carpool usage in I-15 Express Lanes increased to 11,400 (November 1999) while it changed very little on I-25.

On the other hand, over the same period, toll users in both the Express Lanes reached almost the same number- 3500 in I-15 and 3400 in I-25. Therefore, the Express Lanes differ in terms of encouraging their carpool usage.

One key difference between the two Express Lanes was that I-15 was implemented because of the underutilized HOV lanes and GPL congestion while I-25 Express Lanes were implemented because of underutilized HOV lane only (as directed by the 1999 legislature). The I-15 corridor was clearly more congested than I-25. This is also reflected in the objectives of the two Express Lanes. I-15 includes the objective of optimizing the use of HOV lanes as well as to reduce congestion on I-15 by providing a toll option to SOVs while I-25 has just the objective of optimizing the use of HOV lanes. Also, I-15 has dynamic pricing while I-25 has fixed variable pricing with a minimum peak hour toll decided by bus fare.

Therefore, it can be argued that the higher congestion level on the I-15 corridor gives travelers more incentive to carpool in the Express Lanes than in I-25 corridor. This may be why I-15 Express Lanes saw an increase of more than 48 percent in the number of HOVs while HOVs on I-25 Express Lanes decreased by 2 percent. 
In terms of supporting services both the Express lanes have park-and-ride lots provided by iCommute program in San Diego and by RTD in Denver. The difference in the available capacities of the park-and-ride lots might influence the difference in the increase in carpooling in the two Express Lanes. However, no conclusions can be made because of lack of information in this aspect.

\section{Safety Issues with the Express Lanes}

Both the Express Lanes have concrete barrier separation and the facilities can be accessed only at the two ends. This provides an opportunity to compare how users perceive the safety of the two Express Lanes.

In a fall 1997 survey of I-15 users (one year after the Express Lanes started), 83 percent considered the I-15 Express Lanes to be safer than the I-15 GPLs. This difference in safety was perceived most strongly by Express Lanes users, including both carpoolers ( 84 percent) and ExpressPass users (90 percent). In a fall 2008 survey of I-25 Express lane users, 89 percent of the respondents were satisfied with the safety aspect of the Express Lanes (for example reduced chances of being in an accident) and 60 percent among them very satisfied. Interestingly, both the Express Lanes have almost equal proportion of respondents satisfied with the safety aspect.

However, the biggest dissatisfaction among I-25 Express lane users came with convenience of access points with almost one-third (32 percent) dissatisfied with this aspect. This dissatisfaction stood out because in almost all other aspects of Express Lanes dissatisfaction was less than five percent. Similarly, in case of I-15, the evaluation team (Supernak et al., 2002) attributed the decreasing carpool volume on I-15 GPLs (from 1996 through 1999) to several factors including rising levels of economic prosperity and frustration over lack of access to the Express Lanes, with only one entrance and one exit. However, no direct perceptions from travelers have been investigated in the I-15 panel surveys.

The above evidence regarding the safety and access aspects of concrete barrier separation indicate that there is a trade-off between perceived safety and access convenience. On one hand, if the concrete barrier between the Express Lanes increases the perceived safety of the Express 
Lanes (perceived my more than 75 percent of the users in both the Express Lanes); it comes with fewer access points (and, more inconvenience to access the Express Lanes). In contrast to this, a concurrent Express lane with many intermediate points will have increased safety issues and the safe operation of Express Lanes depends largely on enforcement efforts and travelers getting used to the access mechanism. 


\section{CHAPTER V}

\section{I-394 EXPRESS LANES, MINNEAPOLIS AND SR 167 HOT LANES,}

\section{SEATTLE}

This chapter examines the two HOT lanes, the I-394 Express lanes in Minneapolis and the SR 167 HOT lanes in Seattle. These were chosen as a pair due to their many similarities.

\section{I-394 HOT Lanes, Minneapolis: Introduction}

The I-394 MnPASS Express Lane project created Minnesota's first Express lanes in May 2005. The HOT lanes run 11 miles between downtown Minneapolis and the western suburbs (see Figure 12 and Figure 13 ). The project allows solo drivers to pay to use the Express Lanes while carpoolers, buses, and motorcyclists may use the lanes free of charge. Tolls are set by dynamic pricing ensuring continued free flow in the lanes at about 50 to $55 \mathrm{mph}$. The Express Lanes consist of two sections. The per-trip fee depends on where users enter and exit the MnPASS Express Lanes. The variable, per-trip fee is always charged for SOV use in the eastern 3 miles from Trunk Highway (TH) 100 to I-94. This section consists of 2 reversible lanes, barrier separated from the GPLs. The western 8 mile of the Express Lanes are separated by paint stripes. This section west of TH 100 is called the diamond lane section and fees are only charged in the peak direction during rush hours (6 AM to $10 \mathrm{AM}$ for the eastbound and $2 \mathrm{PM}$ to $7 \mathrm{PM}$ for the westbound).The lanes are opened to general traffic at other times. The fee is posted on changeable message signs located just before entrances to MnPASS lanes. The fee can be adjusted as often as every three minutes. The tolls range from 25 cents to $\$ 8$ and average $\$ 1$ to \$4 during rush hour (MnPASS website).

\section{Previous HOV Lanes Operations}

Historically, the reversible lane section of HOV lanes was open only to bus and carpools with two or more passengers in the inbound (eastbound) direction (from 6:00 AM to 1:00 PM), and open in the outbound (westbound) direction (from 2:00 PM to midnight) on weekdays. These lanes were also opened to buses and HOV traffic on a limited basis on weekends, usually in support of special event traffic. The lanes were closed at all other times. 
Prior to the introduction of MnPASS, the diamond lane section was designated for use by carpools and transit vehicles during the morning commute period (6:00 AM to 9:00 AM) for the inbound direction, and during the afternoon commute period (3:00 PM to 6:00 PM) for the outbound direction. The HOV restrictions on this section of the corridor were only applied on weekdays and the lane was available for use by all traffic for the remaining hours of the day.

\section{Why Were HOT Lanes Considered?}

Shortly after the HOV lanes opened, congestion in the GPLs in addition to a less-than-full HOV facility created a public perception that the HOV lanes were underutilized. This perception persisted for the decade since I-394 opened and led to periodic requests that the HOV lane be opened to solo drivers throughout the day. This culminated with a request by the Legislature that Mn/DOT conduct a study to evaluate the feasibility of this action. The study was completed in 2001 and concluded that the HOV facility was underutilized but that opening it to general traffic would not be cost effective and would result in a congested facility during the peak periods. The same study concluded that conversion to a High Occupancy Toll (HOT) lane operation would be the most cost-effective action. (MnDOT, 2004)

\section{Objectives of HOT Lanes}

Mn/DOT directed the implementation team to design and deploy a HOT lane system that met five project objectives (Cambridge Systematics, 2006a):

1. Improve the efficiency of I-394 by increasing the number of people and vehicles using the HOV lanes.

2. Maintain free-flow speeds for transit and carpools in the express (HOV) lanes.

3. Use excess revenues, if available, to make transit and highway improvements in the I-394 corridor.

4. Use electronic toll collection (i.e., tags/transponders and readers) which do not require toll booths.

5. Employ new Intelligent Transportation System (ITS) technologies, such as dynamic pricing and in-vehicle electronic enforcement. 


\section{(}
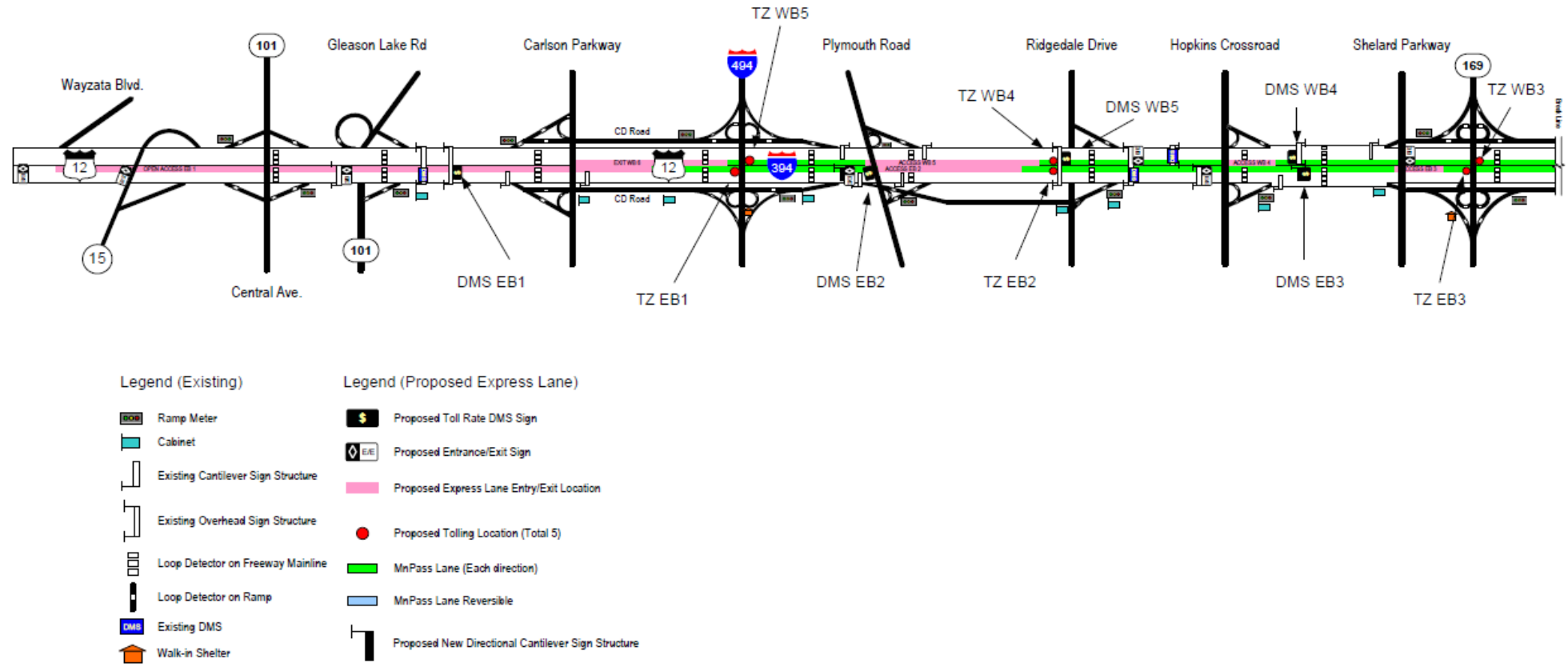

MnPass System Concept MnPASS 12 12/904 Figure 1A

Figure 12 I-394 West Section from Wayzata Boulevard to TH 169 (source: www.mnpass.org) 


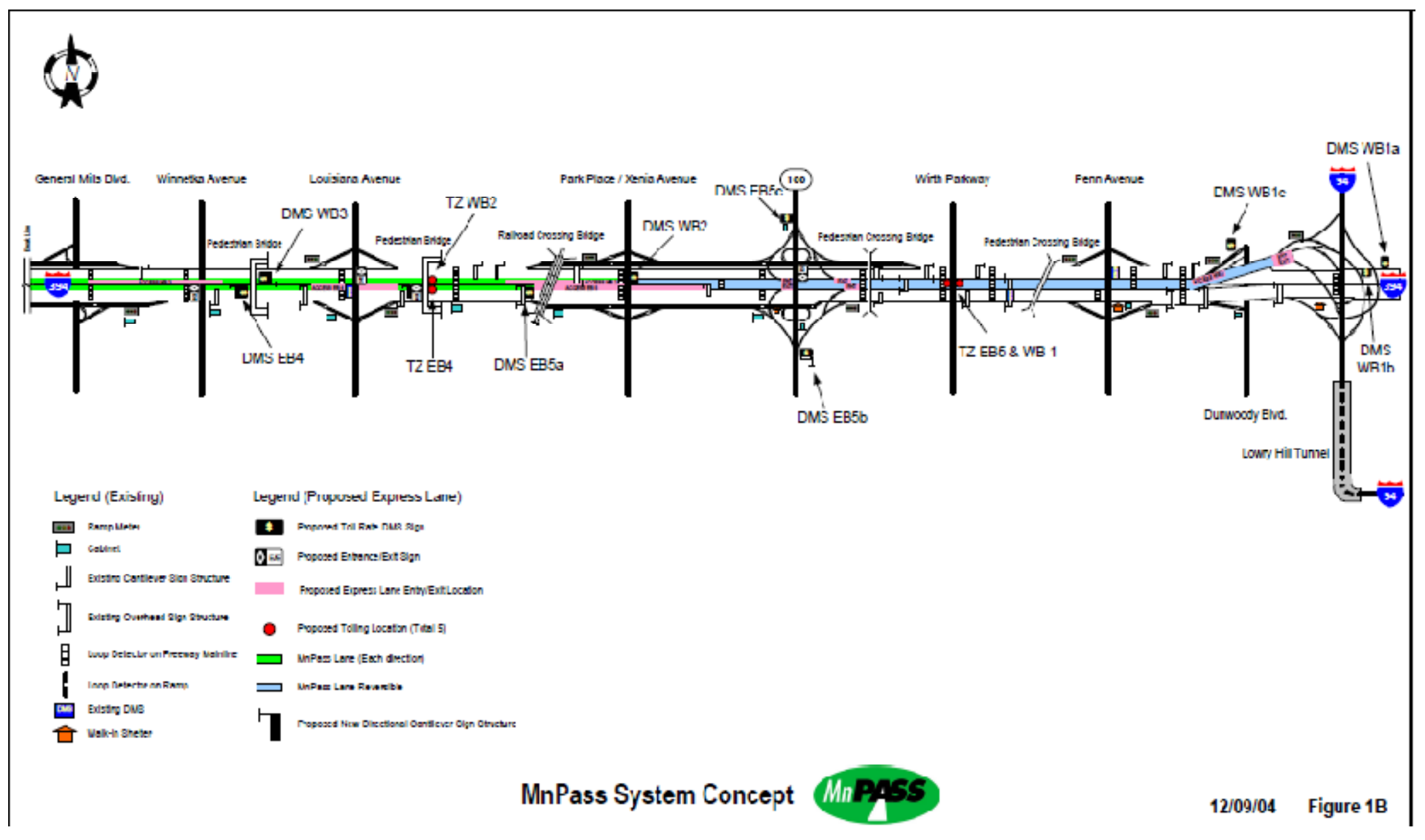

Figure 13 I-394 East Section from General Mills Boulevard (source: www.mnpass.org) 


\section{Important Issues during HOV to HOT Conversion}

Separation Mechanism: The MnPASS project was the first HOT lane to use the solid double white-striped lines rather than barriers to separate the Express Lanes from the GPLs (for the diamond lane section of I-394). Before MnPASS, the two HOT lanes in California (SR 91 and I15) and the two in Texas (I-10 and US 290) were separated by fixed barriers with limited access. However, physical design and space limitations on the I-394 facility constrained the possibility to install barriers to separate the facility from the adjacent GPLs without major construction and cost. Also, a facility with barrier separation would have also greatly limited access to and from the facility, and such access was necessary to facilitate transit and carpooling (Munnich and Buckeye, 2007).

Access points: Before the Express Lanes, carpools, vanpools and buses could access the 8-mile diamond section anywhere, but after the Express Lanes became operational there were designated points for access and egress and it was illegal to cross the double white line separating the Express Lanes from the GPLs. Serious consideration was given to allowing the HOVs and buses to cross the double-white lines and give them unrestricted access to HOT lanes as they had when the lanes were HOV lanes. However, unrestricted access was ruled out because of the following reasons:

- Unrestricted access for buses will lead to merging problems between the slow moving buses (at any location) and the faster moving Express Lanes vehicles.

- Detailed modeling of bus operations indicated that there would be negligible delay to buses due to restricted access.

- Unrestricted access to HOVs would mean verifying occupancy of all the vehicles crossing the double white lines

- SOVs could take advantage of unrestricted access by moving in and out lanes to avoid the tolls (MnDOT, 2004).

There are 11 access points for the I-395 MnPASS project, five in the eastbound and six in the westbound. The access points are approximately 0.25 to 0.75 miles in length. The access points include visual enforcement of occupancy levels and electronic toll tag readers. (Turnbull, 2005) 
Hours of operation for the diamond lane section: The diamond section of the HOV lanes from I494 to TH-100 was limited to HOV traffic during the weekday morning peak commuting period in the eastbound direction (towards downtown -6AM to 9 AM) and on weekday evenings in the westbound direction (3 PM to $6 \mathrm{PM}$ ). During the off-peak period and in the reverse direction, the HOV lanes were available for general use. With the implementation of Express Lanes, the lanes were priced 24x7 for the following reasons (MnDOT, 2004):

- A $24 \times 7$ operation would result in the least confusion among drivers (putting a sign of "open lanes" or "zero tolls" were considered in the off peak periods), and that the alternative would create significant enforcement problems with the new double white-striped lanes (SOVs entering the lanes before the hours of operation and being in the lane when tolling starts).

- The consulting team argued that there should be a minimum toll at all times to ensure an adequate level of revenue, though it was unclear at the time how much of a revenue impact the minimum toll would have.

There was a vigorous debate over this issue among the members of the community task force. The opponents of the $24 \times 7$ approach felt it would be a bad idea to take away the existing access to the HOV lanes during off-peak periods.

Shortly after the I-394 MnPASS lanes opened, commuters in the eastbound (reverse-commute) direction (for which diamond lanes were previously opened to general traffic) between I-94 and Highway 169 began to experience increased congestion in the GPLs leading to a major public uproar, and a quick reversal by MnDOT to peak-only operation after four weeks of operation. When the lanes were reopened to all traffic in the reverse-commute direction, the congestion, negative calls, and media coverage quickly subsided (Munnich and Buckeye, 2007). One difference was that, operational hours were modified to a slightly expanded version of the previous operational hours. The current operational hours for the MnPASS lane in the diamond section are 6:00 AM to 10:00 AM for the inbound direction (an addition of 1 hour of morning commute period HOV restrictions compared with historical hours), and 2:00 PM to 7:00 PM for 
the outbound direction (an addition of 2 hours of afternoon commute period HOV restrictions compared with historical hours).

\section{Impact on Transit}

When planning for an HOV-to-HOT lane conversion (Weinstein and Sciara, 2004) began in Minnesota, the advocacy group "Transit for Livable Communities" advocated for a project that would improve bus service in the corridor. The Minnesota legislature directed that 50 percent of HOT lane revenues in excess of project costs be spent on transit in the corridor. In addition, if a second phase of the project goes forward, a third HOT lane would be created in the barrierseparated portion of the I-394 corridor. A moveable barrier design would provide two lanes in the peak direction and one lane in the reverse commute direction, thus allowing buses (as well as carpools and toll paying SOVs) to use the lane in the reverse commute direction, adding additional benefits to the bus rapid transit service in the corridor.

During July-September 2005, shortly after the MnPASS lanes opened, transit ridership along I-394 increased by more than 13 percent over the ridership from the same period in 2004, before MnPASS was available (see Table 15). In comparison, transit ridership in the I-35W corridor increased only 1.4 percent. Chum and Burris (2008) indicated some possibility of bus ridership being positively affected by the adaptation of HOV to HOT lanes. However, there was no specific study to determine the effect that having a new SOV toll option had on existing transit users and it was unknown how many former transit users switched to the SOV toll mode.

Both Turnbull (2008) and John et al. (2006) observed that the transit usage remained relatively constant after the MnPASS implementation and it was due to the growth limited by the park and ride lots which were mostly at capacity. 
Table 15 Average Peak Period Transit Ridership for Third Quarter (3Q) on Minneapolis HOV and HOT Lanes (Mn/DOT, unpublished data).

\begin{tabular}{|c|c|c|c|c|c|}
\hline & \multicolumn{3}{|c|}{ Average Peak Period Ridership } & \multirow{2}{*}{\begin{tabular}{|l|}
$2004-05$ \\
Change
\end{tabular}} & \multirow{2}{*}{$\begin{array}{l}2005-06 \\
\text { Change }\end{array}$} \\
\hline & 3Q 2004 & 3Q 2005 & 3Q 2006 & & \\
\hline \multicolumn{6}{|l|}{ I-394 } \\
\hline EB Reversible & 3594 & 4014 & 4293 & $11.7 \%$ & $7.0 \%$ \\
\hline WB Reversible & 3138 & 3569 & 3673 & $13.7 \%$ & $2.9 \%$ \\
\hline Total Reversible & 6687 & 7583 & 7966 & $13.4 \%$ & $5.1 \%$ \\
\hline EB Diamond & 2312 & 2635 & 2970 & $14.0 \%$ & $12.7 \%$ \\
\hline WB Diamond & 2026 & 2323 & 2510 & $14.7 \%$ & $8.0 \%$ \\
\hline Total Demand & 4338 & 4958 & 5480 & $14.3 \%$ & $10.5 \%$ \\
\hline \multicolumn{6}{|l|}{ I-35 W } \\
\hline NB & 1251 & 1300 & 1351 & $3.9 \%$ & $3.9 \%$ \\
\hline SB & 1109 & 1092 & 1136 & $-1.5 \%$ & $4.0 \%$ \\
\hline Total & 2360 & 2392 & 2487 & $1.4 \%$ & $4.0 \%$ \\
\hline
\end{tabular}

Bus Ridership and Gas Prices: As observed by the evaluation team of MnPASS, "gasoline prices reached all time high in the region during the evaluation period, increasing from approximately $\$ 1.95$ a gallon at the time of MnPASS opening (May 2005) to over \$3.00 a gallon by July 2006." (Cambridge Systematics, 2006a). To further investigate any potential relationship between the transit ridership and gas prices, the weekly gas price for the third quarters of the years 2004 through 2006 were obtained (EIA website). It was observed the increase in gas prices from 2004 to 2005 (\$1.80 to $\$ 2.42$ ) and from 2005 to 2006 (\$2.42 to $\$ 2.75$ ) is followed by increase in ridership during those years by 14 percent and 10 percent respectively. These findings indicate that gas price might have impacted the transit ridership.

Bus Ridership: Next, the relatively large increase in transit ridership on the I-394 corridor as compared to the I-35 corridor was investigated. In terms of bus operations there were no changes made due to Express Lanes (except the change in their access mechanism which was changed 
from unrestricted along the length of HOV lanes to some designated points on Express Lanes). The travel speeds increased by a maximum of $3 \mathrm{mph}$ on Express Lanes.

However, there are indications from the panel surveys of transit users that their propensity to use transit did not decrease over time. For the panel surveys conducted in fall 2005 and spring 2006 (after the Express Lanes were implemented), a panel of transit users was asked "Now consider all trips you made in both directions. On how many of those trips did you use the following modes? - SOV on HOT, SOV on GPLs, Carpool, Vanpool or Transit (Zmud, 2006). In 2005, respondents mentioned they made 48 percent of the trips using the transit and only one percent trips as SOVs on Express Lanes. The panel survey conducted in the Spring of 2006(Zmud, 2006a), one year after the Express Lanes became operational, when transit users were asked the same question, transit trips made by transit users increased to 54 percent and the HOT lane trips remained one percent. These findings indicate that the transit users make almost no trips by Express Lanes as SOVs and the propensity to use the transit did not decrease with Express lanes.

Bus Operations: To evaluate any impacts on transit operational performance associated with the I-394 MnPASS Express Lanes deployment, supervisors of the four local and regional transit providers were interviewed in 2006 (Cambridge Systematics, 2006a). Among them, three agencies indicated that the deployment of MnPASS on I-394 generally had a negligible impact on their operations in the I-394 corridor. These providers were also unable to identify any positive impacts directly attributable to MnPASS. While there was support voiced for the added hours of HOV restrictions on the diamond lane section, the agencies did not report any quantifiable impacts resulting from expansion of operational hours.

Southwest Metro Transit was the only agency that voiced serious concerns regarding operational impacts related to the deployment of MnPASS on I-394. Specifically, the agency cited added difficulty in merging from northbound TH 169 to eastbound I-394, reporting that the limited merge area, combined with added traffic in MnPASS lane and aggressive behavior by drivers in the MnPASS lane resulted in difficulty for transit vehicle drivers in finding appropriate gaps in which to merge the vehicle into the MnPASS lane at this location. Transit vehicle drivers have reported that drivers in the MnPASS lane will often attempt to close any gaps ahead of their 
vehicles when they see a transit vehicle attempting to merge ahead of them, making it more difficult for the transit vehicle to successfully merge into the lane (Cambridge Systematics, 2006a).

Other transit providers did not report similar merge issues; however, Southwest Metro Transit does operate a greater number of buses through the TH 169 to I-394 interchange than the other providers and may have more opportunity to experience these particular impacts.

\section{Impacts on Carpooling}

Comparing the mode used by panel survey respondents in the Fall of 2004 (pre- MnPASS) to Spring 2006, I-394 panelists were less likely to switch from SOV to carpool than were those in the I-35W control corridor (7 percent versus 10 percent). Those in the control corridor were more likely to switch from carpool to SOV (8 percent versus 20 percent). Therefore, Express Lanes did not have a negative impact on carpooling.

In contradiction to the panel surveys' results showing few of I-394 carpoolers shifting to SOVs, the vehicle occupancy counts after the Express Lanes during the peak periods 6 AM to 9 AM and $3 \mathrm{PM}$ to $6 \mathrm{PM}$ show that the carpooling decreased on HOV lanes as well as GPLs. Comparing three hour peak periods for HOV lanes in 2004 by 2006- on the reversible section carpooling decreased by 21 percent in AM peak and by 25 percent in the PM peak. On diamond section carpooling decreased by 26 percent in the AM peak and by 45 percent in PM peak. The decrease in the carpooling in I-394 was more substantial than in I-35W control corridor. The technical evaluation team attributed this to the shift of some HOVs to the off peak hours (which were extended for diamond lane section- 2 hours in AM and 1 hour in PM) however, the reasons for the decreased carpooling were inconclusive (Cambridge Systematics, 2006a).

\section{Usage and Tolls}

The price of the toll (ranging from $\$ 0.25$ to $\$ 8.00$ ) varies with the current congestion levels and with the distance traveled. A different toll is paid whether the MnPASS subscriber chooses to travel on the reversible section, the diamond lane section, or both. A modified toll rate structure was implemented in January 2006. With the modified structure (Cambridge Systematics, 2006a) 
although the range of possible tolls remained unchanged, but higher average tolls were applied as increases in toll levels were triggered by lower congestion levels. Immediately following this change, the number of weekly tolled trips immediately decreased by 3,000 to 4,000(see Table 16). However, since then, the number of tolled trips steadily increased and, reached at a level higher than usage before the rate change in six months.

Table 16 Comparison of Usage and Revenue Before and After Rate Change

\begin{tabular}{|l|c|c|c|}
\hline & $\begin{array}{c}\text { September 2005 } \\
\text { (Before rate change) }\end{array}$ & $\begin{array}{c}\text { September 2006 } \\
\text { (After rate change) }\end{array}$ & \% Change \\
\hline Average Toll & $\$ 0.55$ & $\$ 1.10$ & $100 \%$ \\
\hline Average Daily Trips & 3500 & 4000 & $14 \%$ \\
\hline
\end{tabular}

Rate changed in January 2006

\section{Vehicle and Person Throughput}

Table 17 shows the performance of Express Lanes before (2004) and after (2007) the Express Lanes became operational. The 2004 data was obtained from MnPASS technical evaluation report (Cambridge Systematics, 2006a) and 2007 data was obtained from the quarterly reports (MnDOT, 2008) issued by MnPASS. The table shows the percentage of people and vehicles moved on HOV/Express Lanes out of the total facility (HOV/HOT and GPLs). The table also shows the percentage capacity of HOV/Express Lanes out of the total capacity of the facility. For the 2004 data, number of buses out of total HOVs was unknown which was assumed to be equal to that in 2007 (116 in buses, which is safe assumption) and the vehicle occupancies were taken as 2.1 for HOVs, 1.15 for SOVs, 27.26 as mentioned in quarterly reports. (Note that the aim of the table is to show an approximate comparison between the person and vehicle throughput of the HOV lanes before and after conversion). 
Table 17 Comparison of Person Throughput and Vehicle Throughput Before and After the HOT Lanes

\begin{tabular}{|c|c|c|c|c|c|}
\hline & \multicolumn{2}{|c|}{$\begin{array}{c}\text { Before HOT lanes } \\
(2004)\end{array}$} & \multicolumn{2}{|c|}{$\begin{array}{c}\text { After HOT lanes } \\
(2007)\end{array}$} & \\
\hline \multicolumn{6}{|c|}{ Reversible Section } \\
\hline & $\begin{array}{l}\text { \% People } \\
\text { moved }\end{array}$ & $\begin{array}{l}\% \\
\text { Vehicles }\end{array}$ & $\begin{array}{l}\text { \% People } \\
\text { moved }\end{array}$ & $\begin{array}{l}\% \\
\text { Vehicles }\end{array}$ & $\begin{array}{l}\text { \%Capacity of HOV/HOT } \\
\text { Lanes }\end{array}$ \\
\hline $\begin{array}{l}\text { AM } \\
\text { Peak }\end{array}$ & 40 & 18 & 43 & 22 & $\begin{array}{l}40 \%(3 \text { GPLs and } 1 \text { aux* lane, } 2 \\
\text { HOV lanes) }\end{array}$ \\
\hline $\begin{array}{l}\text { PM } \\
\text { Peak }\end{array}$ & 30 & 21 & 39 & 19 & 40\%(3 GPLs, 2 HOT lanes) \\
\hline \multicolumn{6}{|c|}{ Diamond Section } \\
\hline & $\begin{array}{l}\text { \% People } \\
\text { moved }\end{array}$ & $\begin{array}{l}\% \\
\text { Vehicles }\end{array}$ & $\begin{array}{l}\text { \% People } \\
\text { moved }\end{array}$ & $\begin{array}{l}\% \\
\text { Vehicles }\end{array}$ & $\begin{array}{l}\text { \%Capacity of HOV/HOT } \\
\text { Lanes }\end{array}$ \\
\hline $\begin{array}{l}\text { AM } \\
\text { Peak }\end{array}$ & 35 & 16 & 39 & 19 & $33 \%(2$ GPLs, 1 HOV lane) \\
\hline $\begin{array}{l}\text { PM } \\
\text { Peak }\end{array}$ & 46 & 27 & 25 & 11 & $\begin{array}{l}33 \%(2 \text { GPLs and } 1 \text { aux** lane, } \\
1 \text { HOV lane) }\end{array}$ \\
\hline
\end{tabular}

*Auxiliary lanes not included in capacity

**Auxiliary lane in this direction was added after the Express Lanes in 2005

From the above table, for reversible section, it can be observed that the person throughput in AM and PM peaks increased after the Express Lanes. Also, in PM peak the person throughput increased with a decrease in the percentage of vehicles.

For diamond lane section, in the AM peak the person throughput increased with increase in vehicle throughput and in the PM peak, the vehicle throughput reduced to half with a reduction of more than half of the vehicles. This is possible because of additional 2 hours of HOT lane operations in PM peak.

The above findings indicate that in almost all the cases, the person throughput is not negatively impacted due to HOT lane operations. As one of the objectives of I-394 Express Lanes is to increase the number of people using the HOV lanes, the above findings indicate that Express Lanes are achieving this objective.

Also, looking at the peak hour traffic of Express Lanes in 2008 (MnDOT, 2008), the Express Lanes still have enough of their capacity available to maintain the free flow (assuming $1500 \mathrm{vph}$ to be the capacity of each lane for maintaining the free flow). 


\section{Speed of the HOT Lanes and the GPLS}

Table 18 and Table 19 show the speed of HOV lanes and GPLs before (July 2003- May 2005) and after (May 2005- July 2006) the Express Lanes started. In this table all the locations except Penn (Pennsylvania Avenue) are the reversible section of the I-394 HOT lanes (see Figure 13). There was a small increase in speed in both the Express Lanes as well as GPLs after the Express Lanes and this lowered the speed differential between the GPL and HOT lane to some extent (from a maximum of $13.4 \mathrm{mph}$ to $9.7 \mathrm{mph}$ ). The findings indicate that the addition of toll paying SOVs on the Express Lanes did not cause a reduction on the speed of Express Lanes and the speed of GPLs increased. Therefore, the MNPASS Express Lanes successfully achieved their objective of maintain free flow speed for transit and carpools in the HOV lanes.

Table 18 Comparison of Pre- and Post- MnPASS speeds in the MnPASS Lanes (Cambridge Systematics, 2006a)

\begin{tabular}{|l|c|c|c|}
\hline Location/Direction & Pre-MnPASS & With MnPASS & Difference \\
\hline Eastbound (AM Peak Period) & 71.2 & $0.1 \%$ \\
\hline I-494 & 71.1 & 69.4 & $4.0 \%$ \\
\hline Ridgedale & 66.7 & 65.2 & $3.3 \%$ \\
\hline General Mills & 63.1 & 60.8 & $-2.4 \%$ \\
\hline Xenia & 62.3 & 67.1 & $0.4 \%$ \\
\hline Penn & 66.8 & \multicolumn{2}{|l|}{} \\
\hline Westbound (PM Peak Period) & 66.7 & 67.9 & $1.8 \%$ \\
\hline Penn & 57.2 & 57.2 & $0.0 \%$ \\
\hline Xenia & 62.3 & 64.3 & $3.2 \%$ \\
\hline General Mills & 66.3 & 68.6 & $3.5 \%$ \\
\hline Ridgedale
\end{tabular}


Table 19 Comparison of Pre- and Post- MnPASS Average Speeds on the GPLs (Cambridge Systematics, 2006a)

\begin{tabular}{|l|c|c|c|}
\hline Location/Direction & Pre-MnPASS & With MnPASS & Difference \\
\hline Eastbound (AM Peak Period) \\
\hline I-494 & 67.2 & 68.1 & $1.3 \%$ \\
\hline Ridgedale & 63.8 & 66.5 & $4.2 \%$ \\
\hline General Mills & 57.5 & 65.9 & $14.6 \%$ \\
\hline Xenia & 51 & 57.1 & $12.0 \%$ \\
\hline Penn & 55.1 & 57.1 & $3.6 \%$ \\
\hline Westbound (PM Peak Period) & 59.6 & $10.8 \%$ \\
\hline Penn & 53.8 & 47.6 & $8.7 \%$ \\
\hline Xenia & 43.8 & 72.2 & $1.8 \%$ \\
\hline General Mills & 70.9 & 63.1 & $4.1 \%$ \\
\hline Ridgedale & 60.6 & 65 & $0.5 \%$ \\
\hline I-494 & 64.7 & \multicolumn{2}{|l}{} \\
\hline
\end{tabular}

\section{Safety Implications of the Express Lanes}

Since, there was no previous experience of operating a HOT lane only with double white line separations from the GPLs, authorities were concerned regarding the enforcement of the separation mechanism, excessive weaving between lanes at the entry and exit points, space for drivers to get into or out of the MnPASS lanes, and if the separation mechanism would result in more accidents (Munnich and Buckeye, 2007).

In a panel survey conducted six months after the Express Lanes started; the safety issue did not surface among the survey responses. Among the MnPASS users, more than three-quarters of the respondents were satisfied with the ease of identifying the MnPASS entry points, with 39 percent very satisfied. Most respondents (66 percent) were satisfied with the safety of merging into the MnPASS lanes, with one-fourth very satisfied. Respondents who used the MnPASS lanes were asked if they experienced any problem in merging in to the MnPASS lanes from the GPLs- 87 percent of the respondents said "No" and only 13 percent said "Yes" (MnDOT, 2004).

One bus company representative remarked that the double-striped lines are actually safer for buses than dashed lines, since drivers can only pull in front of buses at designated entry and exit points (Munnich and Buckeye, 2007). The MnPASS project also included funding the enforcement activities for the Express Lanes. 
Between May and December 2005 (the first 8 months of HOT lane operations), public safety officers made nearly 5,000 stops on the I-394 corridor. Approximately 50 percent of these stops involved HOV violations or motorists illegally crossing the double white lines. This increased enforcement effort appeared to have a positive impact on compliance as HOV violation rates decreased compared with conditions in the years prior to MnPASS, and the violation rates in the I-394 corridor were significantly lower than on HOV facilities on I-35W (Cambridge Systematics, 2006a).

Accidents on I-394 have declined 12 percent, from an annual average of 560 during the 2 years preceding the opening of the MnPASS lanes to 493 for the year immediately following the opening. Entering and exiting the MnPASS lanes has not been a significant problem, and drivers have generally respected the double-white lines (Munnich and Buckeye, 2007).

Though safety was not one of the five specific objectives of I-394 Express Lanes it is always a critical issue for any project. This is particularly true with MnPASS being the first HOT to use a double white line separation mechanism and the project planners were skeptical about the safety implications of this separation mechanism. This separation mechanism was since there was a lack of space on the freeway to provide barrier separation. Therefore, to know the impact of such separation mechanism on HOT lane operations is important for I-394 as well as future HOT lanes which might consider this as their separation mechanism. For example SR 167 HOT lanes in Seattle based their separation mechanism on the I-394 double white line separation mechanism.

\section{Revenue for the Transit}

As one of the objectives, half of the MnPASS excess revenue was required to be directed to the transit and this was critical in gathering the support for the project. The MnPASS project is generating sufficient revenue to cover operating costs, but the project has not generated any excess revenue (after covering the capital cost) to support the transit (Munnich, 2010). 


\section{SR 167 HOT Lanes, Seattle: Introduction}

The SR 167 HOT lanes are located near Seattle. The HOT lanes began operation in May of 2008, with a single HOT lane running in each direction of SR 167 for approximately nine miles between Renton and Auburn (see Figure 14). SOVs are allowed to use the lanes by paying a toll using a transponder. Tolls for the HOT lanes are set to ensure that vehicles using these lanes travel at 45-60 mph even when the GPLs are congested. Toll rates vary depending on the level of congestion to ensure that traffic in the HOT lane always flows freely and carpools enjoy the same fast and reliable trip they had when they allowed only HOVs. Two-person carpools, vanpools, transit and motorcycles can use the lanes for free. The highway's two general purpose lanes in each direction remain toll free and open to all (SR 167 HOT lanes website).

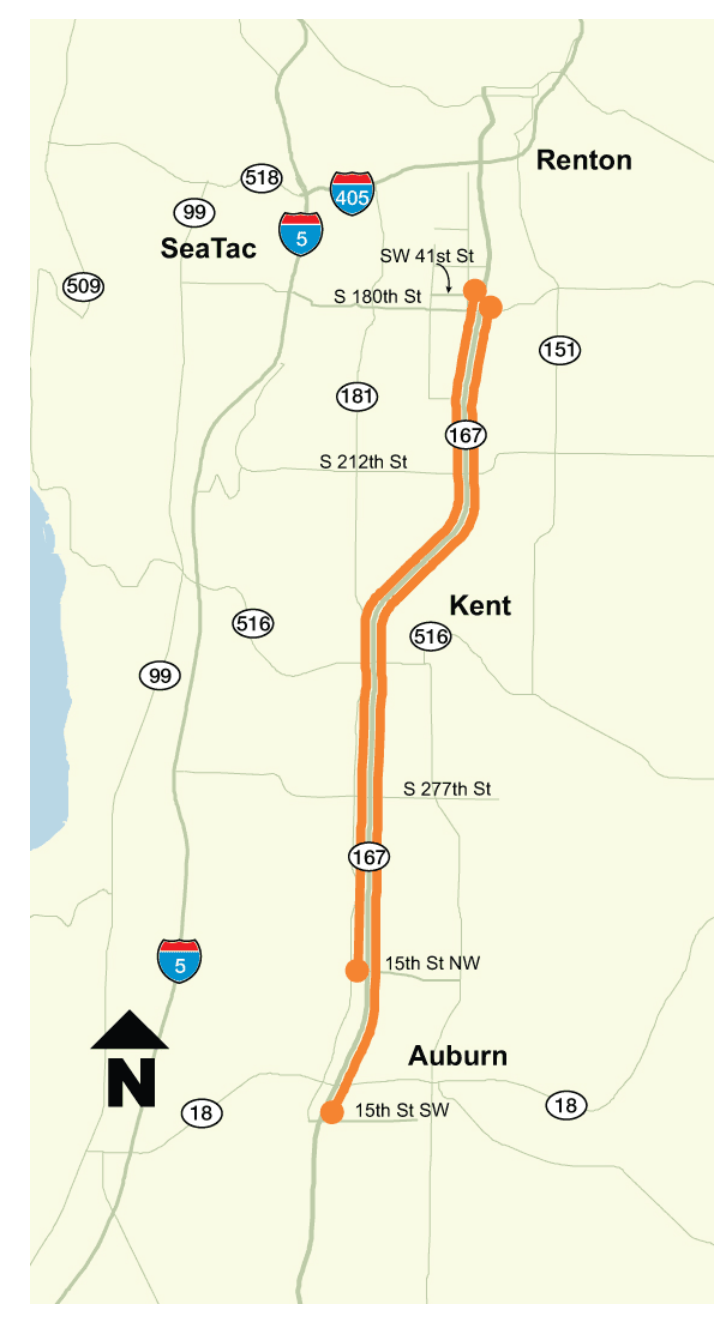

Figure 14 Location of SR 167 HOT Lanes 


\section{Previous HOV Lanes Operations}

Previously there was one HOV lane in each direction with free access to two or more person carpools and vanpools. The HOV lanes were separated from the GPLs with a single white line and carpoolers could access the lanes from anywhere along the length of the lane.

\section{Why Were HOT Lanes Considered?}

Over the last decade, congestion in the central Puget Sound region steadily worsened. Over 30 percent of the central Puget Sound's core freeway system is congested during peak periods. It is estimated that by the year 2030, over 45 percent of the core freeway system will be congested. The region has an extensive network of freeway HOV lanes - totaling over 200 lane miles. HOV systems at several key locations in the region effectively move almost a third of total person trips in about 16 percent of the total vehicles. However, there has been a public perception that some HOV lanes are "empty" lanes prompting several initiatives to convert existing HOV lanes to GPLs (WSDOT, 2005).

In response to the increasing congestion in the region and the HOV concerns, WSDOT evaluated the HOT lane concept as a means to improve traffic management while maintaining the efficiency of the region's HOV system operation. WSDOT evaluated the major HOV corridors in the central Puget Sound region as potential locations for the HOT lane pilot project. The two primary criteria used to evaluate each highway were significant peak period congestion and substantial available HOV capacity. An average travel speed of less than $60 \mathrm{mph}$ on the GPLs was used as a surrogate for the congestion evaluation criteria. An average HOV capacity of 1500 vehicles per hour per lane (vphpl) was used as the HOV capacity evaluation criteria. This 1500 vphpl threshold was used to determine if the HOT lane could generate enough revenue to support itself at this minimum level of vehicle throughput.

Five corridors with both substantial congestion levels and surplus HOV capacity were selected as finalists for the pilot project. Of the five corridors, SR 167 was selected as the top candidate for a proposed HOT lane pilot project because the corridor:

- has congestion in the peak direction on the GPLs;

- has available HOV lane capacity; and 
- has room to make needed improvements (WSDOT, 2005).

In comparison to the other candidate corridors, the SR $167 \mathrm{HOV}$ lane segment had the highest unused HOV capacity combined with substantial GP speed reductions and a consistent length of unused HOV capacity. SR 167 experiences strong peak direction congestion during both the morning and evening peak periods and carries approximately 120,000 vehicles a day. During the peak hours the speed reductions reach 15 to $25 \mathrm{mph}$ in the GPLs. Morning congestion occurs in the northbound direction as SR 167 approaches I-405 in Renton. Afternoon southbound congestion occurs primarily as SR 167 travelers approach SR 18 in Auburn (see Figure 14). During peak periods, neither northbound nor southbound HOV lanes operate at capacity leaving significant space for more vehicles in both. Based on a threshold of 1500 vehicles per hour, there was an available capacity for at least 450 more vehicles in SR 167's HOV lane (WSDOT, 2003).

\section{Objectives of the HOT Lanes}

The primary goal of the SR 167 HOT lane project was to test the viability of the HOT lane concept and determine if and how HOT lanes could be implemented on other highways in the central Puget Sound region. The project objectives included (WSDOT, 2005):

- "Testing the HOT lane concept's ability to maintain the speed and reliability of the HOV system without adversely impacting congestion along the project corridor and/or the regional highway system.

- Testing the ability of a HOT lane to generate a stream of revenue that can be used to pay for the operation and maintenance of the facility as well as transportation system improvements.

- Assessing the level of public interest and support for the HOT lane concept.

- Collecting performance data to help determine if HOT lanes could be used effectively in other locations and what modifications would be necessary to help ensure their successful implementation.

- Assessing the socio-economic impacts of the facility".

In Seattle, one of the primary equity concerns for the SR 167 HOV-to-HOT lane conversion pilot project was transit. Advocacy groups stressed that the minimum toll on the new HOT lane facility should be no lower than the transit fare in the same corridor. Since too low a toll will 
lower the out-of- pocket costs and make trips quicker for SOV commuters than transit users, a factor that could convert transit riders into solo drivers (Weinstein and Sciara, 2004).With no limit on the use of HOT lanes by the SOVs, the HOT lanes might eventually become overcrowded. As a result a bill was passed with following changes to make HOT lanes work better (Transportation Choices Coalition website):

- WSDOT was required to change the price to enter the HOT lane "dynamically," to ensure that single occupant vehicles don't cause congestion in the HOT lane. Toll rates are raised significantly as traffic worsens; ensuring that buses and carpools can keep moving at a good speed.

- A share of revenue collected from HOT lanes dedicated to improve transit, vanpool, rideshare and trip reduction services in the corridor. This is key to spreading the benefit of HOT lanes to all types of travelers.

\section{Change in Operations Due to HOT Lanes}

Previously carpoolers could enter and leave the lane along its entire length and the lane was separated by a single white line from the GPLs. With the HOT lane concept, motorists can enter and exit the HOT lanes at designated access points providing more predictable entry and exit maneuvers. Drivers can enter at the beginning of the HOT lanes and at several 0.5-mile-long access zones. There are six northbound and four southbound access zones marked with single dashed lines. Access points are located between interchanges. The access point design is based on I-394 diamond lane section of the HOT lane project in operation in Minnesota (SR 167 HOT lanes website). Other than at entry/exit points, the HOT lanes are separated from GPLs by double white lines which are illegal to cross and the buffer is 4 feet wide for added safety. There is also a 10 feet inside shoulder is also available. To provide the 10 foot shoulder and 4 foot buffer, the corridor was re-striped and the GPLs were made 11 foot and 12 foot wide.

\section{Impact on Transit}

There were seven buses using the HOV lane during the peak period in the peak direction. With HOT lanes the only service change in bus operations was the slight modification of the two routes (564 and 565). Transit officials fine-tuned the route alignments, directing buses to enter 
SR 167 at SR 516 instead of 84th Avenue. The adjustment allowed the buses to take better advantage of the HOT lanes' access zones. (WSDOT, 2009). All movements in and out of the HOT lane were also restricted to designated access points where there are openings in the buffer.

Comparing the ridership before and after HOT lanes: Following are the average weekday transit ridership (Table 20) for the SR 167 HOT Lanes Pilot Project (May 2007 - March 2008 compared to May 2008 - March 2009). There are two bus routes (564 and 565) that use the corridor and a parallel heavy rail service (South Sounder). It can be observed from the table that transit ridership increased by more than 15 percent for both express bus routes as well as rail.

\section{Table 20 Average Weekday Ridership for Express Lanes Bus Routes (Source: Sound Transit, Tyler Petterson, WSDOT)}

\begin{tabular}{|l|c|c|c|}
\hline Bus Route & $\begin{array}{c}\text { Before opening } \\
(\mathbf{2 0 0 7}-\mathbf{2 0 0 8})\end{array}$ & $\begin{array}{c}\text { After opening } \\
\mathbf{( 2 0 0 8}-\mathbf{2 0 0 9 )}\end{array}$ & $\begin{array}{c}\text { Percent } \\
\text { Change }\end{array}$ \\
\hline Route 564 & 1,280 & 1,530 & $19 \%$ \\
\hline Route 565 & 1,570 & 1,800 & $15 \%$ \\
\hline South Sounder & 7,750 & 9,000 & $17 \%$ \\
\hline
\end{tabular}

However this increase in transit ridership along the HOT lane corridor was found to be similar to the overall increase in regional transit ridership. During the period 2005-2008 transit ridership in the region grew a total of 23 percent, outpacing a strong national increase in transit ridership of 10 percent. This increase in transit ridership was attributed to the large increase in gasoline prices (Puget Sound trends, 2009).

To further investigate the transit ridership over the time, average weekday ridership for May through October for the years 2007 through 2009 was examined (see Figure 15). This provides an opportunity to compare bus ridership for the same period of year (May through October) before the HOT lanes, the year HOT lanes started, and one year after the HOT lanes. It can be seen that after the increase in bus ridership in 2008, ridership decreased in 2009. This decrease in ridership in 2009 was attributed to the reduction in gas prices, economic stability and regional job loss (WSDOT, 2010). 


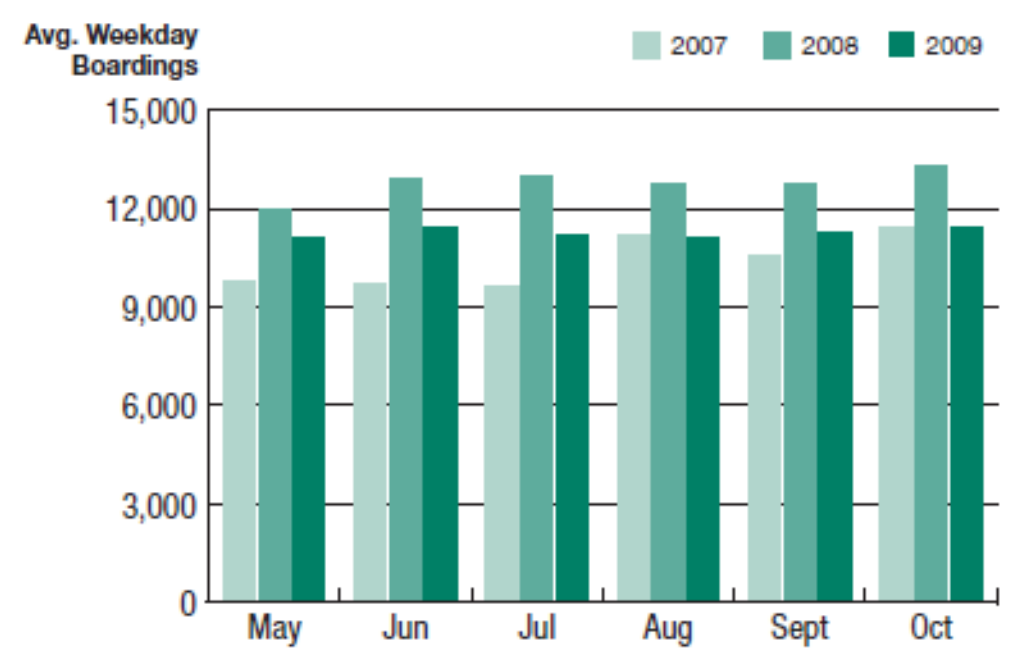

Figure 15 Average Weekday Ridership of the SR 167 Corridor (WSDOT, 2010)

When ridership increased in 2008, it was assumed to be (WSDOT, 2009) one of the factors (along with rising gas prices, and the economic downturn) for the drop in corridor volume. In the first year of HOT lanes operation (May 2008 through April 2009), average daily traffic volumes on SR 167 declined roughly 2 percent. Declining roadway volumes were consistent with regional and national trends; however traffic volumes in April 2009 returned to April 2008 levels.

Since, the increase in bus ridership occurred around the time of opening of SR 167 HOT lanes, there could be a possibility that HOT lanes were a factor in this ridership change. However, with HOT lanes, the only service changes of the transit operation was the fine tuning of the bus routes to help them make better use of HOT lanes. Plus there was a small increment in speed of HOT lanes (7 percent) (WSDOT, 2010). It would seem unlikely that these small changes would cause a 15 percent increase in ridership.

The above findings show that the increase in bus ridership in 2008 and then the subsequent decrease in 2009 could have been largely due to gas price fluctuation (increasing in 2008 and decreasing in 2009) (WSDOT, 2010) and not due to HOT lanes. The relation between gas prices and bus ridership will be discussed in a later section in detail. 


\section{Impact on Carpooling}

Surveys were conducted in August 2008 and May 2009 (both after the HOT lanes opened) in which respondents were all Good To Go! (name of electronic tolling program) transponder owners who paid to use the HOT lanes at least once. The survey data was sent by Tyler Petterson, WSDOT-Tolling Division. In the 2009 survey, there were survey respondents who were wrongly charged the tolls when they were actually carpooling on HOT lanes and hence were selected for the survey. This happened with the customers who purchased the transponder before May 3, 2008 when shields were not available to keep them from being charged the toll when carpooling. However, this problem was resolved for the transponders sold after May 3, 2008. Therefore, for the purpose of this analysis only those respondents who bought their transponders after May 3, 2008 were considered (respondents in the survey were asked the day of their purchase of transponder).

This problem of improperly charging HOVs a toll and including them in the survey might also have occurred in the August 2008 survey. But in the survey, respondents were not asked their date of purchase of the transponder, therefore, for the purpose of this research, only the May 2009 survey will be examined.

Respondents were asked their mode of travel when they were not paying users of HOT lanes. The respondents could select more than one mode and they were divided (see table 6) among those who mentioned (a) drive alone on GPLs as their only mode, (b) carpool/vanpool as their only mode and (c) drive alone as well as carpool (see Table 21).

Table 21 Mode of Survey Respondents

\begin{tabular}{|l|c|}
\hline Mode used when not a paying HOT lane user & $\begin{array}{c}\text { May 2009 } \\
(\mathbf{N}=\mathbf{9 7 5})\end{array}$ \\
\hline Drive alone on GPL only & $47 \%$ \\
\hline Drive alone or carpool & $26 \%$ \\
\hline Carpool/Vanpool only & $10 \%$ \\
\hline
\end{tabular}


Almost half of the respondents (47 percent) exclusively drive alone on GPLs when not using the HOT lanes as a paying customer. Only one-tenth of the respondents exclusively carpool when not paying to use the HOT lanes.

In the May 2009 survey, respondents were also asked the frequency of using the toll option and carpooling in the HOT lanes. The questions were "On average how often do you pay to use the HOT lanes?" and "On average how often do you travel HOT lanes as carpool?" The results of the two questions with respondents divided in to the modes they use when not traveling HOT lanes as paying users are found in Table 22.

Table 22 Frequency of HOT Lane Mode Usage

\begin{tabular}{|c|c|c|c|}
\hline & \multicolumn{3}{|c|}{ Mode used when not a Paying HOT lane customer } \\
\hline & $\begin{array}{c}\text { Drive alone } \\
\text { only } \\
(N=459)\end{array}$ & $\begin{array}{c}\text { Drive alone } \\
\text { or carpool } \\
(N=256)\end{array}$ & $\begin{array}{l}\text { Carpool/Vanpool } \\
\text { only }(\mathrm{N}=94)\end{array}$ \\
\hline \multicolumn{4}{|c|}{ How often do you use the HOT lanes as a Paying HOT lane } \\
\hline $\begin{array}{l}\text { more than } 4 \text { times per } \\
\text { week }\end{array}$ & $22.7 \%$ & $23.8 \%$ & $14.9 \%$ \\
\hline 2-3 times per week & $18.3 \%$ & $19.1 \%$ & $14.9 \%$ \\
\hline 1 time per week & $9.8 \%$ & $13.3 \%$ & $10.6 \%$ \\
\hline $\begin{array}{l}\text { once a week or more } \\
\text { (total of above three) }\end{array}$ & $50.8 \%$ & $56.3 \%$ & $40.4 \%$ \\
\hline \multicolumn{4}{|c|}{ How often do you use the HOT lanes as a carpool in HOT lane } \\
\hline $\begin{array}{l}\text { more than } 4 \text { times per } \\
\text { week }\end{array}$ & $1.5 \%$ & $7.4 \%$ & $18.1 \%$ \\
\hline 2-3 times per week & $4.4 \%$ & $17.6 \%$ & $11.7 \%$ \\
\hline 1 time per week & $7.4 \%$ & $18.8 \%$ & $16.0 \%$ \\
\hline $\begin{array}{l}\text { once a week or more } \\
\text { (total of above three) }\end{array}$ & $13.3 \%$ & $43.8 \%$ & $45.7 \%$ \\
\hline
\end{tabular}

In Table 7, for drive alone only respondents, the percentage of respondents using the HOT lanes as carpool should be zero (since these are the respondents who mentioned drive alone as their only mode when not paying users of HOT lanes). This might have been because of some respondents' misunderstanding the question -"How have you traveled on SR 167 when not a paying HOT lane customer?" However, it can be seen that the majority of respondents use the HOT lanes as toll users at least once a week. 
The most surprising result was from the respondents who mentioned carpool as their only mode when not using the HOT lanes as a paying user. Almost as many respondents mentioned they used the express lanes as a paying user (40 percent) as they carpooled to use the lanes (46 percent). However, among the three groups, carpoolers used the HOT lanes as paying users the least frequently.

As noted in Table 6, only one-tenth of the respondents mentioned carpool as their only mode when not using the HOT lanes as paying users. Therefore, even though these respondents have higher frequency of using toll option on HOT lanes (40 percent using it at least once a week) it will have a minimal overall impact on mode shift. It should also be noted that all these respondents are transponder owners and therefore, expected to use the HOT lanes as paying users.

\section{Relationship Between Gas Prices, Tolled Trips and Transit Usage}

In the previous section, how transit ridership varied with gas prices was briefly examined. This section will examine this issue in detail to understand any impact on transit due to the HOT lanes. The SR 167 HOT lanes began in May 2008 approximately when gas prices reached their peak of \$4 (see Figure 16). 


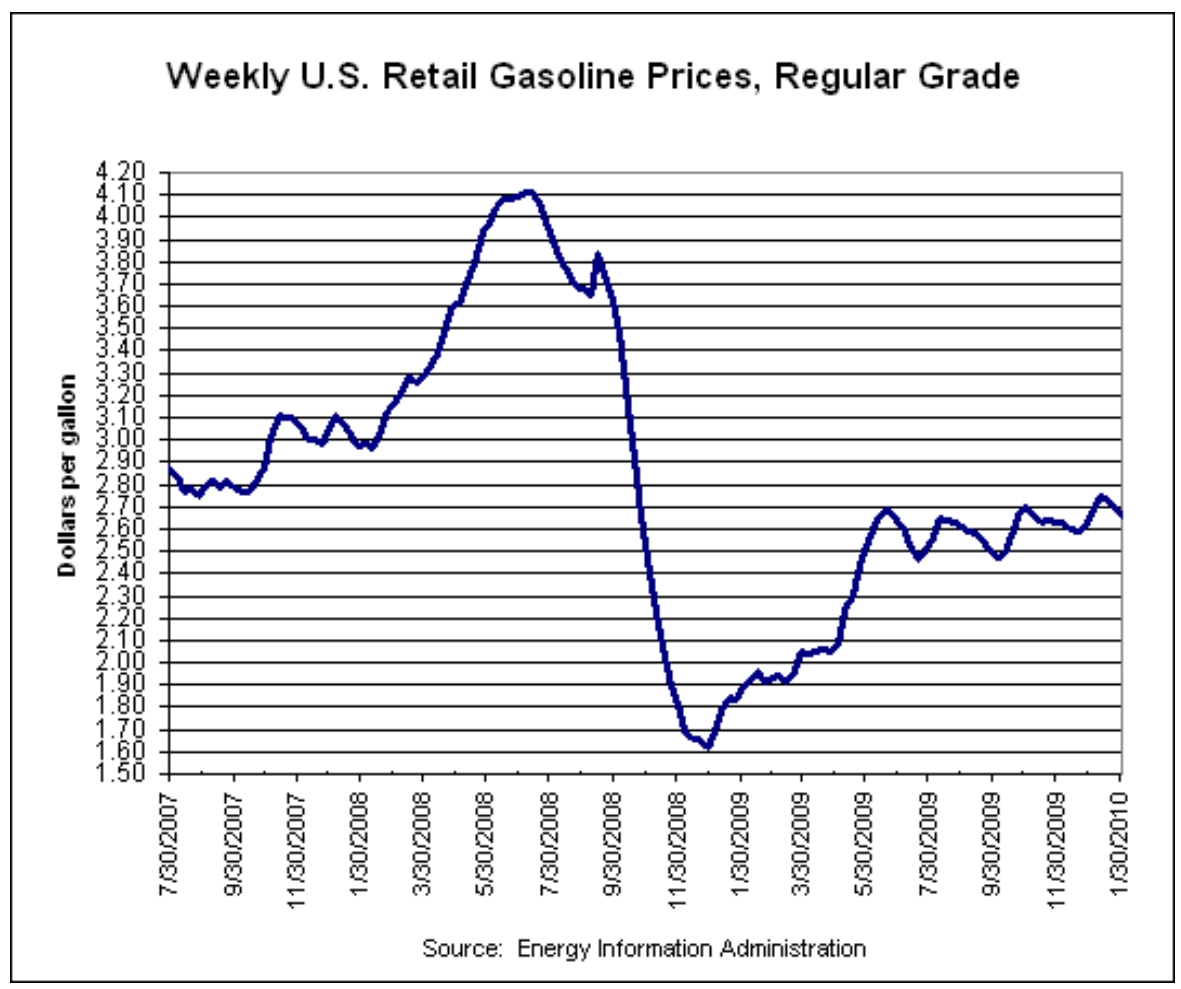

Figure 16 Weekly US Retail Gasoline Prices (EIA website)

Following are the different changes which occurred from 2007 through 2009-

1. Bus ridership increased in the corridor (17 percent) as well as at the regional level (23 percent) from 2007 to 2008 due to increase in gas prices (Puget Sound Trends, 2009) and then decreased again in 2009 due to reduction in gas prices (see Figure 17).

2. Compared with 2008 there was an increase of 46 percent in the average daily tolled trips in 2009 (see Figure 18).

From the above two observations, when bus ridership decreased from 2008 to 2009, the tolled trips on HOT lanes increased by 46 percent during the same period. Also, during this period the gas prices also reduced (see figure6). This indicates that with decreasing gas prices, transit riders might have shifted to auto mode in GPLs as well as HOT lanes. 


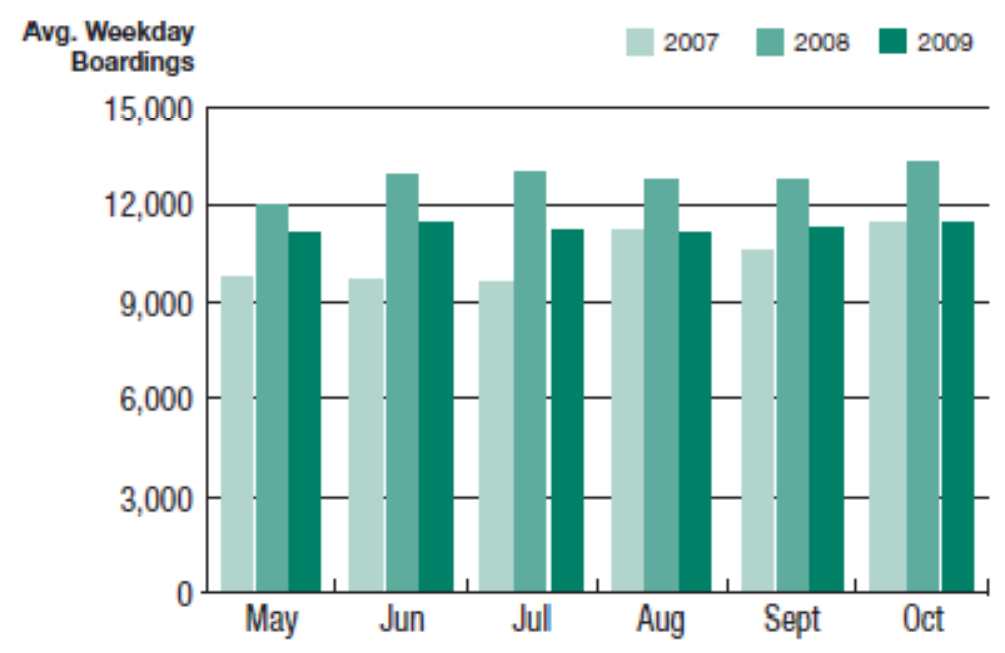

Figure 17 Weekday Transit Ridership (WSDOT. 2010)

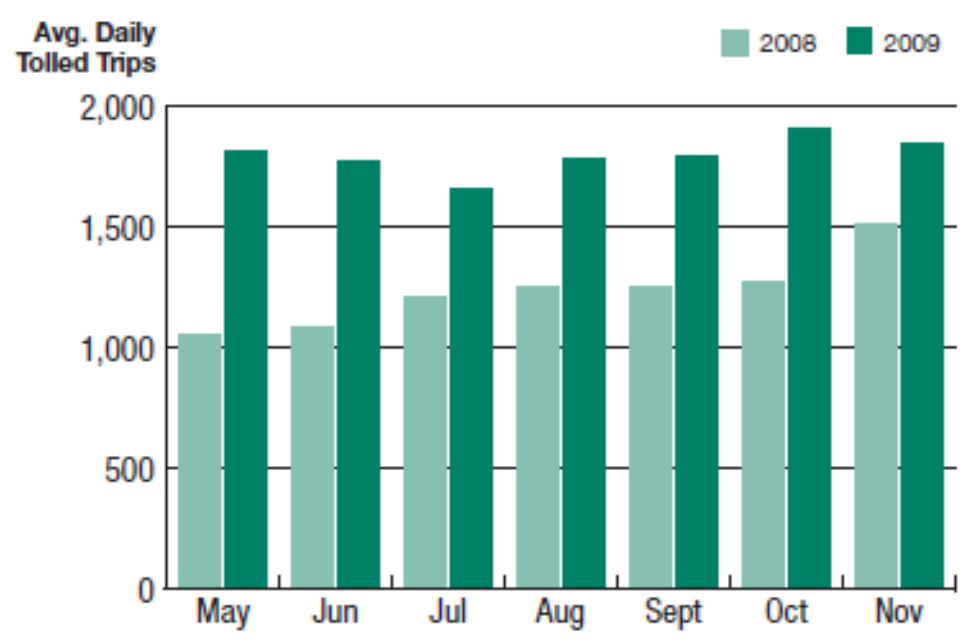

Figure 18 SR 167 HOT Lanes Average Daily Tolled Trips (WSDOT, 2010)

Therefore, the relationship between the HOT lane usage (toll as well as carpool) and gas prices was further examined. Northbound usage of HOT lanes from May 2008 through April 2009 (compiled from the performance reports and carpool data received by Kevin C. Beireis, WSDOT - Toll Division) and monthly average gas prices in Seattle (EIA website) are compiled in Figure 19. From the figure it can be observed that there is a strong positive correlation between carpool usage on the HOT lanes and gas prices. 
From October 2008 to November 2008, the toll usage almost doubled from 710 to 1510 toll users per weekday. While the carpool usage decreased by around 1500 vehicles between the two months. Note that gas prices decreased by almost 90 cents from October to November, the biggest one-month drop in gas prices. This strongly implies that the reduction in gas prices results in carpools breaking up and transit riders leaving the bus (as noted above). While the carpools can shift to SOVs on HOT lanes and/ or GPLs, bus riders can shift to these two as well as carpool in HOT lanes. The other possible explanations for the decreasing carpool- as shift to carpoolers on GPLs or to transit would not occur due to a drop in gas prices.

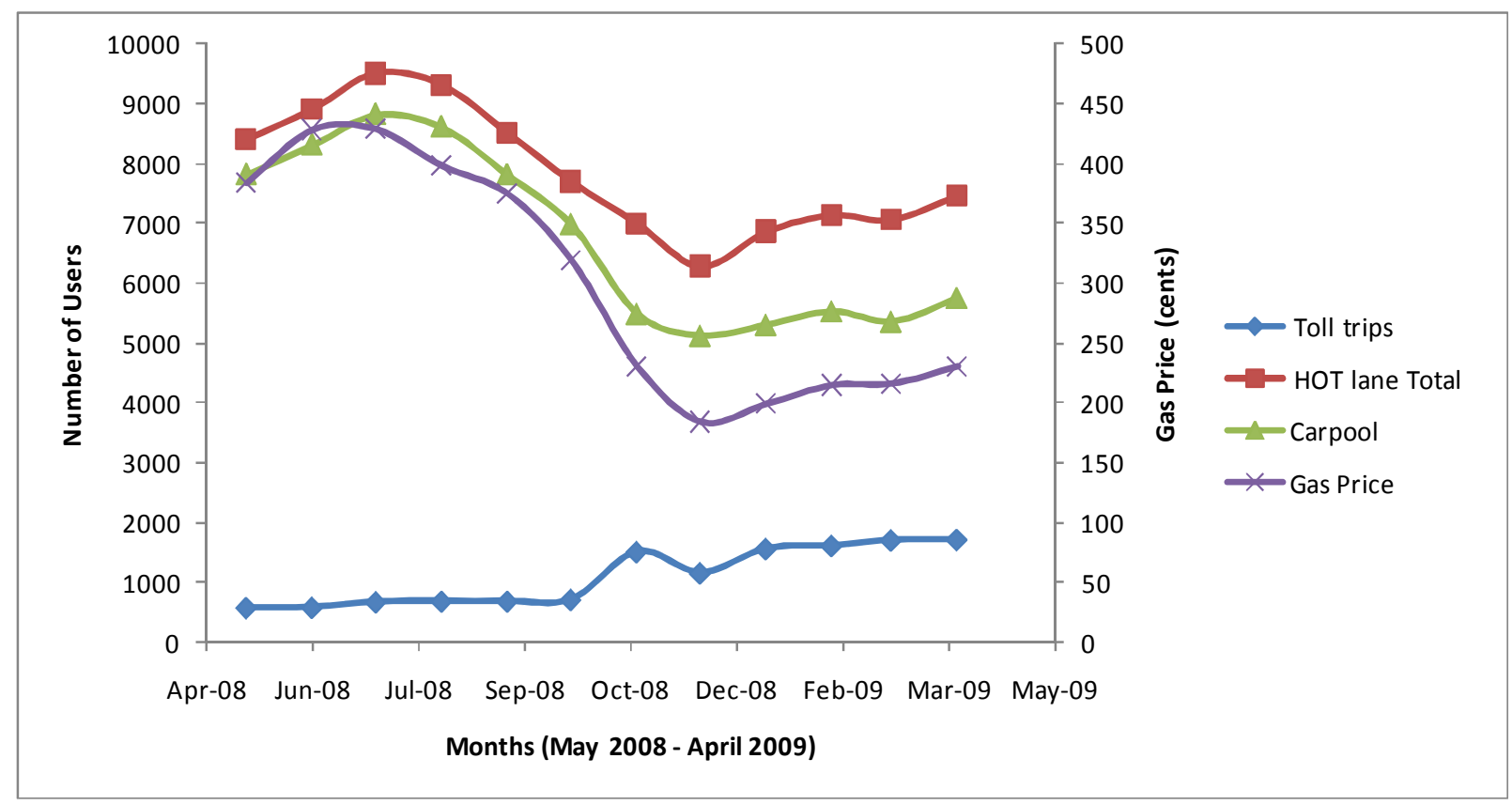

Figure 19 Usage of HOT lanes by Toll and HOV Users and Gas Prices for May 2008 Through April 2009

The above findings provide some insight into the impact of gas prices on the mode choice of travelers. An increase in gas price makes carpooling more attractive by either splitting the cost of auto expenses or by saving the toll if the traveler was previously an SOV on HOT lanes. Therefore, the usage of HOT lanes, to some extent, is dependent on exogenous factors like gas prices which is generally not included as one of the elements in the planning of HOT lanes. It also implies that, the performance of HOT lanes should be judged in light of the gas prices in the 
respective locations and it becomes even more crucial when the two HOT lanes are compared based on their usage.

It should be noted that the trend of toll usage of HOT lanes is almost flat. Also, the only jump occurred coincided with a high reduction in gas prices. One of the reasons for the low usage could be the economic recession as noticed for the I-25 Express Lanes leading to increased unemployment rate from early 2008.

\section{Usage of the HOT Lanes}

For the first six months of HOT lane operation (May- November 2008) the average weekday tolled trips in HOT lanes was 1230. During the same months for 2009 there was an average of 1795 trips, an increase of 46 percent (see Figure 8). Total corridor volume increased by 3 percent during this time. In one year, northbound weekday usage of carpools decreased by more than 25 percent (from 7800 to 5700) while the toll paying SOVs increased by almost 300 percent (from 580 to 1700$)$.

\section{Safety Implications of the HOT Lanes}

In the May 2009 survey more than one-third of the respondents agreed with the statement that HOT lanes improved roadway and safety. In a focus group (WSDOT, 2009a) conducted by WSDOT in January 2009, participants were concerned about safety and enforcement. There were complaints about getting cut off as they exit the HOT lanes, carpoolers thinking that they are exempt from the double white line crossing and insufficient enforcement. Participants were also concerned regarding the speed difference between the HOT and GPLs. There were also complaints about insufficient time to exit the freeway after crossing the dotted white lines, not enough space to enter/exit at the dotted white lines, and not enough places where the dotted white lines can be crossed. However, WSDOT remains confident (WSDOT, 2009) that the SR 167 HOT lanes are not adversely impacting the safety in the corridor but no conclusions regarding the safety impact of HOT lanes have been made yet due to insufficient data. 


\section{Travel Time}

During the first year of HOT lane operations, travel speeds on the HOT lanes were at least 45 mph during the AM peak (7 AM- 8 AM) and PM peak (5 PM-6 PM) for 99 percent of the time. The northbound HOT lane provided weekday drivers with an average time savings of eight minutes in the peak-hour for an average toll of $\$ 1$. The weekday southbound HOT lane provided drivers with an average savings of four minutes during the peak-hour for an average toll of $\$ 1$.

\section{SR 167 HOT Lanes and I-394 Diamond Section}

There are many similarities between the SR 167 HOT lanes and the diamond lane section of the I-394 HOT lanes. Both are concurrent, one lane each direction alongside 2 GPLs in each direction (as an exception, I-394 has one auxiliary lane in westbound direction in addition to the 2 GPLs). The HOT lanes are separated from the GPLs using double white lines. Also, both HOT lanes have dynamic pricing with designated intermediate access points. These similarities between the two HOT lanes help to avoid many exogenous factors and provide an opportunity to compare different impacts of the two HOT lanes.

However, the two HOT lanes differ on some design and operational issues. The buffer between HOT lanes and GPLs for I-394 diamond section is (Congressional Budget Office website) 2 feet while SR 167 buffer is 4 feet wide. Also, the SR 167 HOT lanes operate from 5 AM to 7 PM in each direction while I-394 diamond lanes have peak hour operations (6 AM to 10 AM and 2 PM to $7 \mathrm{PM}$ ). In the off -peak hours the I-394 HOV lane is used by general traffic while the I-394 HOV lane in other direction operates as HOT lane. ADT of the two corridors at the time of HOT lane implementation was 120,000 for SR 167 in 2008 and 148,200 for I-394 in 2005. Before the SR 167 HOT lanes, the speed in the GPLs dropped to $35 \mathrm{mph}$. However, before the I-394 Express Lanes, travel speed at different sections along the diamond section was above $50 \mathrm{mph}$ except at Xenia Avenue section (see figure 2) where it dropped to $43 \mathrm{mph}$ in the PM peak. 


\section{Usage of HOT lanes}

When SR 167 started operation in May 2008, the gas prices reached their peak (\$4). When I-394 HOT lanes started operation in May 2005, gas prices were \$1.95 a gallon and reached over \$3.00 a gallon by July 2006 (Cambridge Systematics, 2006a).

To make a comparison between the usage of two HOT lanes, without any bias due to gas price historical gas prices were observed (EUA, website) in Minnesota and Seattle and it was found that gas prices in Minnesota in the first quarter of year 2007 matches with those in the Seattle in first quarter of 2009 (15- 30 cents less than \$2.50). Therefore, the usage of two HOT lanes will be compared during these two periods first quarter of 2007 for I-394 and first quarter of 2009 for SR 167.

Table 23 shows the usage of two HOT lanes by toll users as well as carpoolers (including vanpools and buses) and the GPL volume in the AM and PM peak periods. For the I-394 the data is from January -April 2007 (MnDOT, 2008) and for SR 167 the data is from January-April 2009 (carpool data obtained from WSDOT) (the percentage of volume of toll and HOV usage to the total volume of corridor is given in parentheses).

Table 23 Toll and HOV Usage of HOT Lanes and GPL Volume

\begin{tabular}{|l|c|c|c|c|c|c|}
\hline \multirow{2}{*}{ Peak Period } & \multicolumn{2}{|c|}{ Toll Usage } & \multicolumn{2}{c|}{ HOV Usage } & \multicolumn{2}{c|}{ GPL } \\
\cline { 2 - 7 } & I-394** & SR 167 & I-394 & SR 167 & I-394 & SR 167 \\
\hline 7 - 8 AM & $360(7 \%)$ & $250(6 \%)$ & $744(13 \%)$ & $690(18 \%)$ & $3921(80 \%)$ & $3200(76 \%)$ \\
\hline 5 - 6 PM & $310(5 \%)$ & $155(4 \%)$ & $474(6 \%)$ & $760(21 \%)$ & $5572 *(89 \%)$ & $3000(75 \%)$ \\
\hline
\end{tabular}

*One additional auxiliary lane **The AM peak counts for I-394 diamond lane section are from Louisiana Avenue section and the PM peak counts are from the Winnetka Avenue section.

From the above table, it can be observed that HOVs make larger percentages of travelers on SR 167. Since GPLs in SR 167 were more congested than in I-394 as mentioned above, therefore carpoolers may have more incentives to carpool in SR 167 than I-394. This might have resulted in higher percentages of carpool on SR 167. 


\section{Safety Implications of the HOT lanes}

The planners of SR 167 HOT lanes based the separation mechanism and design of access points of the SR 167 HOT lanes on the diamond lane section of I-394 (SR 167 HOT lanes website).

\section{Enforcement Efforts by the HOT Lanes}

The MnPASS project also involved funding the enforcement activities for the HOT lanes. Between May and December 2005 (first 8 months of HOT lane operations), public safety officers made nearly 5,000 stops on the I-394 corridor. Approximately 50 percent of these stops involved HOV violations or motorists illegally crossing the double white lines. This increased enforcement effort apparently had a positive impact on compliance as HOV violation rates decreased compared with conditions in the years prior to MnPASS, and the violation rates in the I-394 corridor were significantly lower than on non-MnPASS equipped HOV facilities on I-35W (Cambridge Systematics, 2006a).

Similar to MnPASS, as a part of the SR 167 HOT Lanes Pilot Project, the Washington State Patrol (WSP) is funded from the HOT lane operations to provide additional enforcement on HOT lanes. During first year of operations (May 2008- April 2009), there were 4,317 traffic stops, yielding 2,054 citations for HOV/HOT violations and 328 citations for crossing the double white line that separates the HOT lanes from the GP lanes. WSP officials said "they are encouraged by the compliance rate, which is estimated to be 95 to 97 percent" (WSDOT, 2009).

\section{Difference in Safety Perceptions of the HOT Lane Users}

In an attitudinal panel survey conducted in spring 2006, 91 percent of I-394 HOT lane subscribers were satisfied with the ease of identifying the MnPASS entry points and 78 percent were satisfied with the safety of merging into the MnPASS lanes.

As opposed to this, SR 167 HOT lane focus group participants (January 2009) were concerned regarding the safety of getting in and out the HOT lanes and 23 out of 35 participants mentioning problems with double white lines- including: getting stuck behind slow drivers, drivers illegally crossing the double white lines, and limited access to enter and exit the lanes. However, because 
of the difference in the two approaches- panel survey in I-394 and focus group in SR 167, no direct comparison could be derived. 


\section{CHAPTER VI}

\section{I-10 AND US 290 HOT LANES, HOUSTON}

(Note: This research focuses on Katy Freeway HOT lanes as they existed from 1998 to 2008. The new Katy Freeway Managed lanes opened very recently (April 2009) and data on their usage was not available).

\section{Introduction}

The QuickRide program started in January 1998 on Katy freeway (I-10) and in November 2000 on Northwest freeway (US 290). The program allows the two-person carpool to use the HOV lanes for a fixed fee of $\$ 2.00$ per trip for limited time periods. These HOT lanes are the only HOT lane projects which do not allow access to the SOVs. And unlike all other lanes the toll for HOV2 is a flat per trip fee. Therefore, these HOT lanes have not been compared to any other existing HOT lane.

The Katy HOT lane is 13.3 miles long, single reversible lane (except for a short 2-lane segment near the eastern end) and barrier separated from the GPLs (see Figure 20). The lane is 19 feet wide or wider in most locations. The time period for HOV2 pricing is limited to 6:45 AM to 8:00 AM and from 5:00 PM to 6:00 PM and HOV2s may use the facility free of charge outside of these periods. HOV3+ can use the lanes for free at all times.

Access Points: Slip ramps are available directly on to the HOT lanes at the western end, near the middle (near Gessner road), and at the eastern end (eastern extension) and a T-ramp connected to the Addicks park and ride lot. Direct access is available to surface streets at Post Oak Road. Most enforcement occurs on the eastern end where there is more room to pull over vehicles.

The Northwest freeway HOT lane is 13.5 miles long, single reversible lane (except near the Southeastern end - between Dacoma and Northwest Transit Center- where there is one lane per direction) (see Figure 20). Similar to the Katy HOT lane, this is also barrier separated from the GPLs. However, HOV2 pricing on this HOT lane is only from 6:45AM to 8:00AM, when the facility serves inbound traffic. HOV3+ can use the lane for free at all times. 
Access Points: A Slip ramp provides access directly on to the HOT lane at the northwestern end and T-ramps to the Northwest Station, West Little York, Pinemont and Northwest Transit Center park and ride lots. Wishbone ramps provide access to the frontage road near Dacoma Road. Most enforcement occurs on the eastern end where there is room to pull vehicles over. However, sometimes motorcycle officers will monitor the lane from the T-Ramps.

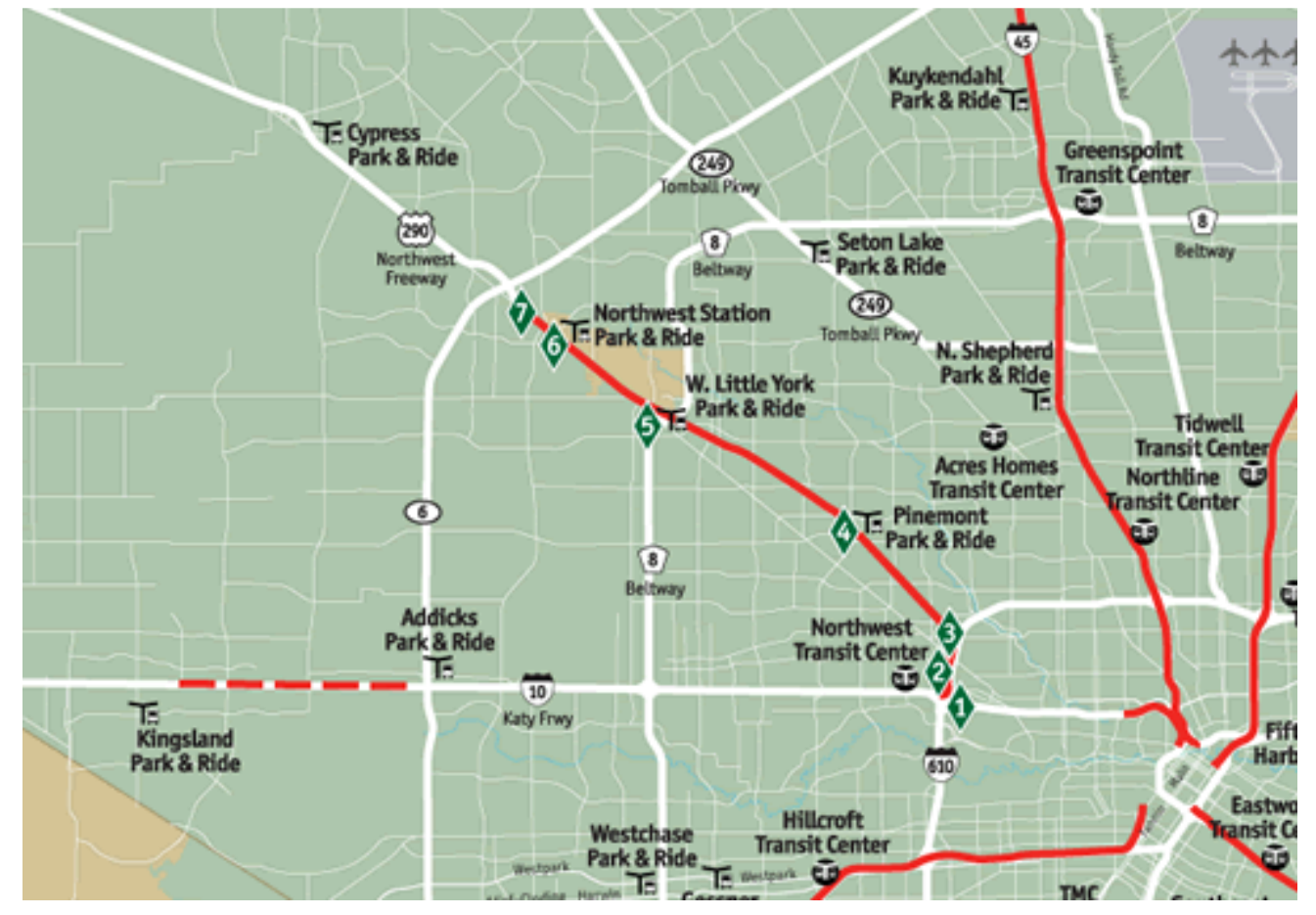

Figure 20 Location of US 290 and I-10 (source: METRO website)

\section{Why Were HOT Lanes Considered?}

The following section describes the different conditions in the I-10 and US 290 corridors which lead to the development of HOT lanes.

\section{Katy Freeway HOT Lane}

Designed to carry 79,200 vehicles per day, the Katy Freeway carried over 207,000 vehicles per day, and is considered one of the most congested stretches of freeway in Texas in early 2000s. The freeway also has the highest daily truck volumes of any roadway in the state. Traffic generated from six radial highways, nine employment centers, the Port of Houston, and through 
truck traffic are all compressed into three lanes in each direction. (National Transportation Library (NTL) report)

When the Katy HOV lane opened in 1984, only transit buses and registered vanpools could use the lane. To make better use of this road capacity, the restrictions were relaxed in stages until any vehicles with two or more occupants (HOV2+) were allowed. The lane soon became congested during peak traffic periods due to the high number of carpool vehicles using the lane. Prompted by this, Houston METRO (transit agency responsible for the operation of the HOV lanes) along with TxDOT, restricted usage of HOV lanes to HOV3+ during the morning peak period (6:45 a.m. to 8:15 a.m.) in 1988. The time period was later changed to 6:45 AM to 8:00 AM in 1990. Soon after, HOV3+ restriction was also extended to during the afternoon peak period (5:00 PM to 6:00 PM) because of increased congestion.

As a consequence, these occupancy restrictions (HOV3+) resulted in a considerable reduction in peak period traffic and available capacity in the HOV lanes. Also, the number of persons moved by the lane during the peak hour declined by 30 percent. However, less onerous restrictions (HOV2+) had resulted in excess demand and congestion on the lanes. As a solution, the QuickRide program was created allowing HOV2s to use the lanes for a price during the peak periods. This would limit demand to an acceptable level, make more efficient use of the lane, and provide a revenue source to help pay for the program.

\section{Reasons SOVs were Not Allowed on the HOT Lanes}

QuickRide operators decided SOVs would not be allowed to use the lane (even if willing to pay the toll) because of corridor's high travel demand and its limited capacity (one reversible lane), as well as SOV use restrictions tied to the HOV lane's original construction financing from the Federal Transit Administration (FTA).

Unless the fee for SOVs was high enough to deter most of the SOVs from using the HOT lanes the admission of SOVs to QuickRide would have quickly congested the facility. Operators expected that the number of HOV2s that would take advantage of the peak hour pricing would still allow the lane to operate at free flow. 


\section{Estimation of Available Capacity}

The primary goal of pricing the HOV lane was the effective use of the Katy HOV lanes. To determine the additional number of toll paying HOV2 vehicles that could be provided access to HOV lanes, it was considered that the operations must not have an adverse impact on the operations of the HOV lanes or GPLs. Katy HOV lanes moved 34 percent of all the persons in the corridor during the AM and PM peak periods combined. Therefore, the potential benefits of pricing the lanes to a relatively modest number of travelers mush not impede the movement of existing HOV3+ vehicles. Also, an ideal capacity of 1200 vehicles per hour was considered to estimate the available capacity for the toll paying HOV2s. For each of the AM and PM peak period an additional capacity of 600 vehicles was estimated. However, the time of arrival of the additional vehicles was also crucial in maintaining the free flow conditions since the additional capacity was estimated by 150 vehicles in each quarter hour of the peak hour.. So, vehicles exceeding 150 in a quarter hour will degrade the operation (Stockton et al., 1998).

Before and after studies of the Katy showed that its HOT lane application had the following positive results (FHWA website):

- It increased the number of three-plus carpools during the peak;

- It redistributed two-plus carpools to before and after the peak hour;

- It increased average traffic speeds and improved the Katy HOV's level of service; and

- It transported the same number of passengers more efficiently.

\section{Northwest Freeway HOT Lane}

Through the 1990s, the Northwest freeway HOV lane use grows, and by 1998, the facility served 6,400 vehicles and 16,200 passengers per day. From September 1997 to April 1999, the lane witnessed a 37 percent increase in the number of peak hour vehicles. This rapid increase, particularly during the AM peak, caused operations to deteriorate. Average speeds in the Northwest HOV lane slowed to between $20 \mathrm{mph}$ and $30 \mathrm{mph}$ in the AM peak and the level-ofservice (LOS) reduced to "F." (FHWA website) 
Crowded HOV conditions also impacted buses and bus passengers using the facility. Buses serving the Northwest's park-and-ride facilities experienced on average 15-minutes of delay as well as increased operating expenses. Additionally, the large number of cars exiting the HOV facility at its terminus at the Northwest Transit Center negatively impacted the efficiency of bus movements and bus transfers that take place there. Commuters who arrive at park-and-ride lots along the facility and use buses on the Northwest HOV lane to reach downtown were particularly distressed. Commuter complaints to Metro noted deteriorating operations, delays, reliability problems, and lateness (FHWA website).

Due to the success of QuickRide on Katy freeway, Houston Metro considered HOV3+ operation similar to as a possible solution. In early 2000, Metro changed occupancy requirements on the Northwest HOV from two-plus to three-plus carpools from 6:45 to 8:00 AM. The facility experienced a noticeable drop in usage, alleviating crowding and restoring levels of service for transit users. In November 2000, QuickRide operations were launched on the Northwest Freeway (FHWA website).

\section{Objectives of the HOT Lanes}

The overall objectives of the QuickRide program were to (Shin and Hickman, 1999):

- Increase person-throughput in the Katy Freeway corridor during peak periods.

- Increase travel speeds on the GPLs during peak periods, assuming that many vehicles currently using the GPLs will divert to the HOV lane.

- Efficiently manage demand without adverse operating impacts on both the HOV lane and the GPLs.

\section{Impact on Transit}

A survey of Katy and Northwest freeway commuters (other than existing QuickRide users) was conducted in November 2003 to gather information about the commute travel patterns, socioeconomic characteristics, and opinions of proposed changes in the QuickRide program. Survey respondents were divided in to four groups- GPL travelers, HOV lane travelers, transit riders, and casual carpoolers (TTI, 2004). 
A total of 292 survey respondents made comments referencing METRO and among them almost half (42 percent) were complaints - 124 total (see Table 24). The majority of critical comments was specifically about afternoon peak period bus service- buses not operating on time and overcrowded buses. Complaints about afternoon bus service on the park \& ride routes represent 36 percent of the complaints about METRO. Other complaints were about park \& ride fares being too high for the quality of service, the condition of buses (either interior comfort or quality of maintenance), and opposition to intermediate stops at the Northwest Transit Center, the performance of drivers, and specific comments about problems getting home one evening after floods interrupted transit service (TTI, 2004).

Table 24 Complaints about METRO Service

\begin{tabular}{|l|c|c|c|c|c|}
\hline Complaints about METRO Service & GPLs & $\begin{array}{c}\text { HOV } \\
\text { lane }\end{array}$ & $\begin{array}{c}\text { Transit } \\
\text { Riders }\end{array}$ & $\begin{array}{c}\text { Casual } \\
\text { Carpools }\end{array}$ & $\begin{array}{c}\text { Total } \\
\text { Comments }\end{array}$ \\
\hline $\begin{array}{l}\text { Poor afternoon Park \& Ride } \\
\text { Service }\end{array}$ & $6 \%$ & $0 \%$ & $47 \%$ & $40 \%$ & $36 \%$ \\
\hline $\begin{array}{l}\text { General Complaints METRO } \\
\text { Service }\end{array}$ & $50 \%$ & $17 \%$ & $10 \%$ & $25 \%$ & $21 \%$ \\
\hline $\begin{array}{l}\text { Bus Fare Too High for Service } \\
\text { Quality }\end{array}$ & $33 \%$ & $33 \%$ & $5 \%$ & $10 \%$ & $12 \%$ \\
\hline Complaints About Buses & $6 \%$ & $17 \%$ & $12 \%$ & $13 \%$ & $11 \%$ \\
\hline Requests to Non Stop at NWTC & $0 \%$ & $17 \%$ & $10 \%$ & $10 \%$ & $9 \%$ \\
\hline $\begin{array}{l}\text { Complaints About Driver } \\
\text { Performance }\end{array}$ & $6 \%$ & $17 \%$ & $8 \%$ & $0 \%$ & $6 \%$ \\
\hline Service During Flood 11/17/2003 & $0 \%$ & $0 \%$ & $8 \%$ & $3 \%$ & $5 \%$ \\
\hline Total (N) & 18 & 6 & 60 & 40 & 124 \\
\hline
\end{tabular}

From the above findings of the complaints regarding the METRO transit there is little chances of any increase in ridership. Also, the HOT lane operation of QuickRide is only for the peak periods (6:45 AM to 8:00 AM and from 5:00 PM to 6:00 PM) therefore, the HOT lane operations are not significant enough to have any effects on transit. 
The above findings are also by examining the usage of QuickRide by transit users. In a survey of 185 QuickRide users in 1998 (Hickman et al., 2000), it was found that about 11 percent of the QuickRide trips were from the bus in the morning and about 5 percent of the trips in the evening. However, in actual numbers, this represented only about 15 trips per day.

\section{Impact on Carpooling}

\section{Previous Mode or Mode Used on Non-HOT Lane Trips of Those Using QuickRide}

A survey of 185 QuickRide (Hickman et al., 2000) enrollees was conducted shortly after the program began. Over half of the QuickRide trips were found to be SOVs moving into the HOV lane (51 percent in the morning, 58 percent in the evening). About one-quarter of the trips are two-person carpools moving from the main freeway lanes into the HOV lane (23 percent in the morning, 29 percent in the evening). In the morning, about 18 percent of QuickRide trips are diverted from higher occupancy modes, but in the evening only 1 percent represent diverted HOV trips. Among QuickRide participants, the number of 3+ carpool trips in the evening increased by 6.1 percent. This suggests that QuickRide may have had some effect in encouraging overall carpooling in the evening peak.

\section{Reasons for Using the HOT Lanes}

When QuickRide enrollees were asked the different reasons for joining the program, among Katy AM participants, 14.5 percent joined the program because they found it too dangerous and stressful to drive on the main lanes. Only 1.1 percent of US 290 AM participants cited the danger and / or stress of driving on the main lanes as their major reason for joining QuickRide. These findings are also supported by the survey findings of Katy and Northwest freeway commuters in November 2003 in which the GPL users expressed their concern to restrict the trucks on freeways.

\section{Usage of the HOT Lanes}

Table 25 compares the volumes on the US290 HOV lane during the morning peak (6:45-8:00) for three periods: June 1999 (prior to 3+ requirement), June 2000 (3+ requirement but prior to QuickRide), and June 2001 (3+ requirement and QuickRide). The change from HOV2+ to HOV3+ in June 2000 caused the volume of HOV2s to drop 62.4 percent during the morning 
peak while 3-person vehicles increased by 60.7 percent. However, the total volume on the HOV lane decreased by 44.5 percent in the morning peak. The addition of the QuickRide program caused the HOV2 volume to increase 40.3 percent between 2000 and 2001, while the HOV3 volume changed relatively little ( -2.7 percent). Additionally, the total volume of the HOV lane increased 21.1 percent.

Table 25 Total US 290 HOV/HOT Lane Volume for Morning Peak (6:45-8:00)

\begin{tabular}{|c|c|c|c|c|c|c|}
\hline \multirow{2}{*}{ Period } & \multicolumn{2}{|c|}{ HOV 2 Vehicles } & \multicolumn{2}{l|}{ HOV 3+ Vehicles } & \multicolumn{2}{c|}{ Total Vehicles } \\
\cline { 2 - 7 } & Vehicles & Change & Vehicles & Change & Vehicles & Change \\
\hline June 1999 & 1268 & & 140 & & 1563 & \\
\hline June 2000 & 477 & $-62.4 \%$ & 225 & $60.7 \%$ & 867 & $-44.5 \%$ \\
\hline June 2001 & 669 & $40.3 \%$ & 219 & $-2.7 \%$ & 1050 & $21.1 \%$ \\
\hline
\end{tabular}

For 2003, there was an average of 86.4 QuickRide users during the morning period on Katy Freeway, 54.9 during the afternoon period on Katy Freeway, and 66.8 during the morning period on the Northwest Freeway. This total of 208.1 QuickRide trips per day is relatively small, but with limited capacity on the single HOV lanes total usage must remain limited (Burris and Stockton, 2004). By September 2006, there were 2000 QuickRide accounts. Because of the daily participation rate of 10 percent, there was only an average of 200 QuickRide trips per day. So, HOT lanes in Huston were underutilized (Smith, 2007).

The objectives of the QuickRide program were to increase person-throughput in the Katy Freeway corridor during peak periods; and increase travel speeds on the GPLs during the peak periods, assuming that many vehicles currently using the GPLs will divert to the HOV lane.

By allowing the additional HOV2s during the peak period, the person throughput of the HOT lanes increased however, the QuickRide usage was too small to increase the person throughput of the corridor. Also, no change in the travel speed of the GPLs can be expected because of the 
few travelers shifting to the HOT lanes during the peak period. Therefore, in terms of objectives the QuickRide program cannot be termed as a success. 


\section{CHAPTER VII}

\section{I-15 EXPRESS LANES, SALT LAKE CITY}

Similar to Houston HOT lanes, I-15 Express Lanes are also different in terms of HOT lanes operations from other existing HOT lanes. The Express Lanes have monthly pass for the SOVs. Therefore, it is discussed in a separate chapter and has not been compared to other HOT lanes.

\section{Introduction}

The I-15 Express Lanes started in September 2006 and extend from 600 North in Salt Lake City to University Parkway (SR-265) in Orem (see Figure 21). The Express Lanes are 38 miles long and separated from the GPLs using the double-white lines. The Express Lanes have one lane in each direction and 18 entrance and exit points along the corridor. HOV2+, vanpools, buses, motorcycles, Alternative (Clean) Fuel Vehicles, and emergency vehicles can use the lanes for free. SOVs are allowed with a monthly permit for $\$ 50$ and decals which are attached to their windshields and rear glass. From September 2006 till May 2007, subscriptions were limited to 1,350 solo drivers per month. In May 2007, the number of subscriptions was increased to 2,200. In fall 2008 UDOT added six miles to the system from State Street in Farmington to 200 North in Kaysville. 


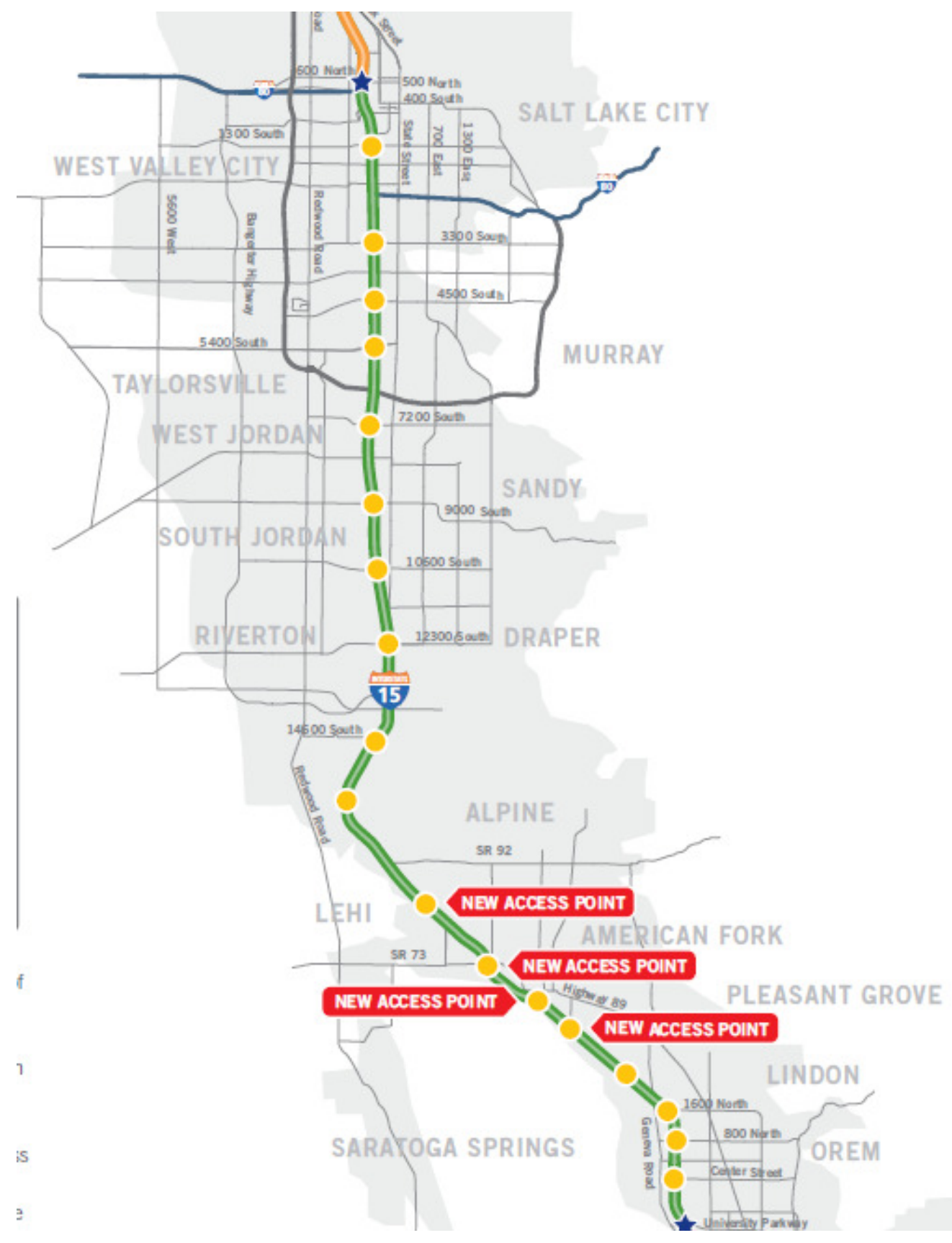

Figure 21 Location of I-15 Utah Express Lanes 38 miles section from University Parkway in Orem to 500 North in Salt Lake City (Utah DOT website)

\section{Previous HOV Operations}

I-15 is a major freeway oriented in the north-south direction in the Salt Lake Valley. In May 2001, 16 miles of HOV lanes were opened on I-15. HOV lanes in the Salt Lake Valley operated between 600 North and 10600 South. The HOV lanes were one lane in each direction and had a 
painted separation from the GPLs. The HOV lanes were operated 24 hours a day and 7 days a week and buses, motorcycles, vanpools, 2+ carpools, Alternative Fuel Vehicles and emergency vehicles were eligible to use them. Vehicles weighing over 12,000 lbs and vehicles towing trailers were not allowed in the HOV lanes even if they satisfied the minimum occupancy requirement. There was HOV-only on-ramp and off-ramp at 400 South (downtown Salt Lake City) to facilitate direct HOV lane entry and exit (Martin et al., 2007).

\section{Why Were HOT Lanes Considered?}

The University of Utah Traffic Laboratory (UTL) did an evaluation project on HOV lanes from 2000 to 2005 for the UDOT and found that HOV lanes carried the minimum number of vehicles required for justification of the HOV existence in peak periods, thus leaving unused capacity on the HOV lanes. Since there was a perception that the HOV lanes were underutilized, Utah DOT decided to conduct an experiment by permitting a limited number of single drivers to pay to use that available capacity (Martin et al., 2007).

\section{Objectives of HOT Lanes}

The objectives of the HOT lanes were to:

- relieve traffic in the GPLs by diverting fee paying SOVs to the carpool lane. Consequently, the travel times for drivers in the GPLs should be decreased without negatively impacting the travel times in the Express Lanes and the value of carpooling (Martin et al., 2007).

- maintain $55 \mathrm{mph}$ for 90 percent of the peak periods on weekdays by limiting the number of permits purchased by SOVs

- clearly define toll rates to the driver.

\section{Changes in the Corridor Due to HOT Lanes}

Previously HOV lanes could be accessed from anywhere along the corridor. To increase safety and improve enforcement in the Express Lanes, the entire corridor was restriped with 18 exit and entrance points. The access points are marked by a white dotted line, while the rest of the corridor is marked with double-white solid lines. Each entry/exit location point is 3,000 feet in 
length, providing motorists with more than 0.5 mile to enter and exit the lanes. Two eight-inch white lines separated by eight inches of space offer a two-foot buffer zone between the Express Lanes and the GPLs. It is illegal to cross a double-white line and, therefore, permissive movements in and out of the carpool lanes are not allowed, except in the specific locations that have white dotted striping, rather than the two solid lines (Martin et al., 2007).

\section{Enforcement Efforts}

Utah Highway Patrol Troopers visually enforce the use of the lanes by SOVs by watching for the decals in the front and back windows of vehicles. Two additional state patrol vehicles have been purchased to help with enforcement (Martin et al., 2007).

\section{Perceptions of Access Points and Safety of the Express Lanes}

Surveys were conducted in May and July 2007 in which the respondents consisted of a random sample of I-15 users. The method adopted was face-to-face personal interviews allowing researchers directly interacting with the respondents. In total, 200 surveys were collected. Only 3 respondents were Express Lanes subscribers (paying SOVs). There is some evidence from the survey which showed the increased inconvenience of access points for the Express lane users all the time.

When respondents were asked if they always use the Express lane when not driving alone, less than a quarter (22 percent) respondents answered positively. Among those who answered negatively, 44 percent mentioned problem in changing lanes (which can be connected to the dissatisfaction with entrance/exit points) as one of the reasons for not using the Express Lanes (Martin et al., 2007).

Inconvenience of access points was also revealed when respondents were asked to state what they do not like about the Express Lanes. A majority (51 percent) of those who gave comments had negative opinion about access to the lane (e.g. frequency, safety, or length of entrance/exit points, or their existence at all) (Martin et al., 2007). 
When respondents were asked to rate their impression of the efficiency of the Express Lanesrespondents rated the exit/entrance points as the lowest (2.78 out of 5) "which was also expected since I-15 users were accustomed to unlimited (unrestricted) access while HOV lanes were operating”. However, the safety of Express Lanes was rated relatively high (4 out of 5). Moreover, 57 percent respondents think that the Express Lanes have improved safety on the I-15 corridor (Martin et al., 2007).

More than 50 percent of the respondents agreed to "drivers jump in and out of the Express Lanes dangerously" and "Express lane violations are common during peak hours." Double white line violations were also reflected when respondents were asked to identify common Express Lane violations. 79 percent of respondents stated that crossing the double-white line is the most common violation. However, to the surprise of the researchers, according to the Highway Patrol, it is not a violation to cross a double-white line to let someone pass you (Martin et al., 2007).

In many ways, the above findings indicate that the change of access points from unrestricted (in previous HOV lanes) to limited access points lead to some inconvenience for the users. However, the majority of the Express lane users think that the Express Lanes have improved the safety and safety was also rated quiet high. These findings indicate that even though limited access points lead to the increased inconvenience of Express lane users to access the Express Lanes, it lead to the increased safety because of more predictable entry points.

\section{Impact on the Corridor}

The following are the different measures of effectiveness (MOEs) examined by the research for the period March to August, 2007(Martin et al., 2007):

\section{Person Throughput}

On an average, in the AM peak period, the Express lane moved 8 percent more people than an average GPL, in 46 percent fewer vehicles. While Express Lanes are more effective in the PM peak period when on an average Express Lanes move 58 percent more people than an average GP Lane, in 24 percent fewer vehicles (Martin et al., 2007). 


\section{Modal Split}

The majority of peak period traffic (70-80 percent) in the Express Lanes was HOVs (see Table 26). Also, the proportion of SOVs in the PM peak is extremely small. However, the most surprising fact is that the percentage of violators is very close to the percentage of subscribers (Martin et al., 2007).

Table 26 Mode Split in the Express Lanes in the AM and PM Peak Hours (March through August 2007)

\begin{tabular}{|l|c|c|}
\hline & AM Peak Hour & PM Peak Hour \\
\hline HOV2+ & $70.04 \%$ & $77.59 \%$ \\
\hline SOVs & $13.79 \%$ & $2.86 \%$ \\
\hline Clean Fuel Vehicles & $2.86 \%$ & $1.34 \%$ \\
\hline Violators & $9.32 \%$ & $7.87 \%$ \\
\hline Motorcycles & $3.10 \%$ & $2.57 \%$ \\
\hline Buses & $0.66 \%$ & $1.05 \%$ \\
\hline Other & $0.22 \%$ & $1.33 \%$ \\
\hline
\end{tabular}

As observed that the proportion of SOVs during the AM peak hour is greater than in the PM peak hour. Since the Express Lanes have monthly pass for SOV users, why the travelers do not use it both ways (AM and PM) on a similar basis is not clear.

\section{Travel Time}

Table 27 shows the peak period travel times in the GPLs and the Express Lanes. (The travel times are reported from 400 North to 800 South in Orem-points close enough to the end points of the Express Lanes). The travel time was collected suing the travel time runs using the GPS. The travel time savings are higher in the PM peak but the travel time reliability of the Express Lanes is better during the AM peak than the PM peak.

Table 27 Travel Time Summary for May, June, July and August 2007

\begin{tabular}{|l|c|c|c|c|c|c|}
\hline & \multicolumn{3}{|c|}{ AM Peak Period } & \multicolumn{3}{c|}{ PM Peak Period } \\
\hline & GPLs & Express Lanes & Difference & GPLs & Express Lanes & Difference \\
\hline Average (minutes) & 40.96 & 33.78 & 7.17 & 49.69 & 39.41 & 9.25 \\
\hline $\begin{array}{l}\text { Standard } \\
\text { Deviation }\end{array}$ & 4.1 & 1.26 & 3.31 & 8.09 & 6.11 & 4.15 \\
\hline
\end{tabular}




\section{Speed}

Table 28 shows the percentage of times GPLs and Express Lanes were congested and average and standard deviation of speeds. During both in the AM and PM periods the average speed on the Express Lanes is significantly higher (at 95 percent confidence level) than the average speed on the GPLs. Also, the standard deviation values show that the reliability of speed in Express Lanes is superior to the GPLs but decreases considerable in the PM period.

Looking at the average speed of GPLs in the PM peak (around $10 \mathrm{mph}$ slower than AM) and the above observation (see Table 26) of increased carpooling in PM (78 percent in PM and 70 percent in AM) indicate that more congestion in GPLs during PM peak might have encouraged more travelers to carpool in Express Lanes.

Table 28 Percent of Congested Travel, Average and Standard Deviation of Speeds

\begin{tabular}{|l|c|c|c|c|}
\hline & \multicolumn{2}{|c|}{ AM Peak Hour } & \multicolumn{2}{c|}{ PM Peak Hour } \\
\hline SPEED MEASURE & GPL & Express Lanes & GPL & Express Lanes \\
\hline Speeds less than $\mathbf{4 5} \mathbf{~ m p h}$ & $22.89 \%$ & $2.97 \%$ & $39.71 \%$ & $14.46 \%$ \\
\hline Average Speed (mph) & 58.74 & 69.95 & 49.58 & 61.09 \\
\hline Standard Deviation (mph) & 19.95 & 8.99 & 23.02 & 14.29 \\
\hline
\end{tabular}

The efficiency of Express Lanes is indicated by the fact that after the conversion of HOV lanes (which were underutilized) to HOT lanes in 2006 the speed of both the Express Lanes and the GPLs increased by around $3 \mathrm{mph}$ in both lanes. Note that the speeds in both the lanes were decreased in the year before the Express Lanes opened (from 2004 to 2005). Possible explanations for this were given as follows (Martin et al., 2007):

1) Restricted entrance/exit points do not allow speed disruptions

2) Paying subscribers expect to benefit and are more likely to travel faster, while in the Express lane

3) In 2005, there were no obvious advantages of using the HOV lane in the AM peak, and so HOV users matched GP speeds (with little incentive to travel faster) — in 2007 when the GP Lanes are congested, HOV (now Express lane) users actively opt to leave the GPLs and seek higher speeds. 


\section{CHAPTER VIII}

\section{CONCLUSIONS AND RECOMMENDATIONS}

To begin, many of the most interesting findings from each HOT lane are examined.

\section{SR 91 Express Lanes and I-95 Express Lanes}

1) Transit ridership on SR 91 remained almost stable while ridership increased by 30 percent on I-95 one year after the Express Lanes opened. One key difference between two Express Lanes is that the express bus on the SR 91 corridor does not travel on the Express Lanes while express bus on I-95 does. With express lanes on I-95, the travel time of buses decreased by 17 minutes. Therefore, an improvement in HOT lane performance was able to attract added transit trips in I-95.

2) The two Express Lanes have a similar separation mechanism (flexible poles) from the GPLs and the Express Lanes have no side shoulder. The I-95 Express Lanes were narrower, only 11 feet wide. The safety perceptions of the Express lane users in the two corridors differed. There were no negative comments from SR 91 survey respondents regarding safety issues of these Express Lanes and 40 percent of the respondents mentioned safety as one of the reasons for using the Express Lanes. 22 percent of I-95 survey respondents mentioned the Express Lanes were less safe than the previous HOV lanes. However, 18 percent of toll paying and carpool users of Express Lanes also mentioned (see Table 9) safety perceptions in the lanes as one of the reasons for using the Express Lanes. FDOT did not find any safety concerns in the day-to-day monitoring of crashes. One of the reasons for this difference between the perceived safety of the travelers in the two corridors might be attributed to narrower lanes in I-95 Express Lanes. Another possible explanation could be the difference in the traffic volumes of the two corridors. At the time of Express Lanes implementation, ADT of SR 91 corridor was around 200,000 vpd while it was around 290,000 vpd in I-95. This means that the feasibility of a separation mechanism might be related to corridor specific conditions and its success in one corridor could not be generalized. 
Previously researchers have examined the safety of a roadway in relation to its lane width. Zegeer et al, (1987), Goldstine (1991), DeLuca (1985), Hadi et al. (1995) and Choueiri et al. (1994) showed an inverse relationship between lane width and crash occurrences. That is, an increasing lane width will result in fewer crashes. However, contradictory results were also reported by Potts et al., 2007 who found no general indication that the use of lanes narrower than $12 \mathrm{ft}$ on urban or suburban roads would result in increased crash frequencies. Dart and Mann, 1970 also found insignificant differences in the crash rates of roads with lane widths between 11 and $12 \mathrm{ft}$. Their findings were also supported by the National Cooperative Research Program (NCHRP) Report 197 which concluded that there was a decrease in the accident rate as the pavement or lane width increased up to $11 \mathrm{ft}$ but accident rate remained fairly constant above $11 \mathrm{ft}$.

It should be noted that none of the above research was done in the context of managed lanes (which leads to increased merging) and/or with a separation mechanism like flexible poles. There is a little experience of this separation mechanism and narrower lane width both in practice as well as in research. Therefore, Express lane authorities should closely monitor this aspect of safety to ensure the safe operations of the Express Lanes with this design exception.

3) After both Express Lanes started, there was a 40 percent increase in HOV3+ vehicles during the PM peak hours in the SR 91 corridor while HOV3+ vehicles increased by 10 percent in the I-95 corridor (but decreased by 64 percent in the Express Lanes). Among the potential reasons for the different impact on HOV3+ in the two corridors were: (i) no previous HOV lane incentive for HOV3+ on SR 91, (ii) induced traffic in the SR 91 corridor with some of this being HOV3+ traffic, (iii) strict HOV3+ carpool registration requirements and access inconvenience with the I-95 express lanes (v) only one-way operation of I-95 express lanes (until January 2010) and (vi) availability of an express bus on I-95 Express Lanes. Therefore, same policy of carpool eligibility has different impacts on the two corridors because of many endogenous (like bus availability and registration requirement) as well as exogenous factors (like induced traffic). This finding underscores 
the importance of considering all the different factors before comparing the impacts of HOT lanes in two different corridors.

4) On the SR 91 corridor, Express Lanes did little to encourage or discourage HOV2 carpools. On I-95, HOV2 volume decreased by 38 percent in the Express Lanes and by 15 percent in the GPLs. Overall, as compared to a conventional HOV lane (Sullivan, 1998) with 60 percent of HOV2 in the corridor using the HOV lanes, SR 91 Express Lanes were used by 30 percent and I-95 Express Lanes by 23 percent HOV2s in the 2 hour PM peak. Therefore, HOT lane projects with priced HOV2 access lead to reduced usage of HOT lanes by the HOV2 as compared to a scenario with free HOV2 access.

5) Induced traffic on the SR 91 corridor after the Express Lanes opened has significantly affected several aspects of the corridor. Due to induced traffic (i) the delay of the corridor became worse with time, (ii) there was a significant increase in HOV3+ vehicles during the PM peak and (iii) there was a significant increase in the number of SOVs in the corridor ( 80 percent of the induced traffic was SOVs) which effectively lead to the reduced average vehicle occupancy of the corridor even with the increase in HOV3+ vehicles. Therefore, SR 91 shows how external factors, like induced traffic, play an important role in deciding the benefits achieved from a HOT lane project.

\section{I-15, San Diego and I-25, Denver Express Lanes}

6) Over a period of three years since the HOT lanes started in I-15(December 1996 November 1999) weekday carpool usage of I-15 Express Lanes increased by more than 40 percent. During the first three years of I-25 Express Lanes operations (June 2006 July 2009) weekday carpool usage remained at the pre-Express lane rate. Conversely, toll usage reached similar levels in both the Express Lanes.

7) Both of the Express Lanes are barrier separated from the GPLs with no intermediate access points. More than 75 percent of survey respondents for both the Express Lanes were satisfied with the safety aspect of their respective Express Lanes. However, in the I25 Express Lanes survey, users were most dissatisfied with inconvenience of access points (among all the Express lane aspects). On I-15, researchers partially attributed the decreasing number of carpools on I-15 GPLs from 1996 through 1999 due to access inconvenience. Therefore, there is a trade-off between the safety and the access 
convenience of the Express Lanes. With concrete barrier the safety perceived by the Express lane users increased however it came at the cost of increased inconvenience. Also, access inconvenience may also be the reason why carpools did not increase in the I25 Express Lanes in addition to the lower congestion level as noted in the previous point.

8) Both of the Express Lanes had little impact on transit ridership in the corridor. The Inland Breeze bus route using the I-15 Express Lanes in the peak period (and using the GPLs during non-peak hours) served mostly (75 percent) reverse commuters and the bus ridership did not reach the goal of 750 daily riders after two years. After the Express Lanes implementation in I-25 there was almost no change in bus ridership. However, there were some indications that the implementation on I-25 Express Lanes might have offset the effect of reduced gas prices (normally leading to lesser transit use) . Therefore, the lack of impact on transit due to Express lane implementation in the two corridors was affected by traveler characteristics on I-15 and an external factor (gas prices) on I-25.

9) There were indications of a potential relationship between gas prices, carpools and toll usage. However, the dependence of carpools and toll paying SOVs on gas prices varied (see Figure 10). On one hand, carpool usage increased and then decreased along with gas prices. However, toll usage generally decreased during the period when gas prices increased and then decreased. As carpools and toll paying SOVs decreased, transit ridership increased during that period. Therefore, these potential mode shift indications (from Express Lanes to transit) due to change in gas prices underscore the importance of exogenous factors like gas prices in deciding the usage of Express Lanes. However, future research is required to better understand this mode shift behavior due to gas price fluctuation using the traveler surveys.

10) A steady decreasing trend of toll users (irrespective of gas price changes) on I-25 Express Lanes (see Figure 10) raised some concern regarding the pricing policy of the Express Lanes with a fixed peak hour toll of $\$ 3.25$. There is a possibility that some toll users would have initially made a decision to use the HOT lanes but over time decided the toll is too high for the time savings. In contrast to this, in a dynamic pricing HOT lane, the toll is set based on travel speeds and may be more enticing in situations where all lanes are flowing well (GPL and HOT) and therefore the HOT lanes would have a low toll. These arguments underscore the need for future research on HOT lanes to compare the 
effectiveness of two pricing mechanisms in balancing the demand of two user typestolled and HOVs (and transit) and maintaining the free flow of HOT lanes at the same time.

\section{I-394 HOT Lanes (Diamond Lane Section) and SR 167 HOT Lanes}

11) Both the HOT lanes have similar separation mechanisms (double white line) and limited access points. In fact, SR 167 HOT lanes based their design on the MnPASS design. Additionally, both the HOT lanes used toll revenue to fund increased enforcement for the HOT lane operations. However, there is a significant difference between the perceptions of safety by the travelers on the two HOT lanes. In an I-394 panel survey, more than 75 percent of respondents were satisfied with the ease of identifying the access points and with the safety of merging into the HOT lanes. On the other hand, participants in the focus group conducted for SR 167 HOT lanes were concerned regarding the safety of getting in and out of the HOT lanes and a majority of them mentioned problems with double-white lines including drivers illegally crossing the lines and limited access to enter and exit the lanes. It should be noted that buffer separation is twice as wide in SR 167 as it is in I-394 HOT lanes. However, GPL traffic is more congested on SR 167. The success of the separation mechanism on I-394 indicates that with continued enforcement and travelers getting used to the modified design, safety perceptions of the travelers might improve with time. Conversely, the difference between the perceived safety of the two HOT lanes might also be due to the different traffic conditions in the two corridors and may not change with time. This would be a useful aspect of the lane to recheck in 2010.

12) There was a strong positive correlation between the weekday carpool usage and gas prices on the SR 167 HOT lanes. Carpooling clearly followed the trend of gas prices (see Figure 19). With decreasing gas prices, carpool usage decreased, toll usage increased and bus ridership decreased. These findings indicate that with reduced gas prices, carpoolers and transit riders shifted to SOV mode (both in HOT and the GPLs) and auto mode respectively. It should be noted that in both the cases SR 167 and I-25 (as discussed above), the variation of carpool usage with gas prices significantly differed from that of 
toll usage. It shows that the elasticity of the two modes is different with respect to the gas prices.

These findings highlight the effects gas price fluctuations have on the mode choice of travelers in a HOT lane scenario. Therefore, the usage of HOT lanes, to some extent, is dependent on exogenous factors like gas price which is generally not included as one of the elements in the planning of HOT lanes. It also implies that the performance of HOT lanes should be judged in light of the gas prices in the respective locations and it becomes even more crucial when the two HOT lanes are compared based on their usage.

\section{I-10 and US 290 HOT Lanes in Houston}

13) Houston HOT lanes are the only HOT lanes which do not allow access to SOVs. This policy was based on high demand of the single lane corridors which could have overcrowded the HOT lanes even with pricing.

14) There were evidences which showed that Houston HOT lanes had a little impact on the transit ridership. A survey of non-QuickRide commuters found that some transit riders were not satisfied with the service and operations of METRO buses. And, a survey of QuickRide commuters found very few trips (15) shifted to HOT lanes from transit.

15) A QuickRide user survey found that 50 percent of the QuickRide trips (paying HOV2s) were diverted from SOVs on the GPLs.

16) The presence of a high proportion of trucks on the GPLs and barrier separated HOT lanes seemed to increase the safety perception of the HOT lane users (since HOT lanes do not allow the trucks) and some users also mentioned safety as one of the reasons for using the HOT lanes.

17) Houston HOT lanes did not make any significant impact on the GPLs because of peak hour only operations and HOV2 pricing. By 2007, there were on average 200 QuickRide trips per day. However, it was successful in increasing the person throughput in the HOT Lanes during the peak period.

\section{I-15 Express Lanes in Salt Lake City}

18) With Express Lanes implementation, the unrestricted access to the previous HOV lanes was greatly reduced. As a result of this change, the Express lane users mentioned 
increased inconvenience of accessing the lanes and rated access points efficiency as the lowest. On the other hand, a majority of the respondents perceived improved safety with Express Lanes. These findings indicate the trade-off between limited access and increased safety of the Express Lanes.

Also, reduced speed disruptions due to limited access points was found by researchers as one of the reasons for increased efficiency of the corridor after the Express Lanes opened The speed in the Express Lanes as well as the GPLs increased by $3 \mathrm{mph}$.

19) There were indications of increased carpooling in the Express Lanes due to increased congestion in the GPLs. In the PM peak period carpooling in the Express Lanes was 7 percent higher than in AM peak (and SOVs lesser by 11 percent). Express Lanes Also, the GPLs in the PM peak were, on average, $10 \mathrm{mph}$ slower than AM peak. Therefore, these findings again show some relation between GPL congestion and the carpooling.

\section{Safety Implications of the HOT Lanes}

The presence of different kinds of separation mechanisms on the different HOT lanes around the country provides an opportunity to compare the perceived safety of the lanes. Table 29 summarizes the different separation mechanisms used by the HOT lanes and the safety perceptions of the travelers. 
Table 29 Separation Mechanism and Safety Perception of the HOT Lane Users

\begin{tabular}{|c|c|c|c|}
\hline HOT Lane Facility & $\begin{array}{l}\text { HOV Lane Separation } \\
\text { Mechanism and Access } \\
\text { Points }\end{array}$ & $\begin{array}{l}\text { HOT Lane Separation } \\
\text { Mechanism and Access } \\
\text { Points }\end{array}$ & Perception of Travelers \\
\hline $\begin{array}{l}\text { I-394 HOT lanes- } \\
\text { Diamond Section, } \\
\text { Minneapolis }\end{array}$ & $\begin{array}{l}\text { Double-White lines, } \\
\text { Unrestricted access }\end{array}$ & $\begin{array}{l}\text { Double-White lines }(2 \mathrm{ft} . \\
\text { buffer }), \text { Limited access points } \\
\text { ( } 6 \text { in each direction) }\end{array}$ & $\begin{array}{l}\text { Most respondents (66 percent) were satisfied with the safety of } \\
\text { merging into the HOT lanes. } 87 \text { percent of surveyed HOT lane users } \\
\text { indicated that there is no problem merging in to the MnPASS lanes } \\
\text { from the GPLs. }\end{array}$ \\
\hline $\begin{array}{l}\text { SR } 167 \text { HOT lanes, } \\
\text { Seattle }\end{array}$ & $\begin{array}{l}\text { Double-White lines, } \\
\text { Unrestricted access }\end{array}$ & $\begin{array}{l}\text { Double-White lines (4 ft. } \\
\text { buffer), Limited access points } \\
\text { (6 NB and } 4 \mathrm{SB} \text { ) }\end{array}$ & $\begin{array}{l}\text { Members of a Focus group expressed concern regarding getting cut } \\
\text { off as they exit the HOT lanes. Complaints also included insufficient } \\
\text { time to exit the freeway after crossing the dotted white lines, not } \\
\text { enough space to enter/exit at the dotted white lines, and not enough } \\
\text { places where the dotted white lines can be crossed. }\end{array}$ \\
\hline $\begin{array}{l}\text { I-15 Express Lanes, } \\
\text { Salt Lake City }\end{array}$ & $\begin{array}{l}\text { Double-White lines, } \\
\text { Unrestricted access }\end{array}$ & $\begin{array}{l}\text { Double-White lines ( } 2 \mathrm{ft} . \\
\text { buffer), Limited access points } \\
(17)\end{array}$ & $\begin{array}{l}44 \text { percent of those who did not use the Express Lanes, even while } \\
\text { carpooling mentioned inconvenient access as one of the reasons. A } \\
\text { majority of the respondents mentioned access as one of the aspects of } \\
\text { the Express Lanes they did not like. However, the majority of the } \\
\text { respondents also perceived improved safety with the Express Lanes. }\end{array}$ \\
\hline
\end{tabular}


Table 29 Continued

\begin{tabular}{|c|c|c|c|}
\hline HOT Lane Facility & $\begin{array}{l}\text { HOV Lane Separation } \\
\text { Mechanism and Access } \\
\text { Points }\end{array}$ & $\begin{array}{l}\text { HOT Lane Separation } \\
\text { Mechanism and Access } \\
\text { Points }\end{array}$ & Perception of Travelers \\
\hline $\begin{array}{l}\text { I-95 Express Lanes, } \\
\text { Miami }\end{array}$ & $\begin{array}{l}\text { Painted Stripes, } \\
\text { Unrestricted access }\end{array}$ & $\begin{array}{l}\text { Flexible pylons, no } \\
\text { intermediate access points, } \\
\text { narrower Express Lanes ( } 11 \\
\text { ft.), no side shoulder for } \\
\text { Express lanes }\end{array}$ & $\begin{array}{l}\text { Express Lane users' comments indicate serious concerns regarding } \\
\text { vehicles crossing over the poles and narrower lanes with no shoulders. } \\
22 \text { percent of survey respondents mentioned Express Lanes have } \\
\text { become unsafe. According to FDOT - no serious safety concerns } \\
\text { were found during the monitoring of the lanes. }\end{array}$ \\
\hline $\begin{array}{l}\text { SR } 91 \text { Express Lanes, } \\
\text { Los Angeles }\end{array}$ & No HOV lanes previously & $\begin{array}{l}\text { Flexible Poles with no side } \\
\text { shoulder for the Express } \\
\text { Lanes, no intermediate access } \\
\text { points }\end{array}$ & $\begin{array}{l}\text { When asked for reasons other than travel time savings to use the } \\
\text { Express lanes, } 40 \text { percent of the survey respondents mentioned } \\
\text { driving comfort and safety in the Express Lanes. No comments } \\
\text { regarding the access points were mentioned by the survey } \\
\text { respondents. }\end{array}$ \\
\hline
\end{tabular}


Table 29 Continued

\begin{tabular}{|l|l|l|l|}
\hline HOT Lane Facility & $\begin{array}{l}\text { HOV Lane Separation } \\
\text { Mechanism and Access } \\
\text { Points }\end{array}$ & $\begin{array}{l}\text { HOT Lane Separation } \\
\text { Mechanism and Access } \\
\text { Points }\end{array}$ & Perception of Travelers \\
\hline $\begin{array}{l}\text { I-10 and US 290 HOT } \\
\text { lanes, Houston }\end{array}$ & $\begin{array}{l}\text { Barrier Separated, } \\
\text { Intermediate access points } \\
(2 \text { on I-10 and 4 on US } \\
290)\end{array}$ & Same as HOV & $\begin{array}{l}\text { Safety perceptions in the HOT lanes were mentioned by the I-10 HOT } \\
\text { lane users as one of the reasons for using the HOT lanes. }\end{array}$ \\
\hline $\begin{array}{l}\text { I-25 Express Lanes, } \\
\text { Denver }\end{array}$ & $\begin{array}{l}\text { Barrier Separation, No } \\
\text { intermediate access points }\end{array}$ & Same as HOV & $\begin{array}{l}89 \text { percent of survey respondents were satisfied with the safety aspect } \\
\text { of the Express Lanes (for example reduced chances of being in an } \\
\text { accident) and 60 percent among them very satisfied. However, the } \\
\text { biggest dissatisfaction among I-25 Express lane users came with } \\
\text { convenience of access points with almost one-third (32 percent) } \\
\text { dissatisfied with this aspect }\end{array}$ \\
\hline
\end{tabular}


Table 30 HOT Lane Facilities, Their Objectives, Policy Changes and Impacts

\begin{tabular}{|c|c|c|c|}
\hline $\begin{array}{l}\text { HOT Lane Facility } \\
\text { (Start date) }\end{array}$ & $\begin{array}{l}\text { Objectives of HOT Lane } \\
\text { Conversion }\end{array}$ & $\begin{array}{c}\text { Important Policies/Changes Adopted } \\
\text { During the Conversion }\end{array}$ & Impacts \\
\hline $\begin{array}{l}\text { SR } 91 \text { Express Lanes, } \\
\text { Los Angeles } \\
\text { (December 1995) }\end{array}$ & $\begin{array}{l}\text { - For-profit built and operated by } \\
\text { private company }\end{array}$ & $\begin{array}{l}\text { - Previously no HOV lanes on the } \\
\text { corridor } \\
\text { — Carpool eligibility for free access } \\
\text { was kept as HOV3+ (until January } \\
\text { 1998) } \\
\text { - The Express Lanes are separated } \\
\text { from the GPLs using flexible poles } \\
\text { - No intermediate access to the } \\
\text { Express Lanes }\end{array}$ & $\begin{array}{l}\text { - Increase in ADT in the first year in corridor matched } \\
\text { closely with the ADT increase of the Express Lanes } \\
\text { - } 60 \text { percent of the total ADT increase (28,000 vpd) in } \\
\text { the corridor in the first years was due to induced } \\
\text { traffic } \\
\text { - After the Express Lanes opened, freeway delay was } \\
\text { reduced from } 30-40 \text { minutes to } 5 \text { minutes. Due to } \\
\text { induced traffic, delay was more than doubled to } 12- \\
13 \text { minutes by June } 1997 \\
\text { - HOV3+ increased by } 40 \text { percent during the PM peak } \\
\text { period which was attributed mostly to induced traffic. } \\
\text { Induced traffic during the PM peak consisted } 12 \\
\text { percent of HOV3+. } \\
\text { HOV2+ traffic volumes in the corridor remained } \\
\text { relatively stable after the Express Lanes } \\
\text { Bus ridership remained stable. Also buses do not use } \\
\text { the Express Lanes. Indications of rail ridership } \\
\text { decreasing due to the Express Lanes }\end{array}$ \\
\hline
\end{tabular}




\section{Table 30 Continued}

\begin{tabular}{|c|c|c|c|}
\hline $\begin{array}{l}\text { HOT Lane Facility } \\
\text { (Start date) }\end{array}$ & $\begin{array}{l}\text { Objectives of HOT Lane } \\
\text { Conversion }\end{array}$ & $\begin{array}{c}\text { Important Policies/Changes Adopted } \\
\text { During the Conversion }\end{array}$ & Impacts \\
\hline $\begin{array}{l}\text { I-15 Express Lanes, } \\
\text { San Diego } \\
\text { (December 1996) }\end{array}$ & $\begin{array}{l}\text { - Maximize use of the HOV lanes } \\
\text { - Testing ability of SOV access to } \\
\text { HOV lanes to help relieve } \\
\text { congestion on the GPLs } \\
\text { - Funding new transit and HOV } \\
\text { improvements in the corridor } \\
\text { - Using a market-based approach } \\
\text { to set tolls }\end{array}$ & $\begin{array}{l}\text { - SOVs allowed to use the lanes for } \\
\text { a toll decided by dynamic pricing } \\
\text { - Half of the excess revenue used to } \\
\text { fund Inland Breeze Bus route. } \\
\text { - Shoulder pricing was reduced to } \\
\text { encourage FasTrak usage during } \\
\text { non-peak hours. }\end{array}$ & $\begin{array}{l}\text { - Inland Breeze bus route (funded by the project) } \\
\text { served mostly (75\%) reverse commuters going the } \\
\text { opposite direction of Express Lanes operation. In } \\
\text { January 2007, the bus service was discontinued. } \\
\text { - Total weekday usage of Express Lanes increased } \\
\text { from } 7685 \text { (pre-Express Lanes) to 15,000 in three } \\
\text { years. } \\
\text { - Congestion on the GPLs was alleviated by directing } \\
\text { the increasing traffic on corridor to HOT lanes }\end{array}$ \\
\hline $\begin{array}{l}\text { I-10 HOT lanes, } \\
\text { Houston (January } \\
\text { 1998) and US } 290 \\
\text { HOT lanes, Houston } \\
\text { (November 2000) }\end{array}$ & $\begin{array}{l}\text { - Increase person-throughput in the } \\
\text { Katy Freeway corridor during } \\
\text { peak periods. } \\
\text { - Increase travel speeds on the } \\
\text { GPLs during peak periods, } \\
\text { assuming that many vehicles } \\
\text { currently using the GPLs will } \\
\text { divert to the HOV lane. } \\
\text { Efficiently manage demand } \\
\text { without adverse operating } \\
\text { impacts on both the HOT lane } \\
\text { and the GPLs. }\end{array}$ & $\begin{array}{l}\text { HOV2 allowed to access the HOT } \\
\text { lanes during the peak periods for a } \\
\text { flat per trip fee of } \$ 2\end{array}$ & $\begin{array}{l}\text { - A QuickRide user survey found that } 50 \text { percent of the } \\
\text { QuickRide trips (paying HOV2s) were diverted from } \\
\text { SOVs on GPLs. } \\
\text { - Person throughput of the HOT lanes increased as } \\
\text { compared to HOV3+ requirement period } \\
\text { - HOT lanes could not make any significant impact on } \\
\text { GPL congestion because of peak hour only operations } \\
\text { and HOV2 pricing. }\end{array}$ \\
\hline
\end{tabular}


Table 30 Continued

\begin{tabular}{|c|c|c|c|}
\hline $\begin{array}{l}\text { HOT Lane Facility } \\
\text { (Start date) }\end{array}$ & $\begin{array}{l}\text { Objectives of HOT Lane } \\
\text { Conversion }\end{array}$ & $\begin{array}{c}\text { Important Policies/Changes Adopted } \\
\text { During the Conversion }\end{array}$ & Impacts \\
\hline $\begin{array}{l}\text { I-394 Express Lanes, } \\
\text { Minneapolis } \\
\text { (May 2005) }\end{array}$ & $\begin{array}{l}\text { - Improving efficiency of the } \\
\text { corridor by increasing the } \\
\text { number of people and vehicles } \\
\text { using the HOV lane } \\
\text { - Maintaining free flow speed for } \\
\text { transit and carpools in the HOV } \\
\text { lanes } \\
\text { - Using excess revenue, if } \\
\text { available, for transit and highway } \\
\text { improvement in the corridor } \\
\text { - Using toll collection without toll } \\
\text { booths } \\
\text { Employing ITS technology- e.g. } \\
\text { dynamic pricing and in vehicle } \\
\text { electronic enforcement }\end{array}$ & $\begin{array}{l}\text { - SOVs allowed to use the lanes for } \\
\text { a dynamic priced toll } \\
\text { - First HOT lane to adopt striped } \\
\text { separation from the GPLs } \\
\text { - HOVs and transit access changed } \\
\text { from unrestricted to limited access } \\
\text { points on diamond section } \\
\text { - Previous hours of operation } \\
\text { extended- } 1 \text { hour in AM and } 2 \\
\text { hours in PM } \\
\text { Toll based on the location of entry } \\
\text { in to the Express Lanes }\end{array}$ & $\begin{array}{l}\text { - Bus ridership increased after the HOT lanes, } \\
\text { significantly higher than in the control corridor. } \\
\text { - User surveys indicated no negative impact on } \\
\text { carpooling due to the Express Lanes. } \\
\text { - Carpoolers decreased in peak periods. Attributed to } \\
\text { the increase in operational hours } \\
\text { - Project could not generate excess revenue to fund } \\
\text { transit }\end{array}$ \\
\hline $\begin{array}{l}\text { I-25 Express Lanes, } \\
\text { Denver (June 2006) }\end{array}$ & $\begin{array}{l}\text { - Optimizing use of HOV lanes } \\
\text { - Providing a new transportation } \\
\text { option for North I-15 travelers } \\
\text { - Producing enough revenue to } \\
\text { cover all expenses, including } \\
\text { current operational expenses for } \\
\text { the HOV lanes }\end{array}$ & $\begin{array}{l}\text { - Hierarchy of HOT lanes users was } \\
\text { defined with buses at the top } \\
\text { followed by vanpools and 3+ } \\
\text { carpools (second), 2+ carpools } \\
\text { (third), ILEV (fourth) and SOVs } \\
\text { (lowest) } \\
\text { - The minimum peak hour toll in the } \\
\text { Express Lanes was set equal to the } \\
\text { bus fare } \\
\text { Toll triggers based on maintaining } \\
\text { the free-flow speed of buses } \\
\text { The speed of buses is the primary } \\
\text { performance measure }\end{array}$ & $\begin{array}{l}\text { - Express lane users indicated more use of transit due } \\
\text { to the Express Lanes. However, no significant change } \\
\text { in ridership in the first year. } \\
\text { - ADT of carpools in the Express lanes remained very } \\
\text { close to the pre-Express level } \\
\text { ADT of SOVs in the Express Lanes increased } \\
\text { steadily until early } 2008 \text { when it started decreasing. } \\
\text { - Highest weekday usage in first } 3 \text { years in February } \\
2008 \text { resulted in } 1035 \text { vehicles in the peak hour (less } \\
\text { than half of the free-flow capacity) }\end{array}$ \\
\hline
\end{tabular}


Table 30 Continued

\begin{tabular}{|c|c|c|c|}
\hline $\begin{array}{l}\text { HOT Lane Facility } \\
\text { (Start date) }\end{array}$ & $\begin{array}{l}\text { Objectives of HOT Lane } \\
\text { Conversion }\end{array}$ & $\begin{array}{c}\text { Important Policies/Changes Adopted } \\
\text { During the Conversion }\end{array}$ & Impacts \\
\hline $\begin{array}{l}\text { I-95Express Lanes, } \\
\text { Miami } \\
\text { (December 2008) }\end{array}$ & $\begin{array}{l}\text { - Increasing safety, reliability and } \\
\text { throughput of the corridor } \\
\text { - Encouraging rideshare and transit } \\
\text { use }\end{array}$ & $\begin{array}{l}\text { - Carpool eligibility for free access } \\
\text { increased from } 2+\text { to } 3+\text { with strict } \\
\text { definition of carpool and } \\
\text { registration requirement } \\
\text { - Registered Hybrid vehicles } \\
\text { allowed free access } \\
\text { - Corridor restriped to convert one } \\
\text { HOV lane to two HOT lanes per } \\
\text { direction. Narrower (11 ft.) HOT } \\
\text { lanes with no shoulder. } \\
\text { - Striped separation changed to } \\
\text { flexible poles } \\
\text { - Access changed from unrestricted } \\
\text { to no intermediate access points }\end{array}$ & $\begin{array}{l}\text { - Serious safety concerns among the Express lane } \\
\text { users. According to FDOT - no serious safety } \\
\text { concerns were found during the monitoring } \\
\text { - HOV3+ comprises less than a quarter of the toll } \\
\text { exempt vehicles. Majority of the toll exempt trips are } \\
\text { made by Hybrids. } \\
\text { - Some previous carpoolers in HOV lanes broke up in } \\
\text { to toll paying users (SOV/HOV2) or do not use the } \\
\text { Express Lanes } \\
\text { - Travel time of express bus reduced by } 17 \text { minutes and } \\
\text { its ridership increased by } 30 \text { percent comparing same } \\
\text { three months before and after the Express Lanes. } \\
\text { - In the PM peak hour (4PM-5PM), the throughput of } \\
\text { the corridor increased by } 12 \text { percent }\end{array}$ \\
\hline $\begin{array}{l}\text { SR } 167 \text { HOT lanes, } \\
\text { Seattle } \\
\text { (May 2008) }\end{array}$ & $\begin{array}{l}\text { Testing the HOT lane concept's } \\
\text { ability to } \\
\text { maintain the speed and reliability } \\
\text { of the HOV system without } \\
\text { adversely impacting congestion } \\
\text { along the project corridor and/or } \\
\text { the regional highway system } \\
\text { - generate a stream of revenue that } \\
\text { can be used to pay for the } \\
\text { operation and maintenance of the } \\
\text { facility as well as transportation } \\
\text { system improvements. } \\
\text { And, } \\
\text { Assessing the level of public } \\
\text { interest and support for the HOT } \\
\text { lane concept. }\end{array}$ & $\begin{array}{l}\text { - Dynamic pricing was adopted to } \\
\text { ensure that buses and carpools can } \\
\text { keep moving at a good speed } \\
\text { - A share of revenue collected from } \\
\text { HOT lanes dedicated to improve } \\
\text { transit, vanpool, ride-share and trip } \\
\text { reduction services in the corridor } \\
\text { - Corridor restriped to provide } 4 \\
\text { feet, double white line, buffer } \\
\text { between GPLs and HOT lanes } \\
\text { - Access to HOV lanes changed } \\
\text { from unrestricted to limited access } \\
\text { points on HOT lanes } \\
\text { - Buses using the HOV lanes were } \\
\text { fine-tuned to make better use of } \\
\text { HOT lanes }\end{array}$ & $\begin{array}{l}\text { - Bus ridership increased in the first year (2008) and } \\
\text { then decreased in the second year (2008) of HOT lane } \\
\text { operation. This fluctuation was attributed to gas price. } \\
\text { - When not using the toll option on HOT lanes, almost } \\
\text { half of the HOT lane users drive alone on GPLs and } \\
\text { only one-tenth carpool- indicating minimal negative } \\
\text { impact on carpooling } \\
\text { - Carpool usage decreased by more than } 25 \text { percent in } \\
\text { one year and toll usage increased almost three times. } \\
\text { - At its peak usage level of } 9500 \text { users per direction in } \\
\text { July } 2008 \text {, the AM and PM peak hour flow was less } \\
\text { than } 1000 \text { vehicles. } \\
\text { According to the Focus group there are serious safety } \\
\text { concerns with double-white line separation }\end{array}$ \\
\hline
\end{tabular}


Table 30 Continued

\begin{tabular}{|l|l|l|l|}
\hline $\begin{array}{c}\text { HOT Lane Facility } \\
\text { (Start date) }\end{array}$ & \multicolumn{1}{c|}{$\begin{array}{c}\text { Objectives of HOT Lane } \\
\text { Conversion }\end{array}$} & $\begin{array}{c}\text { Important Policies/Changes Adopted } \\
\text { During the Conversion }\end{array}$ & \\
\hline & $-\begin{array}{l}\text { Collecting performance data to } \\
\text { help determine if HOT lanes } \\
\text { could be used effectively in other } \\
\text { locations and what modifications } \\
\text { would be necessary to help } \\
\text { ensure their successful }\end{array}$ & & \\
& implementation. & & \\
& Assessing the socio-economic & & \\
impacts of the facility & & \\
\hline
\end{tabular}




\section{Impacts of the Different HOT Lanes}

Table 30 summarizes the objectives of different HOT lanes, changes made in the corridor due to HOT lanes and the different impacts on their respective corridors. The impacts reported in this table are in the context of the objectives of the HOT lanes.

This research examined:

- the different factors which lead to the development of the nine HOT lanes in their respective corridors

- the objectives of the HOT lanes

- changes made in the corridor due to HOT lane implementation

- different impacts of the HOT lanes

- the extent to which the objectives of the HOT lanes were achieved

Using three pairs of HOT lanes with similar design and operational characteristics, comparisons were made to examine the impacts of the similar HOT lanes in two different corridors.

With strict registration requirement for HOV3+ in I-95 there were indications of some carpoolers breaking up in to lower occupancy. Tolled access for HOV2s in I-95 as well as SR 91 resulted in lower usage of the Express Lanes by the HOV2s (fewer than 30 percent of the total corridor HOV2s) as compared to a conventional HOV lane (60 percent) where HOV2 access is free. The effect of availability of transit on the HOT lanes can also be seen from SR 91 and I-95. In SR 91, the Express bus does not use the Express Lanes and there was almost no change in its ridership after the Express Lanes were implemented. However, in I-95, the Express bus uses the Express Lanes and travel time of buses decreased by 17 minutes due to Express Lanes implementation. The Express bus ridership also increased by 30 percent.

In SR167 and I-25 HOT lanes, the exogenous factors like gas prices and economic recession seemed to influence the usage of the HOT lanes. In both the Express Lanes, carpool usage was positively correlated to the gasoline prices while the correlation was even stronger in SR 167. In I-25, the increasing unemployment rate coincided with the decreasing toll paying travelers. In SR 167 there were also indications of mode shifts among the transit, carpool and toll paying SOVs 
due to fluctuating gas prices. With declining gas prices, the transit and carpool usage went down while toll paying users increased.

Also, an inverse relationship between the convenience of access points and the safety perceived by the HOT lane users was found. Express Lanes in I-15, San Diego and I-25, Denver have concrete barrier separation and no intermediate access points. While Express Lane users in both the corridors were satisfied with the safety aspect of the Express lanes, it came at the cost of increased access inconvenience. I-15 Express Lanes in Salt Lake City reduced the access points from unrestricted in previous HOV lanes to limited in Express Lanes. As a result, a more predictable merging led to an increase in the perceived safety of the Express lanes as well as speed of the corridor. On the other hand, some carpoolers mentioned not using the Express Lanes anymore because of access inconvenience. The access inconvenience was also mentioned by previous carpoolers in HOV lanes in I-95 as one of the reasons for not using the Express Lanes. These findings underscore the importance of outreach programs during the planning process of the HOT lanes to minimize the confusion among the previous users of the HOV lanes and spreading awareness among them regarding the increased safety benefits. 


\section{REFERENCES}

95 Express website.<http://www.95express.com/> (accessed on March 2,2010)

ABC Ramps website.<http://www.abc-ramps.com/Default.aspx> (accessed on March 21, 2010).

Brownstone, D. Ghosh, A.Golob, T. F. Kazimi, C. Amelsfort, D.V., 2003. Drivers'

willingness-to-pay to reduce travel time: evidence from the San Diego I-15 congestion pricing project, Transportation Research Part A,373-387.

Buckeye, K.R., and Munnich, L.W., 2004. Value pricing outreach and education: key steps in reaching high occupancy toll lane consensus in Minnesota. Transportation Research Board, $83^{\text {rd }}$ Annual Meeting, Washington, D.C.

Burris, M. W. and Winn, J. R., 2006. Slugging in Houston- casual carpool passenger characteristics, Journal of Public Transportation, 9(5).

Burris, M. and Stockton, B.R., 2004. HOT Lanes in Houston- 6 Years of experience.. Journal of Public Transportation, 7( 3)

Burris, M.W. and Appiah, J., 2004. An Examination of Houston's QuickRide Participants by Frequency of QuickRide Usage, Journal of the Transportation Research Board 1864, TRB, National Research Council, Washington, D.C, 22-30.

Cain, A., 2009 Miami Urban Partnership Agreement (UPA) project, Phase 1A - transit evaluation. prepared by centre for urban transportation research, University of South Florida, Tampa, Florida. Prepared for Federal Transit Administration (FTA).

Cambridge Systematics Inc., 2006a.I-394 MnPASS technical evaluation: final report. Prepared for Minnesota Department of Transportation, Oakland, California. 
Cambridge Systematics,. Inc., 2006 I-95 high-occupancy vehicle lane monitoring report. Prepared for Florida Department of Transportation.

Choueiri, E. M., R. Lamm, J. H. Kloeckner, and T. Mailaender, 1994. Safety aspects of individual design elements and their interactions on two-lane highways. Transportation Research Record: Journal of the Transportation Research Board, No. 1445, Transportation Research Board of the National Academies, Washington, D.C., 34-36

CDOT ,2006. I-25 Express Lanes - policy \& operations, intergovernmental agreement between Colorado tolling enterprise (a unit of CDOT) and Regional Transport District (RTD). Obtained from Stacey Stegman, Colorado DOT.

CDOT ,2007. Summary of I-25 Express Lanes (February 2007)

Chum, G.L. and Burris, M.W. ,2008. Potential mode shift from transit to SOV on a HOT lane, Transportation Research Record: Journal of the Transportation Research Board, No. 2072, Transportation Research Board of the National Academies, Washington, D.C., 10-19.

City of Seattle website http://www.cityofseattle.net/transportation/parking/carpool.htm. Accessed on March 21, 2010.

Congressional Budget Office website http://www.cbo.gov/ftpdocs/97xx/doc9750/AppendixA.8.2.shtml (accessed on February 27, 2010)

Dart, K.O., \& L. Mann., Jr., 1970. Relationship of rural highway geometry to accident rates in Louisiana. In Highway Research Record: Journal of the Transportation Research Board, No. 313, Transportation Research Board of the National Academies, Washington, D.C.,1-15. 
Dahlgren, J., 1999. High occupancy vehicle/toll lanes: how do they operate and where do they make sense? (Working Paper), University of California, Berkeley.

Dahlgren, J., 2002. High-occupancy/toll lanes: where should they be implemented? Transportation Research Part A, 239-255.

DeLuca, F.J., 1985 Effects of lane width reduction on safety and flow: Proceedings of the highway division of the American society of civil engineers. Nashville, Tennessee.

EIA (Energy Information Administration) website < http://www.eia.doe.gov $>$ Accessed February 20, 2010

FDOT, 2009. Final Report- 95 express midyear report project status for urban partnership agreement. Prepared by Florida Department of Transportation, Miami, Florida.

FDOT District Six ,2010 95 Express monthly operations report December 2009.

Ferguson, E., 1997. The rise and fall of American carpool: 1970-1990. Transportation 24, $349-376$

Finance Roundtable. Summary of Questions Asked by the Audience. Accessed online from http://www.hhh.umn.edu/centers/slp/vp/pdf/rethinking_trans_finance_roundtable.pdf

Ghosh, A.,2000. To pay or not to pay: commuters' mode choice decision under congestion pricing, Department of Economics, University of California, Irvine.

Golob, T.F. Golob, J. and Supernak, J., 2001. I-15 congestion pricing project monitoring and evaluation services, task 8, phase II year three attitudinal panel study, San Diego, California.

Golob, T.F., Golob, J., and Supernak, J.,1999. I-15 congestion pricing project monitoring and evaluation services, phase I, overall report, San Diego, California. 
Goldstine, R., 1991 Influence of road width on accidents rates by traffic volumes. In Transportation Research Record: Journal of the Transportation Research Board, No. 1318, Transportation Research Board of the National Academies, Washington, D.C., 1991, pp. 64-69.

Hadi, M.A., J.Aruldhas., L.F. Chow., and J.A Wattleworth, 1995. Estimating safety effects of cross-section design for various highway types using negative binomial regression.

In Transportation Research Record: Journal of the Transportation Research Board, No. 1500, Transportation Research Board of the National Academies, Washington, D.C., 169-177.

iCommute website <http://www.icommutesd.com/Carpool.aspx>(Accessed on March 13, 2010).

Johns, B. Thompson, N. Sallman,D. Zmud, J. Munnich, L. and Sheehan, S., 2006. I-394 MnPASS - a new choice for commuters: how is it working? Rethinking Transportation

Kim, E.J., 2000.HOT Lanes: A comparative evaluation of costs, benefits and performance. Ph.D. dissertation, Urban Planning, University of California, Los Angeles.

Kimley-Horn and Associates, Inc and Cambridge Systematics, Inc., 2008 South Florida Interstate 95 Express project: lessons learned. Prepared for the Florida Department of Transportation. Available online at $<$ http://ops.fhwa.dot.gov/tolling_pricing/value_pricing/pubs_reports/projectreports/sfl_95expr ess_proj/index.htm> Accessed on March 13, 2009.

Li, J., 2001. Explaining high-occupancy-toll lane use, Transportation Research Part D , 61-74.

Mastako, K. A. Rillet, L. R. and Sullivan, E. C.,1998.Commuter behavior on California state route 91 after introducing variable-toll express lanes, Transportation Research Board 1649, TRB, National Research Council, Washington, D.C, 47-54. 
METRO website< http://www.ridemetro.org/SchedulesMaps/HOV/us290.aspx>( accessed on March 3, 2010)

MnDOT, 2004. MnPASS I-394 Express Lane community task force, Final Report.

MnDOT, 2008. I-394 HOV report including 2008- $1^{\text {st }}$ quarter January - March. Prepared by Regional Transport Management Centre.

Munnich, L.W.,2010.Enhancing livability and sustainability by linking congestion pricing with transit. Transportation Research Record: Journal of the Transportation Research Board, No. 2492, Transportation Research Board of the National Academies, Washington, D.C.,129136.

Munnich, L.W. and Buckeye, K. R., 2007. I-394 MnPASS high-occupancy toll lanes planning and operational issues and outcomes (lessons learned in year 1). Transportation Research Record: Journal of the Transportation Research Board, No. 1996, TRB, National Research Council, Washington, D.C., 49-56.

NCHRP Report 197. 1978. Jorgensen and Associates, Inc. Cost and safety effectiveness of highway design elements, NCHRP Report 197, Transportation Research Board.

Bomberg, M. and Kockelman, K.M., 2007. Traveler response to the 2005 gas price spike, presented at the 86th Annual Meeting of the Transportation Research Board.

RTD-Denver website < http://www.rtd-denver.com/HowToPark.shtml> (accessed March 22, 2010)

Parkany, E., 1999. Can high-occupancy/toll lanes encourage carpooling? case study of carpooling behavior on the 91 Express Lanes. Journal of the Transportation Research Board 1682, TRB, National Research Council, Washington, D.C, 46-54. 
Poole, R.W., and Orski, C.K., 1999. Building a case for HOT lanes: a new approach to reducing urban highway congestion. policy study No. 257. Los Angeles: Reason Public Policy Institute.

Poole, R.W., and Orski, C.K., 2000. HOT Lanes: a better way to attack urban highway congestion. 23(1), Regulation, Cato Institute, Washington D.C.

Potts, I.B., D.W. Harwood., and K.R. Richard, 2007. Relationship of lane width to safety on urban and suburban arterials. In Transportation Research Record: Journal of the Transportation Research Board, No. 2023, Transportation Research Board of the National Academies, Washington, D.C., 63-82

Puget Sound trends ,2009< http://www.psrc.org/publications/pubs/trends/index.htm> (accessed on February 15, 2010)

RITA website <http://www.its.dot.gov/JPODOCS/REPTS_TE/13668_files/chapter_7.html> (accessed on February 15, 2010)

Safirova, E. Gillingham, K. Harrington, W. and Nelson, P., 2003. Are HOT lanes a hot deal? the potential consequences of converting HOV to HOT lanes in northern Virginia. Urban complexities issue brief 03-03. Resources for the Future, Washington D.C.

SANDAG ,1999. Report to the California legislature: San Diego's Interstate 15 congestion pricing \& transit development demonstration program as required by Section 149.1(g) of the Street \& Highways code.

SFCS (South Florida Commuter Services), 2009. June 2009 - 95 Express Survey Results. Obtained from Phil Winters, Centre for Urban Transportation Research, University of South Florida, Tampa, Florida. 
Shin, S. and Hickman, M., 1999. Effectiveness of the Katy Freeway HOV-lane pricing project preliminary assessment. Transportation Research Record: Journal of the Transportation Research Board, No. 1099, Transportation Research Board of the National Academies, Washington, D.C., 2010, 97-104.

Smith, L., 2007. Houston HOT Lanes Program Review, Houston METRO.

SR 167 HOT lanes website <www.wsdot.wa.gov/Projects/SR167/HOTLanes> (accessed on January 10, 2010).

Stockton, W.R., Grant, C.L., Hill, C.J., McFarland, F., Edmonson, N.R. and Ogden, M.A., 1998. Feasibility of priority lane pricing on the Katy HOV lane: feasibility assessment. Prepared for TXDOT.

Stone, C., 2009. SR 167 HOT lanes pilot project: first annual performance summary May 2008 - April 2009, Toll Division, Washington State Department of Transportation, Seattle, Washington.

Sullivan E. C. and Harake, J. E., 1998. California route 91 toll lanes impacts and other observations, Journal of the Transportation Research Board 1649, TRB, National Research Council, Washington, D.C, 55-62.

Sullivan, E., 1998. Evaluating the impacts of the SR91 variable toll express lane facility. Final report for State of California Department of Transportation, Sacramento.

Sullivan, E., 2000. Continuation study to evaluate the impacts of the SR-91 value-priced express lanes: Final report, Prepared for California State Department of Transportation, San Luis Obispo, California. Available on-line at (http://ceenve.calpoly.edu/sullivan/SR91/finalrpt/FinalRep2000.pdf).(accessed on December 12, 2002). 
Supernak, J. Golob, J. Golob, T.F. Kaschade, C. Kazimi, C. Schreffler, E. and Steffey, D.,2002. San Diego's Interstate 15 congestion pricing project: traffic-related issues, Transportation Research Record: Journal of the Transportation Research Board, No. 1812, Transportation Research Board of the National Academies, Washington, D.C., 43-51.

TCRP-12, 2000. Traveler response to transportation system changes, Interim Handbook. Prepared for Transit Cooperative Research Program, Transportation Research Board, National Research Council, TCRP Project B-12.

Tone, J., PB Network ,2009.Adding HOT to Denver's I-25 HOV/ BRT Lanes, Denver, Colorado.

TTI ,2004. Houston value pricing pilot program- Quickride non-user survey analysis of comments. Prepared for TxDOT.

Turnbull, K.F., 2008. High occupancy toll lanes and public transportation. Journal of the Transportation Research Board 2065, TRB, National Research Council, Washington, D.C, 3640 .

Turnbull, K.F., 2002.Affects of changing HOV lane occupancy requirements: El Monte busway case study, Prepared by Texas Transportation Institute. Prepared for FHWA.

Turnbull, K.F., 2005. 12th international HOV systems conference: improving mobility and accessibility with managed lanes, pricing, and BRT - conference proceedings. Prepared for FHWA. Accessed from FHWA Office of Operations website $<$ http://ops.fhwa.dot.gov/publications/12hovsysconf/breakout17.htm> (Accessed on February $18,2010)$

\section{Transportation Choices Coalition}

$<$ http://www.transportationchoices.org/oneshotathotlane.asp> (accessed on March 2, 2010) 
Utah DOT website < http://www.udot.utah.gov/expresslanes/ > (accessed on March 23, 2010)

Ungemah, D. Swisher, M. and Tighe, C.D., 2005.Discussing high-occupancy toll lanes with the Denver, Colorado, Public. Transportation Research Record: Journal of the Transportation Research Board, No. 1932, Transportation Research Board of the National Academies, Washington, D.C., 129-136.

Vladisavljevic, I.*, Martin, P.T., Jovanovic, D., Stevanovic, A.,2008. A high-occupancy/toll lane experiment on the I-15 in the Salt Lake City metropolitan region: A Traffic Flow Evaluation. Submitted to the TRB.

Wachs, Martin., 2003. A dozen reasons for raising gasoline taxes. UC Berkeley: Institute of Transportation Studies. Accessed online from http://www.escholarship.org/uc/item/2000f8t0 (accessed on March 1, 2010)

Weinstein, A. and Sciara, G., 2004. Assessing the equity implications of hot lanes. prepared for the Santa Clara Valley Transportation Authority.

Wilbur Smith Associates, 1996. Task 2-B, roadway operations and traffic conditions, baseline travel data and analysis, pre-project baseline data set.

Wilbur Smith Associates, 1997. I-15 congestion pricing project, task 1-A, project concept plan - B, full operations implementation. Prepared for San Diego Association of Governments.

Wilbur Smith Associates, 2002.Volume 1 traffic, revenue and traffic operations: I-15 managed lanes value pricing project planning study. Prepared for San Diego Association of Governments. Available online at (http://www.sandag.org/index.asp?projectid=67\&fuseaction=projects.detail) $($ accessed February 3, 2009). 
WSDOT ,2003. HOT lanes pilot project analysis.

WSDOT ,2005. State Route 167 High Occupancy Toll (HOT) lanes pilot pre-implementation project, Proposed Scope of Work for FHWA Value Pricing Pilot Program. Prepared for FHWA.

WSDOT ,2009. ST 167 HOT Lane Pilot Project, First Annual performance Report (May 2008- April 2009)

WSDOT ,2009a SR 167 HOT Lanes, January 2009 Focus Groups Final Report.

WSDOT ,2010. ST 167 HOT Lane Pilot Project Performance Update- Winter 2010.

Yan, J. Small, K. A. and Sullivan, E.C., 2002. Choice models of route, occupancy, and time of day with value-priced tolls. Journal of the Transportation Research Board 1812, TRB, National Research Council, Washington, D.C, 69-77.

Zmud, J., 2006. I-394 MnPASS project evaluation attitudinal panel survey wave 2: Final report. Available online at (http://www.mnpass.org/systemstudy.html) ( accessed on February 23, 2009).

Zmud, J., 2006a. I-394 MnPASS project evaluation attitudinal panel survey wave 3: Final report. Available online at (http://www.mnpass.org/systemstudy.html) (accessed on February 23, 2009).

Zegeer, C.V., D.W. Reinfurt., J. Hummer., L. Herf., and W. Hunter, 1987. Safety effects of cross-section design for two- lane roads. Publication FHWA-RD-87-008, Federal Highway Administration. 


\section{VITA}

RAHUL GOEL

PERMANENT ADDRESS: C/o Sh. K G Goel, House No. 47, Dwarkapuri, Muzaffarnagar, UP, India, 251001

DEPARTMENT ADDRESS: Department of Civil Engineering, Texas A\&M University 3136 TAMU, College Station, Texas 77843-3136, USA

EMAIL: rahulatiitd@gmail.com

DEGREE: Bachelor's of Technology in Civil Engineering 2008, Indian Institute of

Technology, Delhi, India 